\title{
DISCLAIMER
}

This report was prepared as an account of work sponsored by an agency of the United States Us ancy thereof, nor any of their Government. Neither the United Stales Government nor any ances legal liability or reaponsiemployees, makes any warranty, express or implied, of acs information, apparatue, product, or bility for the sccuracy, compteteness, or usefulness of any information. apposely privately ewned rights. Referprucess disclosed, or represents that its use would not infringe previce by unde name, trademark, ence herein to any spocilic commercial product, process, or service by its endorsement, rocommanufacturer, or otherwise does not necessarily constivte or inily its endorsement The views mendation. or favoring by the United States Governnient or sny agency thenest these of the and opinions of authors expressed herein do not

United States Government or any asency thereof

\section{Kinetic Modelling of Krypton Fluoride Laser Systems}

\author{
Kenneth S. Jancaitis \\ (Ph.D. Thesis)
}

Manuscript date: November 1, 1983

\section{LAWRENCE LIVERMORE NATIONAL LABORATORY University of California - Livermore, California - 94550}


Kinetic Modelling of Krypton Fluoride Laser Systens

By

Kenneth Stephen Jancaitis

B.S. (California Institute of Technology) 1975

\section{DISSERTATION}

Submitted in partial satisfaction of the requirements for the degree of DOCTOR OF. PHILOSOPHY

in

Physics

in the

GRADUATE DIVISION

OF THE

UNIVERSITY OF CALIFORNIA, BERKELEY

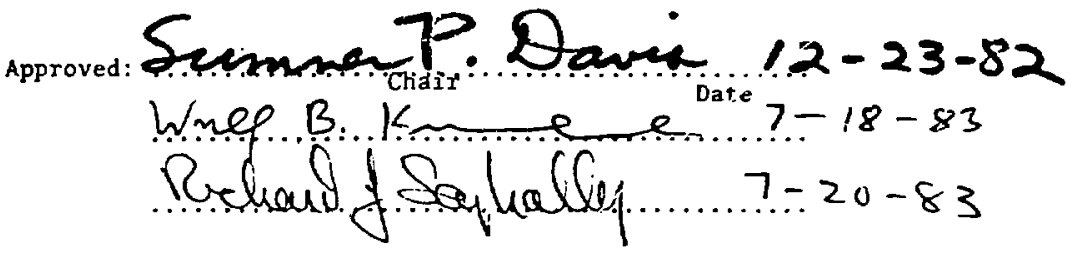


Kinetic Modelling of Krypton Fluoride Laser Syszems

\author{
Kenneth S. Jancaitis
}

\title{
Abstract
}

A kinetic model has been developed for the $\mathrm{KrF}^{\star}$ rare gas halide laser system, specifically for electron-beam pumped mixtures of krypton, fluorine, and either helium or argon. The excitation produced in the laser gas by the e-beam was calculated numerically using an algorithm checked by comparing the predicted ionization yields in the pure rare gases with their experimental values. The excitation of the laser media by multi-kilovolt $X$-rays was also modeled and shown to be similar to that produced by high energy electrons. A system of equations describing the transfer of the initial gas excitation into the laser upper level was assembled using reaction rate constants from both experiment and theory. A one-dimensional treatment of the interaction of the laser radiation with the gas was formulated which considered spontancous and stimulated emission and absorption. The predictions of this model were in good agreement with the fluorescence signals and gain and absorption measured experimentally. 
To gather input for this model, rare gas halide dimer ano trimer fluorescence signals were studied using $50 \mathrm{~ns} 600 \mathrm{keV}$ electron-beam excitation of binary mixtures of Ar or $\mathrm{Kr}$ combined with either $\mathrm{F}_{2}$, $\mathrm{NF}_{3}$, or $\mathrm{SF}_{6}$, and buffered mixtures containing $\mathrm{Kr}, \mathrm{F}_{2}$, and either He, Ne, or Ar. A simple model was developed to analyze the data at both high and low $\left(\leqslant 50 \mathrm{~A} / \mathrm{cm}^{2}\right)$ current densities, yielding estimates for the trimer radiative lifetimes and collisional quenching coefficients and the rate constants for the formation of the trimers.

The apparatus used to study rare gas nalide fluorescence was converted into a laser cavity by placing mirrors inside the gas cell. With either helium or argon as the buffer gas the krypton and fiur, ine pressures yielding the maximula laser efficiency were determined for two values of the current density $\left(160\right.$ and $\left.430 \mathrm{~A} / \mathrm{cm}^{2}\right)$. The predictions of the laser kinetics model for these systems were compared to the experimental observations. Within the limits of the one-dimensional model good agreement was obtained over the range of parameters typical of laser operation. 


\section{Acknowledgements}

With pleasure I ackno'sledge the help and encouragement given me throughout this work by my major advisor, Professor Sumner P. Davis. I am especially grateful to $\mathrm{Dr}$. Howard T. Powell of the Lawrence Livermore National I zuoratory for his suggestions and aid concerning the experimental portions of this thesis. I have also benefited by discussions with numerous mernbers of the Advanced Laser Research and Development Group at LLNL.

During this work I was supported for five years by a fe? lowship from the Fannie and John Hertz Foundation. This research was supported under the auspices of the U. S. Department of Energy by the Lawrence Livermore National Laboratory under contract W-7405-ENG-48. 
Table of Contents

I. INTRODUCTION

A. Historical Perspective 1

8. Physical Considerations 4

C. Outline 10

$\begin{array}{ll}\text { Figures } & 14\end{array}$

$\begin{array}{ll}\text { References } & 16\end{array}$

II. THE EXCITATION OF KRYPTON FLUORIDE LASER

MEOIA BY HIGH ENERGY ELECTRONS 18

A. The Fowler Equation 18

B. Electron Collisional Excitation and Ionization Cross Sections 25

C. The Numerical Solution of the Fowler $\begin{array}{ll}\text { Equation } & 27\end{array}$

D. Discussion 31

Tables 34

Figures $\quad 40$

References $\quad 46$

III. THE I:XCITATION OF KRYPTON FLUORIDE LASER

MEDIA BY KILOVOLT X-RAYS 48

A. Introduction 48

B. Physical Processes 50

C. Numerical Solution of the $X-r a y$ Deposition Problem 


\section{THE EXIITATION OF KRYPTON FLUORIDE LASER}

MEDIA BY KILOVOLT $X$-RAYS (cont.)

Tables

Figures

References

1V. KRYPTON FLUORIOE LASER KINETICS 65

A. Introduction 65

B. Particle Kinetics 66

C. Interaction of the Radiation Field with the Laser Medium 71

(i) Spontaneous and Stimulated Emission 71

(ii) Absorption 74

D. Numerical Methods $\quad 75$

Tables $\quad 85$

$\begin{array}{ll}\text { Figures } & 100\end{array}$

$\begin{array}{ll}\text { References } & 109\end{array}$

V. THE FORMATION AND QUENCHIYG OF KrF* AND $\mathrm{Kr}_{2} \mathrm{~F}^{*} \quad 115$

A. Introduction $\quad 115$

B. Buffered $\mathrm{Kr}-\mathrm{F}_{2}$ Mixtures $\quad 117$

C. Discussion and Conclusions 123

$\begin{array}{ll}\text { Figures } & 125\end{array}$

$\begin{array}{ll}\text { Appendix } & 131\end{array}$

I. Introduction 132

II. Experimental Setup 134

TII.Experimental Results 139 
THE FORMATION AND QUENCHING OF $\mathrm{KrF}^{*}$ AND $\mathrm{kr}_{2} \mathrm{~F}^{*}$ (cont.) Appendix (cont.)

IV. Analysis

v. Results and Interpretation 147

VI. Conclusions 154

Appendix 156

Figure Captions $\quad 160$

Figures 163

$\begin{array}{ll}\text { References } & 176\end{array}$

VI. THE OBSERVATION AND MODELLING OF KrF LASER ACTION 178

A. Introduction 178

B. Experimental Technique 180

C. Laser Model 184

D. Results of Experiment and Calculation 190

(i) Introduction 190

(ii) Argon Burfered Mixtures 192

(iii)Helium Buffered Mixtures 195

E. Discussion 198

Tables 203

$\begin{array}{ll}\text { Figures } & 205\end{array}$

$\begin{array}{ll}\text { References } & 218\end{array}$

MICROFICHE LISTINGS OF COMPUTER PROGRAMS 219 
Chapter I

Introduction

\section{A. Historical Perspective}

The $\mathrm{KrF}^{*}$ rare gas halide (RGH) laser is a member of a larger class of systems known as excimer lasers, which may be loosely defined as those lasers which operate on electronic molecular transitions having a repulsive lawer state potential. Before we begin the detailed discussion of the physical principles which are relevant to excimer laser perfomance in general, and $\mathrm{KrF}$ * laser performance in particular, it will prove useful to prese-t a brief outline of the events which have led to the current level of development and understanding of these systems. This will provide a backdrop to the understanding of both the physical problems involved and the place this thesis is meant to fill in the literature.

The history of excimer iasers may be said to have begun with the identification of the bound-free continuum radiations from the rare gas dimers and the development of lamps for their efficient production by Tanaka and Zelikoff in the 1950's. ${ }^{1-3}$ The first suggestion that such transitions might be used to provide gain was made by Houtermens 
in $1960,{ }^{4}$ and is illustrated in Figure 1. Atoms which in their ground state interact along a repulsive potential are excited via a non-thermal mechanism to a level where their interaction is attractive, upon which they form a bound state. Provided there are no potential curve crossings from this bound complex to the ground level, it will decay primarily via radiation (if the transition is optically allowed). In this case, when the ground state curve is sufficiently repulsive (i.e. when $\Delta E \gg k T_{g}$, where $\Delta E$ is the energy of the decay product relative to the ground state atoms at infinite separation and $T_{g}$ is the gas temperature) the radiative decay product will be rapidly dissociated and any problems with bottlenecking in the lower state eliminated.

The first efforts toward the development of an excimer laser sought to obtain gain using the $\mathrm{H}_{2}\left(a^{3} \Sigma_{g}-b^{3} \Sigma_{u}\right)$ transition. 4 The analys is of $\mathrm{Pa} / \mathrm{mer}{ }^{5}$ however indicated that competing processes would lead to absorption at the relevant wavelengths; later experimental work examining the $490 \mathrm{~nm}$. fluorescence continuum in $\mathrm{Hg}$ also showed loss rather than gain. ${ }^{6}$ In 1971 Basov and coworkers finally constructed the first working excimer laser, obtaining gain at $171 \mathrm{~nm}$. on the $\mathrm{Xe}_{2}{ }^{*}$ continuum using liquid $x e$ pumped with an electron beam. ${ }^{7}$ Gas phase electron beam pumped rare gas dimer lasers were soon developed on the $\mathrm{Ar}_{2}{ }^{\star}, \mathrm{Kr}_{2}{ }^{\star}$ and $\mathrm{Xe}_{2}{ }^{*}$ excimer transitions, although the observed gains were much less than initially predicted from the fluorescence efficiencies measured under similar conditions. $8-10$ 
The concept of the excimer laser was extended to non-homonuclear systems in 1974 when lasing from the rare gas oxides $\mathrm{ArO}^{\star}, \mathrm{KrO}^{*}$, and $\mathrm{Xe} 0$ * between malecular levels corresponding to the oxygen ${ }^{\mathrm{T}} \mathrm{S}_{0}-{ }^{1} \mathrm{D}_{2}$ auroral transition was denonstrated by Powell et. al. ${ }^{11}$ An electron beam was again used to obtain energetic pumping of the laser medium. Gain was observed only after the excitation of the gas by the e-beam had ceased, while during the current pulse the medium acted as an absorber at the laser wavelength.

Bound-free emission from the rare gas halide dimers was first observed in the identification of the $\mathrm{ArCl}$ * continuum by Golde and Thrush in 1974. ${ }^{12}$ Fluorescence from other RGH dimers (XeF*, XeCl*, etc.) was soon reported. and the $\mathrm{KrF}^{+} \mathrm{B}-\mathrm{X}$ emission observed and discussed by Brau an' rwing in 1975. ${ }^{13}$ Gain in RGH systeris was ostained in the same year by both Searles and Hart (KeBr*) ${ }^{14}$ and Brad and Ewing $\left(X_{e F} F^{1}{ }^{15}\right.$ This pumping thresholds for the RGH lasers were found to be significantly less that that for the previously demonstrated excimer lasers. The most promising of these systems was the K.F* laser, for which medium efficiencies as high as $15 \%$ were reported. 16,17

Because of the volume scaleability of the electron beam pumped RGH lasers, interest has been generated in their probable use as drivers for inertial confinement fusion systems. ${ }^{18,19}$ To this end, high power $\mathrm{KrF}^{*}$ lasers are currently under development at several laboratories. 20-21 Such systems have been designed to produce small- 
signal gains on the order of $e^{10}-e^{15}$, with output energies of around 1 kilojoule in a pulse lasting on the order of 100 nanoseconds.

\section{B. Physical Considerations}

The de:elopment of excimer lasers as related in the previous section was hirdered by the discovery of initially unexpected media absorptions and the relatively high pumping rates $\left(\geqslant 1 \mathrm{MW} / \mathrm{cm}^{3}\right.$ ) required to obtaill gain. In this section we will discuss the physical properties of excimer lasers which result in these limitations. At the same time we will introduce terms and concepts which will be referred to in the fo?lowing chapters.

We begin with a simple analysis of the excimer laser transition illustrated in Figure 1 . The stimulated emission cross section as a function of frequency is given by

$$
\sigma_{s}(\nu)=\frac{\lambda^{2}}{8 \pi} \frac{1}{T_{r}} g(\nu)
$$

where $\lambda=c / \nu, \tau_{r}$ is the radiative decay lifetime of the upper state, and $g(\nu)$ is the normalized lineshape factor $\left(\int_{g}(\nu) d \nu=1\right){ }^{22}$ at the laser wavelength, $g(\nu)$ is given approximately by $\Delta \nu^{-1}$, where $\Delta \nu$ is the width of the spontaneous emission spectrum. The magnitude of this 
linewidth may be estimated according to the Frank Condon principle $e^{23}$ as

$$
h \Delta \nu=-V_{L}^{\prime}\left(R_{0}\right) \Delta R_{U}
$$

where $V_{L}^{\prime}\left(R_{0}\right)$ is the radial derivative of the lower state potential evaluated at the equilibri,m separation of the bound upper level and $\Delta R_{U}$ is the spatial extent cf the upper state in its potential well.

We see from Eq. 2 that the width of the excimer emission continuum is direcily proportional to the repulsive nature of the lower state potential, as measured by the force that the radiative decay product feels toward dissociation. Coupling this result with Eq. 1 we find that the steeply repulsive nature of the excimer lower state potential curves results in small values for the stimulated emission cross section (typically $10^{-18}-10^{-16} \mathrm{~cm}^{2}$ ), as compared with the values for bound-bound molecular $\left(10^{-17}-10^{-14} \mathrm{~cm}^{2}\right)$ and a lowed atomic transitions $\left(10^{-14}-10^{-11} \mathrm{~cm}^{2}\right)$.

It is tie low value of the stimulated emission cross section which mandates the high pumping powers required to obtain significant gain in excimer lasers, as can be shown via a simple analysis. In a steady-state system $g_{0}$, the small-signal gain coefficient in the absence of medium absorptions is given by

$$
g_{0}=\sigma_{\mathrm{s}} f N^{*}
$$


where $N^{*}$ is the excited state density and $f$ is the fraction of the upper state molecules available to the laser transition (e.g. in KrF* the excited state density is believed to be divided equally between two levels [the $B$ and $C$ stakes], so for the $B-X$ laser transition $f=.5)$. The pumping power required to achieve the excited state density $N^{*}$ is given by

$$
P_{D}=\frac{N^{*}}{T_{U}} \frac{E^{\prime}}{\eta_{B}}
$$

where $r_{u}$ is the effective lifetime of the upper state, $E^{\prime}$ is the average energy of the initial excitation produced in the laser medium by the pumping mechanism, and $\eta_{B}$ is the branching ratio from the initial excitations to the laser upper state. Combining Eqs. 3 and 4 yields an estimate for the pumping power required to achieve a given gain:

$$
\left.P_{D}=, E^{\prime} / \tau_{u} \eta_{B} f \sigma_{S}\right) g_{O}
$$

Inserting parameters corresponding to the $\mathrm{KrF}^{*}$ system $\left\langle\mathrm{E}^{\prime} \approx 15 \mathrm{eV}\right.$, $\tau_{u} \approx 5 \mathrm{~ns} ., \eta_{B} \approx 1, \sigma_{s} \approx 2.5 \times 10^{-16} \mathrm{~cm}^{2}$ ), we find that even ignoring any losses pumping on the order of $.5 \mathrm{MW} / \mathrm{cm}^{3}$ would be neccessary to reach a gain of $10 \% \mathrm{~cm}^{-1}$. Similar calculations for the rare gas excimers and the rare gas oxides indicate that they would require on the order of 5 and $50 \mathrm{MW} / \mathrm{cm}^{3}$, respectively to achieve a similar value for $g_{0}$.

The next point which must be noted is that the simple pumping 
scheme implied by figure 1 is not realized in actual excimer lasers. The energy deposited in the laser medium by the excitation mechanism generally passes through a number of intermediate states before it is transiered to the upper state of the laser transition. Taking the formation of $\mathrm{KrF}^{\star}$ as an example, atomic ions (e.g. $\mathrm{kr}^{+}, \mathrm{F}^{-}$), excited atoms (e.g. $\left.k r^{\star}, k r^{\star *}\right)$, dimer ions $\left(e . g . k r_{2}^{+}\right)$, and rare gas excimers (e.g. $\mathrm{Kr}_{2}{ }^{*}$ ) are just some of the precursors to the formation of the $\mathrm{KrF}^{*}$ dimer. The gas excitation is exchanged between these species through a series of two- and three-body chemical reactions, which require a gas pressure $\geqslant 1 \mathrm{~atm}$. (which is in general provided by the addition of a lower $-Z$ rare gas buffer) for the efficient formation of the lasing state.

We see by the arguments above that the operation of excimer lasers requires a pumping mechanism which can deliver $\geqslant 1 \mathrm{MW} / \mathrm{cm}^{3}$ into a yas medium at pressures $\geqslant 1$ atrin. Historically, this was achieved with the development of high current density ( $\geqslant 100 \mathrm{Amp} / \mathrm{cm}^{2}$ ) eiectron bean sources, as was indicated in Section $A$. It is only for the RGH excimer lasers with their lower pumping thresholds that discharge pumping was subsequently found to be sufficient, 24,25 albeit with lower medium efficiencies than demonstrated for e-beam pumped systems.

The simple picture preserted in Figure 1 also fails in that it does not take into account ary possible transitions leading to absorption at the laser wavelength. In reality, the chemica! rature of the upper level formation introduces into the laser medium a number of states for which absorption of the laser photon via a 
bound-continuum process is energetically possible (e.g. for the KrF* system we have $\mathrm{F}^{-}, \mathrm{Kr}_{2}^{+}, \mathrm{Kr}_{2}{ }^{*}$, etc.). Even the proposed laser upper state itself may prove to be a significant absorber, as has been suggested by Werner et. al. for the $x e_{2}{ }^{*}$ excimer laser. ${ }^{26}$ Finally, those excimers which are not destroyed by radiative means may pruduce throligh further chemical reactions species which also absorb at the laser wavelength.

Absorption in the laser medium has an additiona? infiuence on the performance of excimer lasers beyond the reduction of the net gain, In steady-state the gain coefficient for a driven laser in the absence of absorption is given by

$$
g^{\prime}=\frac{g_{0}}{1+1 / \bar{I}}
$$

where $I$ is the local laser intensity and $g_{0}$ and $I_{s}$ are defined by

$$
g_{0}=\sigma_{s} f N^{*} ; I_{s}=h \nu / f \sigma_{s} \tau_{u}
$$

where $N^{*}$ and $\tau_{u}$ are the laser upper state density and lifetime, respectively, in the limit of vanishingly snlall linser intensity. When absorption is taken into account, the net gain coeficient becomes

$$
g=\frac{g_{0}-\gamma_{0}}{T+I_{S}}-\gamma_{n}
$$

where $\gamma$. is that portion of the total absorption coefficient steming 
from either the lasing state itself or its "descender s", defined as the saturable absorption; and $\gamma_{n}$ is the non-saturable absorption caused by the precursors to the formation of the laser upper level.

The division of the loss into its saturable and non-saturable components has two consequences. The first is that the non-saturable loss limits the maximum intensity which can be passed through the laser to the value:

$$
I_{\max }=I_{S}\left[\frac{g_{0}-\gamma_{0}}{\gamma_{n}}-1\right] \text {. }
$$

Secondly, it affects the local extraction efficiency $\theta$, defined as the ratio of the rate of the removal of the upper states by the lase field to their production rate

$$
\theta=\frac{\left.I \mid \frac{g_{0}-\gamma_{0}}{1+\mathrm{I} / \mathrm{I}_{s}}-\gamma_{n}\right\}}{\left(N^{\star} / \tau_{u}\right) h \nu}=\frac{\frac{I}{I_{s}}\left|\frac{g_{0}-\gamma_{0}}{1+I / I_{s}}-\gamma_{n}\right|}{g_{0}} .
$$

Optimizing $\theta$ as a function of the laser intensity, we find that the maximum extraction efficiency is given by

$$
\boldsymbol{\theta}_{\max }=\left[\sqrt{1-\frac{\gamma_{0}}{g_{0}}}-\sqrt{\frac{\gamma_{n}}{g_{0}}}\right]^{2} .
$$

In Figure 2 we show the dependence of $\theta_{\max }$ on $\gamma_{\text {tot }} / g_{0}$ (where $\gamma_{\text {tot }}=\gamma_{0}+\gamma_{n}$ is the total absorption coefficient) for several values of $\gamma_{n} / \gamma_{\text {tot }}$. The importance of determining the sources af the medium absorption to the consiruction of efficient high power excimer lasers 
is indicated by examining the case $\gamma_{\text {tot }} / g_{0}=.1$. For the situation where the losses are totally saturable we have $\theta_{\max }=90 \%$; while for a scenario where the non-saturable absorption dominates, the maximum medium extraction efficiency is on $1 y 47 \%$.

\section{Cutline}

The purpose of this thesis, as indicated by its title, is to develop a kinetic model for the $\mathrm{KrF}^{*}$ rare gas halide iaser system. Specifically, we have chosen to analyze the behavior of electron beam pumped $\mathrm{Kr}-\mathrm{F}_{2}$ mixtures, buffered with either helium or argon. High energy ( $\geqslant 1 \mathrm{keV}$ ) electron beams as opposed to gas discharges were selected as the excitation mechanism of interest both because of the scaleability of e-beam pumped systems to high power and gain, and because of the simpler electron kinetics which applies in the former case. Helium and argon were picked as the buffer gases because they represent the extremes available, neon being an intermediate case displaying some of the behavior of each of the other two choices.

We begin the analysis in Chapter II with a discussion of the excitation of the laser media by high energy electrons. A techrique known as the Fowler equation ${ }^{27}$ is described which determines the distribution of the energy deposited by the e-beam over the various levels available to the gas atoms once a specification of the relevant energy levels is given along with the electron collisional cross sections for their formation from the ground state. Such a 
specification is determined for each of the rare gases from helium through xenon, and the yields of the excited states in the pure rare gases resulting from e-beam pumping is calculated. The inverse ionization efficiencies (defined as the mean energy deposited per ion pair created) determined in this manner for the pure rare gases compare well with those measured experimentally. Finally, the excitation in two cases representative of krypton fluoride laser gas mixtures is calculated and the qualitative behavior of the variation of the excitation yields with gas mixture is discussed.

In Chapter III we digress slightly by developing a model for the calculation of the excitation of $\mathrm{KrF}^{*}$ laser media by multi-kilovolt $x$-rays. We begin by describing how proposed second generation inertial confinement fusion systems may provide $X$-ray fluxes sufficient to pump high energy RGH lasers. This is followed by an analysis of the physical processes which dominate the transfer of the energy from the $x$-ray flux to the various gas excited levels. The chapter is concluded with a calculation of the excitation yields coming from kilovolt X-ray pumping of the representative KrF* laser mixtures described in Chapter II, along with a comparison of the results to those obtained for e-beam pumping. This comparison indicates that the excitation caused by high energy photons is very similar to that produced by high energy electrons, although the difference in the magnitudes of the X.ray and electron interaction cross sections means that the pumping in the former case generally takes place over much larger volumes. 
In Chapter IV we treat the transfer of the primary excitation produced in the laser medium by the pumping mechanism into the laser upper state, and from thence into the laser output spectrum. The chemical nature of the krypton fluoride laser system requires that this transfer take place via a series of two- and three-body reactions in the laser gas. We therefore begin with an identification of the atomic and molecular species and the reaction processes relevant to the formation and destruction of the $\mathrm{KrF}^{*}$ dimer, along with an estimation of the rates at which these reactions proieed. We then continue with a discussion of the interaction between the laser radiation field and the laser gas, taking into account stimulated and spontaneous emission, along with medium absorption. All this information is combined in a one-dimensional model which integrates the iaser kinetic equations taking into account the spatial, as well as temporal variations of the variolis quantities. Finally, the results from this model are compared with fluorescence signals and gain and absorption measurements determined from experiment.

In Chapter $V$ we present the experimental work performed by the present author and Dr. H. T. Powell on the kinetics of the rare yas halide (RGH) dimer - trimer system. RGH dimer and trimer fluorescence signals were measured from e-beam excited mixtures of either $\mathrm{Ar}$ or $\mathrm{Kr}$ combined with either $F_{2}, N_{3}$, or $S F_{6}$, and al so from buffered mixt.ures containing $\mathrm{Kr}$ and $\mathrm{F}_{2}$ and either $\mathrm{He}$, Ne, or Ar. A simple model is developed to analyze this daia at both high and low ( $\leqslant 50 \mathrm{Amp} / \mathrm{cm}^{2}$ ) current densities. At low currerit densities this analysis results in 
estimates for the RGH trimer radiative lifetimes, the rate coefficients for their destruction in collisions with heavy particles, and the rate constants for their formation from the corresponding RGH dimers. At nigher current densities we find that electron quenching becomes the dominant factor in the trimer decay rate, although we have found no model which accurately predicts the magnitude of its effect.

The laser performance of electron beam pumped mixtures of $\mathrm{Kr}$, $F_{2}$, and either He or Ar is studied in Chapter VI. The experimental setup described in Chapter $V$ was converted into a laser cavity by the addition of U. V. laser mirrors into the gas cell. With either argon or helium as the buffer gas, the krypton and fluorine pressures yielding the maximum laser efficiency were determined for two values of the average current density $\left(160\right.$ and $\left.430 \mathrm{~A} / \mathrm{cm}^{2}\right)$. The laser kinetics mode $\mathbf{i}$ was then used to predict the output performance of the gas mixtures and laser configurations investigated experimentally. Finally, the successes and shortcomings of the present state of the model are discussed, and suggestions made for possible future improvement. 


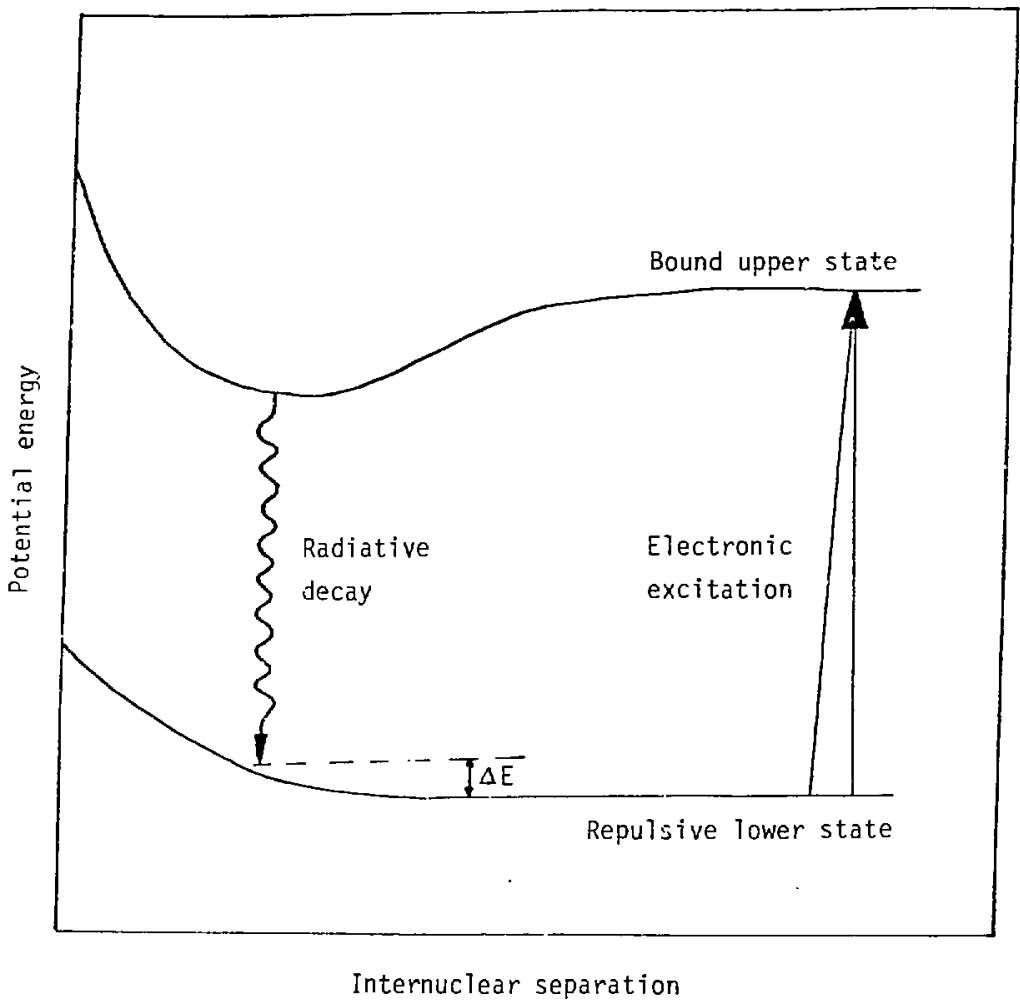

Fig. 1. Potential energy diagram illustrationg the scheme of excimer laser excitation and decay. 


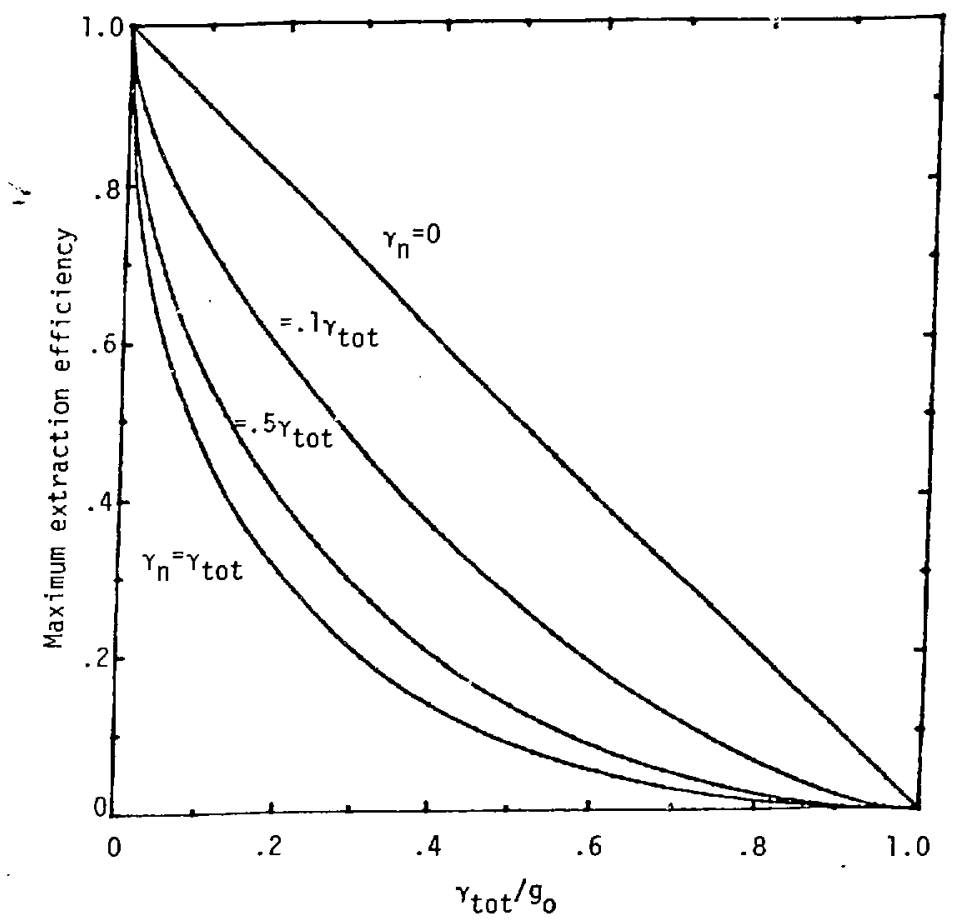

Fig. 2. Maximum laser extraction efficiency vs loss to gain ratio plotted for several values of the relative nonsaturable Toss coefficient. 
References - Chapter I

1. Y. Tanaka and M. Zelikoff, Phys. Rev. 93, 933 (1954)

2. Y. Tanaka and M. Zelikoff, Jrnl. Opt. Soc. Amer. 14, 254 (1954)

3. Y. Tanaka, Jrn1. Opt. Soc. Amer. 45,710 (1955)

4. F. G. Houtermens, Helv. Phys. Acta 33, 933 (1960)

5. A. J. Palmer, Jrnl. Appl. Phys. 41, 438 (1970)

6. R. M. Hill, D. J. Eckstrom, D. C. Lorents, and H. H. Nakano, Appl. Phys. Lett. 23,373 (1973)

7. N. G. Basov, V. A. Danilychev, and Y. M. Popov, Sov. Jrn1. Quant. Elect. 1, 18 (1971)

8. W. M. Hughes, J. Shannon, and R. Hunter, App 1. Phys. Lett. 24, $488(1974)$

9. P. W. Hoff, J. C. Swingle, and C. K. Rhodes, Appl. Phys, Lett. $\underline{23}, 245(1973$

10. J. B. Gerado and A. W. Johnson, IEEE JrnT. Quant. Elect. 9, 748 (1973)

11. H. T. Powel1, J. R. Murray and C. K. Rhodes, Appl. Phys. Lett. $25,730(1974)$

12. M. F. Golde and B. A. Thrush, Chem. Phys. Lett. 29, 485 (1974)

13. C. A. Brau and J. J. Ewing, Jrnl. Chem. Phys. 63, 4640 (1975)

14. S. K. Searles and G. A. Hart, Appl. Phys. Lett. 27, 243 (1975)

15. C. A. Brau and J. J. Ewing, App1. Phys. Lett. 27, 435 (1975)

16. C. A. Brau and J. J. Ewing, Electranic Iransition Lasers, J. I. Steinfeld, ed. (MIT Press, Cambridge, 1976) 
17. M. L. Bhaumik, R. S. Bradford, and E. K. Ault, Appl. Phys. Lett. $\underline{28}, 23(1976)$

18. Laser Program Annnual Report - 1977, UCRL 50021-76, Lawrence Livermore Laboratory (1976) p. 6-2

19. C. M. Stickley, Physics Today 31, 50 (1978)

20. F. O'Neill, Rutherford Laboratory, private communication (1980)

21. Laser Program Annnual Report - 1980, UCRL 50021-80, Laurence Livermore National Laboratery (1980) p. 8-17

22. F. H. Mies, Molec. Phys. 26, 12:3 (1973)

23. J. B. Hasted, Physics of Atomic Collisioris (American Elsevier, New York, 1972) p. 72

24. J. A. Mangano and J. H. Jacob, Appl. Phys. Lett. 27, 495 (1975)

25. R. C. Sze and P. B. Scott, Appl. Phys. Lett. 32, 479 (1978)

26. C. W. Werner, E. V. George, P. W. Hoff, a.d C. K. Rhodes, Appl. Phys. Lett. 25, 235 (1974)

28. R. H. Fowler, Proc. Camb. Philos. Soc. 21, 531 (1923). 
Chapter II

The Excitation of Krypton Flouride Laser Media by High Energy Electrons

\section{A. The Fowler Equation}

The determination of the effectiven: $=$ of a high energy electron in pumping the krypton fluoride laser transition must include not only a specification of the amount of energy deposited in the gas by the electron, but also the detailed partitioning of this energy over the various levels of excitation available to the gas atoms. The first attempt at a solution to a problem of this type was made by $R$. H. Fowler in 1923, who sought to determine the total ionization produced by the passage of an alpha particle through a gas. ${ }^{1}$ In recent years, Fowler's treatment has been extended to the calculation of the yield of all gas excitations produced during the stopping of high energy electrons. Other workers have developed alternate approaches to the solution of this same problem; among them Monte Carlo approximations, ${ }^{2}$ the Spencer-Fano degradation spectrum technique, ${ }^{3}$ and the continuous slowing down approximation. 4

Garvey and Green have compared several of these methods using a common set of cross sections for the excitation of $\mathrm{H}_{2} \cdot{ }^{5}$ They 
found that provided sufficient care was taken in the application of the various approximations all approaches yielded the same solution for the partitioning of the deposited energy. In addition, Inokuti et. al. have shown that the degradation spectrum of the Spencer-Fano equation is the universal adjoint to the sulutions of the Fowler equation for the excitation yields. ${ }^{6}$ The method of solution is thus seen to be primarily a matter of individual preference. We have chosen the Fowler equation for reasons to be described below.

The Fowler equation approach calculates $J_{k}(E)$, the average yield of excitations of state $k$ produced by the stopping of an electron of energy $E$. We have (Equation 1)

$$
J_{k}(E)=p_{k}(E)+\sum_{j} p_{j}(E) J_{k}\left(E-E_{j}\right)+\sum_{i} \int_{0}^{\left(E-E_{j}\right) / 2} p_{j}(E, T)\left[J_{k}\left(E-E_{j}-T\right)+J_{k}(T)\right] d T
$$

In this equation the subscripts $j$ label the excited states having excitation energies $E_{j}$ and the subscripts $i$ label the ionic states with ionization energies $E_{i}$; the label $k$ may refer to either an ionic or excited state. The "p's" are defined as follows: if $\sigma_{j}(E)\left(\sigma_{j}(E)\right)$ is the cross section for electron collisional excitation of the excited (ionic) state $j$ (i) by an electron of energy $E$, and $n_{j}\left(n_{j}\right)$ is the number density of ground state atoms or molecules which may produce state $j(i)$ upon this collision we have

$$
p_{k}(E)=n_{k} \sigma_{k}(E) /\left[\sum_{j} n_{j} \sigma_{j}+\sum_{i} n_{i} \sigma_{i}\right]
$$


where the label $k$ again may represent either an excited or ionic state. Similarly, if $\sigma_{i}(E, T)$ is the cross section for the production of an ionic state $i$ along with a secondary electron of energy $T$ upon collision of an electron of energy $E$ with the ground state species having density $n_{i}$, then

$$
p_{i}(E, T)=n_{i} \sigma_{i}(E, T) /\left[\sum_{j} n_{j} \sigma_{j}+\sum_{i} n_{i} \sigma_{i}\right]
$$

The quantities $p_{j}(E)$ and $p_{j}(E, T)$ are related by

$$
p_{i}(E)=\int_{0}^{\left(E-E_{j}\right) / 2} p_{j}(E, T) d T
$$

where the upper bound on the integral is a consequence of the convention that the electron of lower energy after the ionization is defined as the secondary electron.

Using Eq. 2 one can easily show that

$$
\sum_{j} p_{j}(E)+\sum_{i} p_{j}(E)=1
$$

Thus, in physical terms $p_{j}(E)$ is the probability that an electron of energy $E$ will lose energy by producing the excited state $j$. A similar interpretation applies for both $p_{j}(E)$ and $p_{j}(E, T)$.

With this definition of the "p's" the meaning of each of the terms 
on tiie right hand side of Eq. I becomes readily apparent. The first term is the number of excitations of state $k$ that the primary electron produces directly at energy $E$. : The quantity $p_{j}(E) J_{k}\left(E-E_{j}\right)$ is the product of the probability that the electron will directly create an excited state $j$ times the number of excitations of state $k$ that it would subsequently produce in slowing down in the gas; the second term on the right of Eq. 1 sums these contributions to $J_{k}(E)$ over all exrited states $j$. The integral over the secondary electron energy determines the contribution to $J_{k}(E)$ as a result of jonization collisions; the subsequent slow-down of both the primary electron and the secondaries which it produces have to be considered in this case.

The Fowler equation is solved for $\mathrm{J}_{k}(E)$ upon noting that the energies at which $J_{k}(E)$ must be eviluated on the right hand side of Eq. l are all lower than the energy of interest on the left $h$ and side. Using the initial condition that $J_{k}(E)$ is equal to zero for all $E<E_{k}$, Eq. 1 may then be iterated upward to an arbitrarily high primary electron energy.

Before proceeding with a description of the solution of the Fowler equation for the particular problem of interest, it will be useful to derive some general results which make this approach particularly attractive. It is a well known fact that for sufficiently high energy $E$, the ionization efficiency defined as $\theta_{i}=J_{j}(E) / E$ aproaches a constant value, equal to the inverse of $w_{j}$, the mean energy loss per ion pair created. Inokuti has shown that this behavior can be derived theoretically starting with the Fowler equation. ${ }^{7}$ In what follows 
we extend his treatment to a description of the asymptotic behavior of the excitation efficiency $\theta_{k}=J_{k}(E) / E$ for any state $k$, whether ionic or excited.

Following Inohuti's treatment we postulate that $J_{k}(E)$ may be well approximated (at least at high energies) as

$$
u_{k}(E)=\left(E-U_{k}\right) / W_{k}
$$

Inserting this approximation into Eq. 1, and using Eq. 4 rasutts in

$$
\left(E-U_{k}\right) / W_{k}=p_{k}(E)+\sum_{j} p_{j}(E)\left(E-E_{j}-U_{k}\right) / W_{k}+\sum_{i} p_{j}(E)\left(E-E_{j}-2 U_{k}\right) / W_{k}
$$

Using Eq. 5, this may be manipulated to yieid

$$
W_{k}=\sum_{j} E_{j} p_{j}(E) / P_{k}(E)+\sum_{i} E_{j} p_{i}(E) / p_{k}(E)+U_{k} \sum_{i} p_{i}(E) / p_{k}(E)
$$

Although $W_{k}$ is not strictly a constant, the energy dependence of the various terms given by $p_{j}(E) / p_{k}(E)$ and $p_{j}(E) / p_{k}(E)$ becomes weak for sufficiently large electron energy $E(>l k e V)$. This is a result of the high energy behavior of the electron collisional cross sections. ${ }^{8}$ For sufficiently large energy for both ionization and optically allowed excited state transitions $\sigma_{k}(E)$ aproaches the form

$$
\sigma_{k}(E)-A_{k} \ln (E) / E
$$


while for non-allowed transitions we have

$$
\sigma_{k}(E)-A_{k} / E
$$

asymptotically as $E$ increases.

The evaluation of $W_{k}$ still requires an estimate for $U_{k}$. Again paralleling the work of Ingkuti, we note that while Eq. 6 may be an accurate approximation. for $\mathfrak{J}_{k}(E)$ at high energies, it is obviously invalid at low energies, trivially so for $E<E_{k}$ where $J_{k}(E)$ is identically zero. Since the secondary electron spectrum for electron collisional ionization is typically peaked around $\mathrm{T}=0$ with a width of a few times $E_{i}$, we see that the substitution of Eq. 6 into Eq. 1 is valid at all positions except for the last term involving the excitation caused by the slow-down of the secondaries. Specifically if the approximation of Eq. 6 is accurate only for $E$ less than some energy $Z_{k}$ the error introduced by its substition in Eq. 1 is given by

$$
D_{k}=\sum_{i} \int_{0}^{z_{j}} p_{j}(E, T)\left[J_{k}(T)-\left(T-U_{k}\right) / W_{k}\right] d T
$$

Setting $D_{k}=0$ leads to an estimate for $U_{k}$

$$
U_{k}=\sum_{i} \int_{0}^{Z_{j}} \mathrm{p}_{i}(E, T)\left[T-W_{k} \mathrm{~J}_{k}(T)\right] d T / \sum_{i} \int_{0}^{Z_{k}} \mathrm{p}_{i}(E, T) d T
$$

At sufficiently high primary electron energy, the sriape of the 
secondary electron energy distribution function becomes independent of $E,{ }^{9}$ and $U_{k}$ (as well as $W_{k}$ ) is then well represented by a constant.

While the argument above demonstrates that $J_{k}(E)$ must asymptotically approach the functional form of Eq. 6, equations 8 and 11 are in general too imprecise to be used for the evaluation of $w_{k}$ and $U_{k}$. However, it does allow for a simpification of the solution to the Fowler equation in that rather than iterating Eq. 1 all the way to . - energy of interest, we need only proceed to suficiently large energy to obtain accurate estimates of $W_{k}$ and $U_{k}$.

The solution of the Fowler equation strictiy applies to the excitation produced in the case where the primary electron, as well as all secondaries it produces, stop in the gas. However, because the secondary electron energies are typically much smaller than that of the primary, there often exist cases where while the primary electron proceeds through the gas, the secondaries that it creates stop in it. When this is the case, we have that the excitation yields generated in the gas by a primary whose energy is degraded from $E_{j}$ to $E_{f}$ is given by

$$
J_{k}\left(E_{i}, E_{f}\right)=J_{k}\left(E_{j}\right)-J_{k}\left(E_{f}\right)
$$

Furthermore, if $E_{f}$ is large enough, we may use Eq. 6, in which case $J_{k}\left(E_{i}, E_{f}\right)=\left(E_{i}-E_{f}\right) / W_{k}$. 
B. Electron Collisional Excitation and Ionization Cross Sections

The first step in proceeding towards a solution of the Fowler equation for the excitation yields is the specification of the excited and ionic states which are important in ine stopping of electrons in the gas mixture of interest. In addition, the electron collisional cross sections for excitation of these states are required in order to determine the excitation probabilities $p_{k}(E)$.

Collections of inelastic electron collisional cross section data have been compiled and utilized by numerous workers in the study of electron slow-down in some of the lighter rare gases. 10-16 Such data sets range in complexity and accuracy, and are generally a combination of experimental measurement, empirical approximation, and theoretical extrapolation. In the selection of the cross sections for this work, we attempted to survey all of the relevant literature to come up with the most consistent and accurate approximations.

The krypton cross sections for the effective levels listed in Table I were taken from the coliection gathered by Hyde and Roberts. 10 The ionization criss section was taken from Rapp and Golden ${ }^{17}$ while the secondary electron energy distribution is that of Green and Sawada. ${ }^{9}$ For the allowed transitions the empirical cross 
sections of Ganas and Ereen were used. ${ }^{18}$ The cross section for the forbidden $4 p-5 p$ transition was assumed to be of the form

$$
\sigma_{k}(E)=A_{k}\left(1-E_{k} / E\right) / E
$$

Hyde and Roberts determined the magnitude of $A_{k}$ by requiring that the solution of the Fowler equation using their cross section data for a pure krypton gas yield the experimentally determined value for the mean energy deposited per ion pair.

For argon we chose to use the empirical cross sections of Peterson and Allen, 11 who considered ionization from both the argon $M-$ and L-shells as well as the optically allowed and forbidden transitions of the $3 p$ electron. Their ionization cross sections, secondary electron energy distributions, and composite forbidden transition cross section (identified as $3 p \rightarrow 4 p$ ) were used as given. For the allowed excited state transitions we have followed Hyde and Roberts in consolidating the levels of Peterson and Allen into the effective levels listed in Table II. ${ }^{10}$ In addition, we fitted the high energy end of the optically allowed cross sections to the proper functional form of Eq. 9.

For neon, the excited and ionic states considered are presented in Table IIl. The ionization cross sections, secondary electron energy distribution functions, and dipole allowed excitation cross sections were taken from the collection gathered by Soong. ${ }^{13}$ As for 
krypton, we included the forbidden $2 p \rightarrow 3 p$ transition, for which we used the cross section assumed by Hyde and Roberts, which has the energy dependence of Eq. 14 10 $^{10}$

Though several collections of inelastic cross section data for helium exist, ${ }^{14-16}$ we singled out no one source in making our selection of the relevant helium excited states and the cross sections for their production. The levels used in our treatment of the electron slowdown in helium are presented in Table IV along with the sources of the inelastic cross section data for transitions to these states. Figures 1-5 show the nelium inelastic cross sections used in this work. At high energies the cross section for the forbidden singlet - triplet transition was assumed to be of the form

$$
\sigma_{k}=A_{k} / E^{n} k
$$

(Ref. 28) where $A_{k}$ and $n_{k}$ were determined by fitting to the experimentally determined cross sections at the highest energies.

C. The Numerical Solution of the Fowler Equation

The numerical solution of the Fowler equation begins with the choice of a mesh of energy points $E_{n}$, followed by the evaluation of $p_{k}(E)$ and $p_{i}(E, T)$ on this mesh, and concludes with the estimation of the various $J_{k}(E)$ at each of the mesh points. Below we shall 
discuss the method of solution, and give the results for a few gas mixtures of interest.

One complication which becomes immediately apparent in finding the solution to this equation is a result of the fact that it is solved in a iterative manner, proceeding to ever higher values of the electron energy. Any errors present in the solution for $J_{k}(E)$ at small energies propagate through the iteration procedure. An accurate solution at large values of the electron energy requires that particular attention be paid to the solution close to tinreshold. This in turn requires the energy mesh to be sufficiently "fine" at the lowest relevant energies.

Computationally, however, there are two reasons why we would like to make the mesh as coarse as possible. First, a coarse mesh requires fewer evaluations of $J_{k}(E)$ to reach a given value of the electron energy. More important is the fact that in order to calculate $J_{k}(E)$ the values of all the " $p$ 's" are required at all mesh points $E_{n}$ such that $E_{n}<E$, along with all of the parameters required to specify the secondary electron energy distribution functions at the primary energies $E_{n}$. A mesh which is too fine places unacceptable storage requirements on the storage capacity of the computer chosen to carry out the solution, as well as making such a solution prohibitively expensive in terms of computer time.

We see in this way that the solution of thc Fowler equation 
requires a compromise between accuracy and expediency. The prescription which was chosen for the solution of this problem is as follows. The energy mesh was started at an energy $E_{j}$, which was less than the excitation energy of any of the states of interest. The mesh was the extended by the relation $E_{n+1}=(1+\infty) E_{n}$, which continued until the mesh size $E_{n+l}-E_{n}$ was greater than $\epsilon /(1+\alpha)$. From this paint on, the mesh was continued by setting $E_{n+1}=E_{n}+c$. In practice, the choice $E_{1}=8.00 \mathrm{eV}, \alpha=.015, \epsilon=4.00 \mathrm{eV}$ was found to be sufficient for an accurate solution of $J_{k}(E)$; further decreasing any of these parameters had a negligible effect on the solution.

Once the mesh was chosen, and the various $p_{k}(E)$ and secondary electron energy distribution parameters were calculated on this mesh, the solution required two final components: a method for the evaluation of $J_{k}(E)$ at an arbitrary energy $E$, and a technique for the integration of $J_{k}(E)$ over the primary and secondary electron energy distributions resulting from ionization. For the former, a quadratic interpolation scheme was found sufficient; for the latter a four-point integration scheme was chosen

The actual solution of the fowler equation was calculated using two computer programs. The first, CROSS, generated the energy mesh and evaluated the cross sections $\sigma_{k}$ and secondary electron distribution function parameters for all states $k$ at all the grid points. The program STOPPER then used this data to calculate $J_{k}(E)$, given the relative densities of the various gas components, and fitted 
the solutions at high energy to the form of Eq. 6. A listing of both of these programs is presented in the microfiche at the end of this thesis.

(At this point, the reader may have noticed that the above two programs allow the inclusion of a xenon component in the gas mixture, and includes cross sections for the excitation of various xenon states. The state selection and cross sections for xenon were taken from the work of Hyde and Roberts, ${ }^{10}$ and were included as a matter of completeness. In $\mathrm{KrF}^{\star}$ laser media, xenon is an unwanted impurity however, and no calculations involving xenon as a gas component will be presented in this thesis.)

A typical example of the behavior of the energy dependence of the numerical solution of the Fowler equation is showr in Fig. 6. At first, as the electron energy increases above the tireshold for the state of interest, $\theta_{k}(E)$ (defined as $J_{k}(E) / E$ ) decreases as additional channels for electron energy loss come into play. When the electron energy equals $E_{k}+E_{i}$ where $E_{j}$ is the ionization energy of any of the gas atoms, $\theta_{k}(E)$ rapidly increases as excitation of the state $k$ by post ionization electrons becomes possible. Finaliy, as the energy continues to increase, the fluctuations in $\theta_{k}(E)$ damp out as it approaches its high energy form $\left(1-U_{k} / E\right) / W_{k}$.

The high energy solution of the Fowler equation is contained in the values of $U_{k}$ and $W_{k}$. These are presented in Table $V$ for the 
pure rare gases, and in Tables VI and VII for two gas mixtures representative of krypton fluoride laser mixes. For the pure rare gases the calculated values of $W_{i}$ are of good agreement witi the experimentally determined values of the mean energy loss per ion pair created by a high energy electron ( $46 \mathrm{eV}$ for $\mathrm{He}, 37 \mathrm{eV}$ for $\mathrm{Ne}, 26 \mathrm{eV}$ for $\mathrm{Ar}$ and $24 \mathrm{eV}$ for $\mathrm{Kr}) .^{29}$ For neon and krypton this is not surprising as the magnitudes of the forbidden $\rho \rightarrow p$ cross sections were chosen by requirirg this to be the case. Although the calculated value $f \mathrm{ci}^{\circ}$ argon initially appears too large, when the Auger decay of the $\operatorname{Ar} L$-shell ion is taken into account along with the subsequent excitation of the gas by the Auger electron, it decreases to $27.1 \mathrm{eV}$, more in line with the experimental value.

\section{Discussion}

The solutions of the Fowler equation contained in Tables $y$ through VII illustrates the fundamental difference between determining the effect of the passage through a gas of a high energy electron on the electron, and determining the effect of the high energy electron on the gas. At energies above $1 \mathrm{keV}$ the primary energy loss process for the electron is ionization. Not only does the ionization cross section greatly exceed that for excitation (by a factor of 5 - 10 in the pure rare gases), but the average energy loss per ionizing collision is several times that lost in creating an excited gas atom. The values of $w_{k}$ in Table $v$ indicate however, that the number of excited states the high energy electron produces in the gas is 
typically one half the yield of ions.

This difference can be understood when one realizes that the energy lost by the primary electron in creating an ion is divided roughly equally between the ion and the secondary electron created. Because the secondary electron energy distribution is peaked at low energies, a significant fraction of these secondary electrons have insufficient energy to cause additional ionization, but are energetic enough to create a gas excited state. It is these secondary electrons which demand the complicated "bookkeeping" of the Fowler equation for the correct evaluation of the character of the gas excitation.

With this point in mind, the qualitative behavior of the variation of the $W_{k}$ with gas mixture shown in Tables $V I$ and VII can be explained. Comparing Tables $V$ and VI, we see that while the addition of a small quantity of krypton $(\approx 6.7 \%)$ to aryon has only a slight effect on the distribution of the excitation over the argon excited and ionic states, the number of krypton excited states produced now exceeds the number of krypton ions. This is a consequence of the fact that while the lowest krypton excited levels are still the lowest levels in the gas, the krypton ionization cross section must now compete with the excitation cross sections of aryon for its share of the secondary electron sper trum. This results in a reduction in the number of krypton ions formed relative to the number of krypton excited states. 
Finally we note that in mixtures of the rare gases, the higher-2 component of the gas mix receives a larger proportion of the total excitation than is indicated by its concentration. This is illustrated in Table VII, which indicates that aithough the ratio of the krypton and helium atomic densities is less than $1 \%$, greater than 25\% of the gas excitation (defined as the sum of the ionic and excited state densities) lies in krypton excitation. This is a result of two facts. First, both the cross sections for excitation and ionization by electrons increase with increasing $z$. More importantly, however, the thresholds for excitation and ionization are lower for the higher-z states, which are therefore accessible to a greater fraction of the secondary electron spectrum. 


\section{Table I}

Krypton Energy Levels Used in Electron Slowdown

$\begin{array}{lr}5 s & 9.98 \mathrm{eV} \\ 5 p & 11.47 \mathrm{eV} \\ 4 d, 6 s & 12.2 \mathrm{ev} \\ \text { Higher Neutral } & 12.85 \mathrm{ev} \\ \text { Ion } & 14.00 \mathrm{ev}\end{array}$

TABLE II

Argon Energy Levels Used in Electron Slowdowm

$\begin{array}{ll}4 \mathrm{~s} & 11.76 \mathrm{eV} \\ 4 \mathrm{p} & 13.0 \mathrm{eV} \\ 3 \mathrm{~d}, 5 \mathrm{~s} & 14.1 \mathrm{eV} \\ 4 \mathrm{~d}, 6 \mathrm{~s} & 14.96 \mathrm{eV} \\ \text { Higher Neutra) } & 15.4 \mathrm{eV} \\ \text { Ion (M-shel1) } & 15.76 \mathrm{eV} \\ \text { Ion (L-she11) } & 250 . \mathrm{ev}\end{array}$


Table III

Neon Energy Levels Used in Electron Slowdown

$\begin{array}{lr}3 s & 16.70 \mathrm{eV} \\ 3 p & 18.64 \mathrm{eV} \\ 3 \mathrm{~d}, 4 \mathrm{~s}, 5 \mathrm{~s} & 20.10 \mathrm{eV} \\ \text { Higher Neutral } & 21.21 \mathrm{eV} \\ \text { Ion (L-shel1) } & 21.60 \mathrm{eV} \\ \text { Ion (K-she11) } & 870 . \mathrm{eV}\end{array}$

Table IV

Helium Energy Levels Used in Electron Slowdown

Source of inelastic cross section data

$\begin{array}{lll}2^{3} \mathrm{~S} & 19.82 \mathrm{eV} & (\text { Ref } 19,20,21,22) \\ 2^{1} \mathrm{~S} & 20.615 \mathrm{eV} & (\text { Ref } 19,21,23,24,25) \\ 2^{3} \mathrm{P} & 20.96 \mathrm{eV} & (\operatorname{Ref} 19,21,22,26) \\ 2^{1} \mathrm{P} & 21.22 \mathrm{eV} & (\operatorname{Ref}, 24,26) \\ \text { Ion } & 24.59 \mathrm{eV} & (\text { Ref } 9,17,27)\end{array}$


Table $V$

Asymptotic Solution of the Fowler Equation for Electron Energy Deposition in the Rare Gases

Hel ium

$\begin{array}{cc}U_{k}(\mathrm{eV}) & W_{k}(\mathrm{eV}) \\ -32.4 & 691 . \\ -63.3 & 747 . \\ -47.8 & 1,500 . \\ -22.3 & 119 . \\ 16.1 & 42.6\end{array}$

Neon

$\begin{array}{cc}U_{k}(e V) & W_{k}(e V) \\ -20.2 & 174 . \\ -45.4 & 194 . \\ -18.7 & 878 . \\ -22.4 & 1,730 . \\ 1.10 & 36.8 \\ -- & 5,170 .\end{array}$

He $2^{3} s$

He $2^{1} \mathrm{~s}$

He $2^{3} p$

He $2^{\mathrm{p}} \mathrm{P}$

He Ion 
Table V (continued)

Asymptotic Solution of the Fowler Equation for Electron Energy Deposition in the Rare Gases

Argon

$\begin{array}{cc}U_{k}(\mathrm{eV}) & W_{k}(\mathrm{eV}) \\ -53.6 & 165 . \\ -78.5 & 182 . \\ -46.2 & 372 . \\ -42.2 & 1,240 . \\ -40.1 & 330 . \\ -29.3 & 29.4 \\ 532 . & 3,180 .\end{array}$

Krypton

$U_{k}(e v) \quad w_{k}(e V)$

$\mathrm{Kr} 5 \mathrm{~s}$

$-8.97$

111.

$\mathrm{Kr} 5 \mathrm{p}$

$-63.2$

208.

$\mathrm{kr} 4 \mathrm{~d}, 6 \mathrm{~s}$

$-33.9$

217.

$\mathrm{Kr}$ Higher Neutral

$-56.4$

206.

$\mathrm{Kr}$ Ion

23.0 
Table VI

\begin{abstract}
Asymptotic Solution of the Fowler Equation for Electron Energy Deposition in a $15 / 1 \mathrm{Ar} / \mathrm{Kr}$ Gas Mixture
\end{abstract}

Argon

$\begin{array}{cc}U_{k}(\mathrm{eV}) & W_{k}(\mathrm{eV}) \\ -52.5 & 246 . \\ -80.1 & 227 . \\ -44.2 & 405 . \\ -40.2 & 1,340 . \\ -38.2 & 357 . \\ -27.1 & 37.7 \\ 534 . & 3,410 .\end{array}$

Krypton

$$
\mathrm{U}_{\mathrm{k}}(\mathrm{eV}) \quad \mathrm{w}_{\mathrm{k}}(\mathrm{eV})
$$

$\mathrm{Kr} 5 \mathrm{~s}$

$-46.4$

247.

$\mathrm{Kr} 5 \mathrm{p}$

$-60.2$

851.

$\mathrm{kr} 4 \mathrm{~d}, 6 \mathrm{~s}$

$-54.4$

1,500 .

Kr Higher Neutral

$-68.2$

1,820 .

$\mathrm{Kr}$ Ion

$-10.3$

330 . 
Table VII

\section{Asymptotic Solution of the Fowler Equation for Electron Energy Deposition in a $116 / 1 \mathrm{He} / \mathrm{Kr}$ Gas Mixture}

Helium

$\begin{array}{lcr}\text { He } 2^{3} \mathrm{~S} & -37.1 & 1,800 \\ \text { He } 2^{1} \mathrm{~S} & -67.7 & 866 \\ \text { He } 2^{3} \mathrm{P} & -51.2 & 1,760 . \\ \text { He } 2^{3} \mathrm{P} & -22.0 & 130 . \\ \text { He Ion } & 17.3 & 45.2\end{array}$

Krypton

$$
U_{k}(e v) \quad w_{k}(e v)
$$$$
-37.1 \quad 1,800 \text {. }
$$$$
-67.7
$$$$
-51.2 \quad 1,760 \text {. }
$$

45.2

$\begin{array}{lcc} & U_{k}(\mathrm{eV}) & \omega_{k}(\mathrm{eV}) \\ \mathrm{Kr} 5 \mathrm{~s} & -17.7 & 272 . \\ \mathrm{Kr} 5 \mathrm{p} & -23.8 & 529 . \\ \mathrm{Kr} 4 \mathrm{~d}, 6 \mathrm{~s} & -22.2 & 833 . \\ \mathrm{Kr} \text { Higher Neutra) } & -26.7 & 833 . \\ \mathrm{Kr} \text { Ion } & -4.19 & 272 .\end{array}$




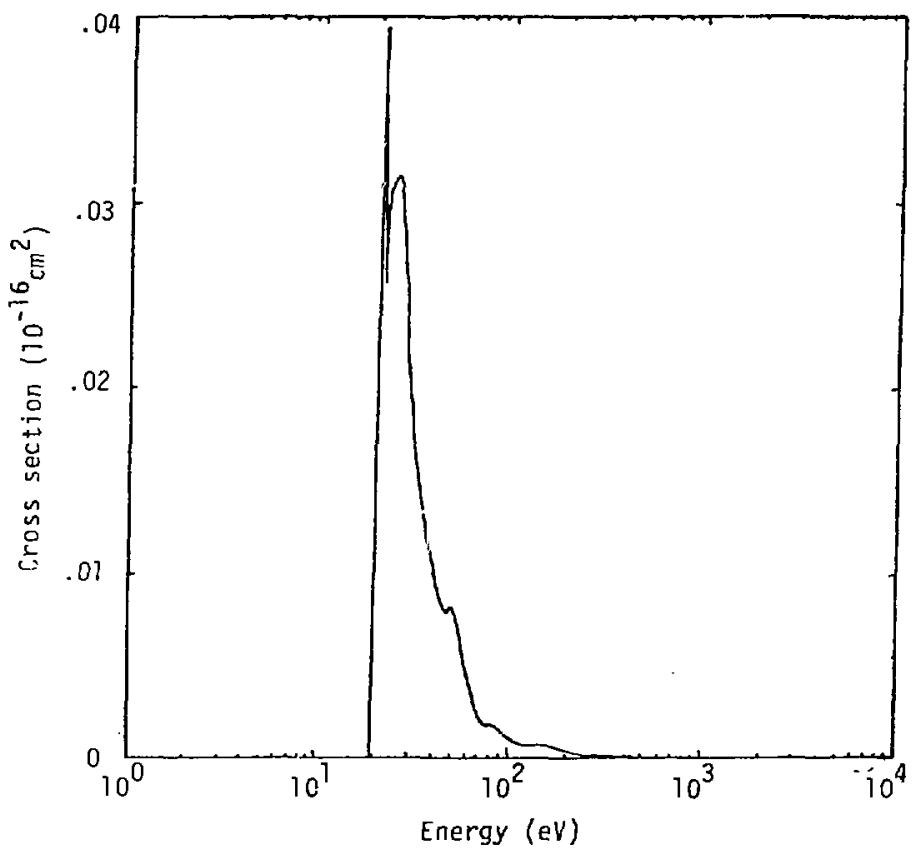

Fig. 1. Cross section vs electron energy for excitation of helium to the He $2^{3} \mathrm{~S}$ state assumed for the solution of the fowler equation 


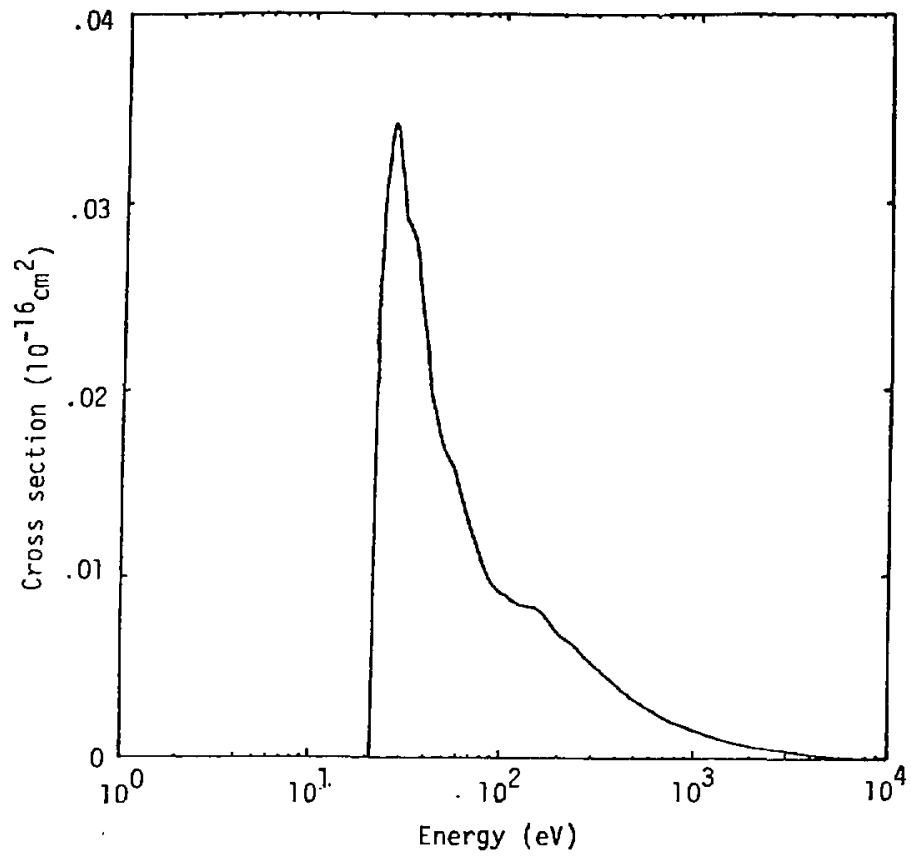

Fig. 2. Cross section vs electron energy for excitation of hel ium to the He $2^{1} \mathrm{~S}$ state assumed for the solution of the fowler equation 


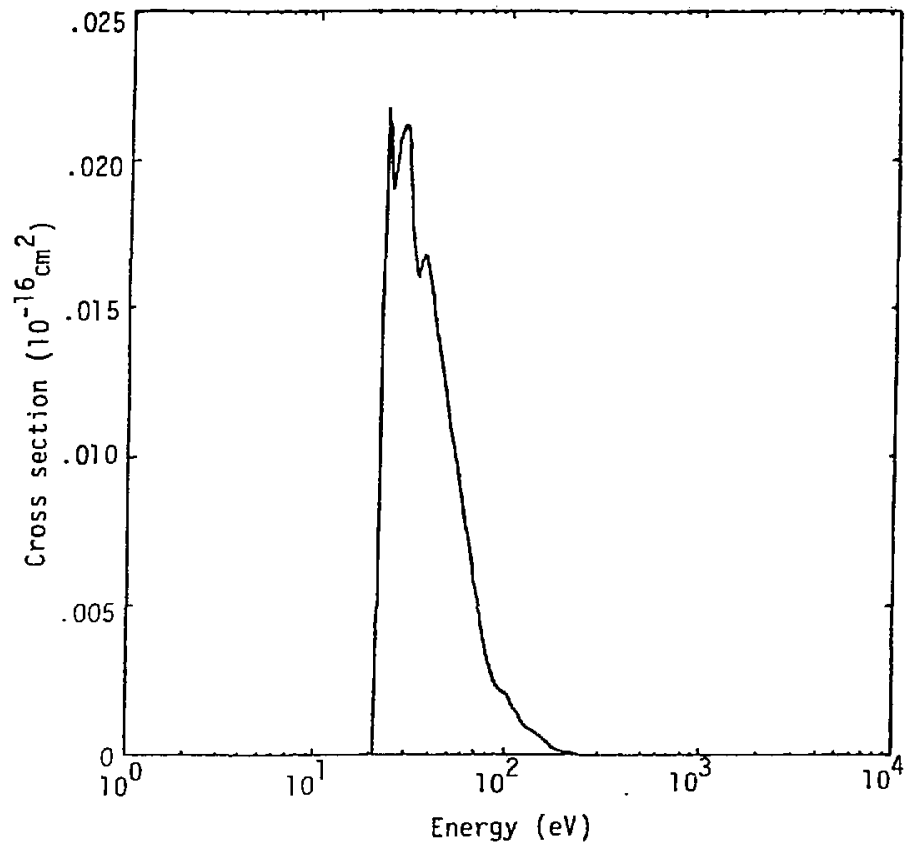

Fig. 3. Cross section vs electron energy for excitation of helium to the He $2^{3} \mathrm{P}$ state assumed for the solution of the fowler equation 


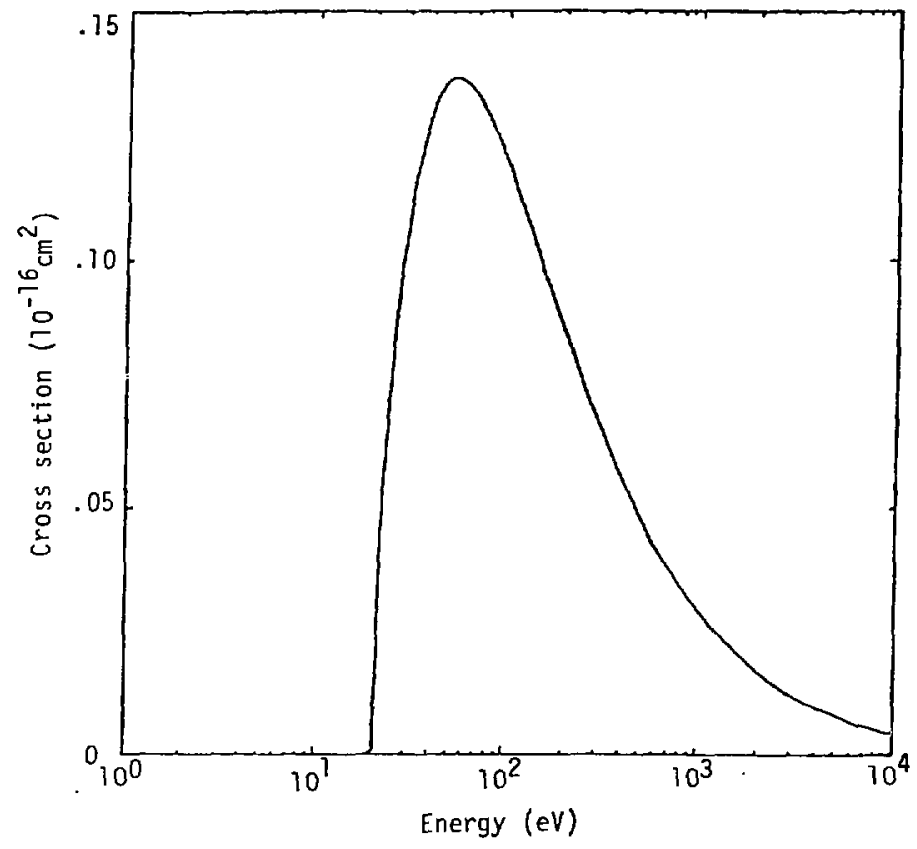

Fig. 4. Cross section vs electron energy for excitation of hel ium to the He $2^{7} P$ state assumed for the solution of the fowler equation 


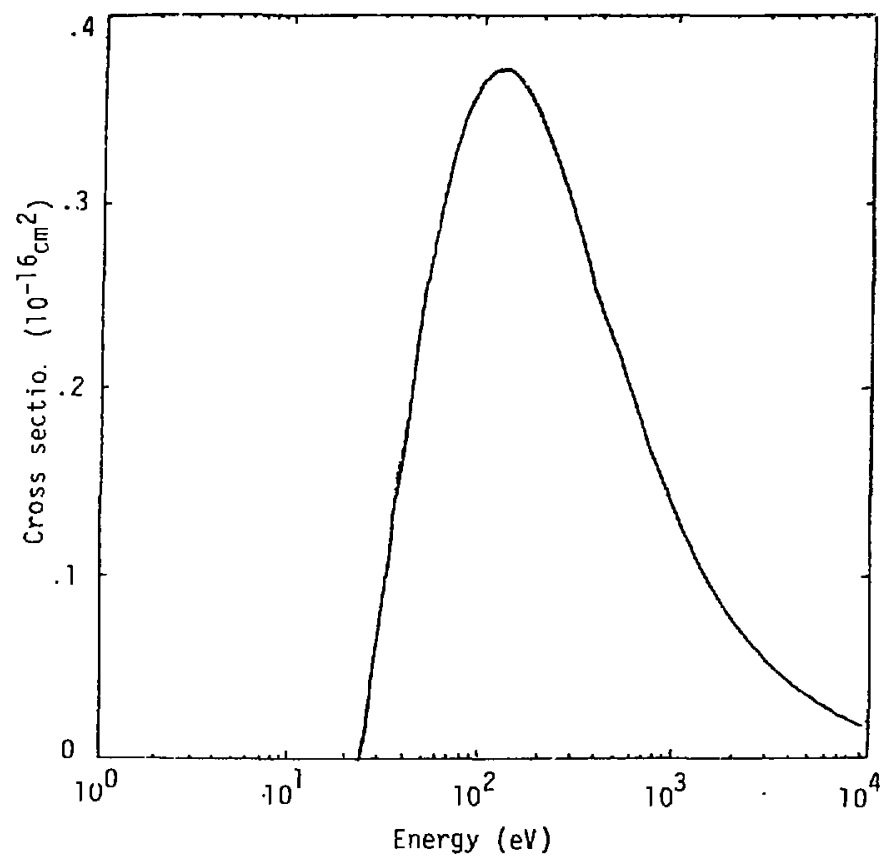

Fig. 5. Cross section vs electron energy for excitation of helium to the He ionic state assumed for the solution of the Fowler equation 


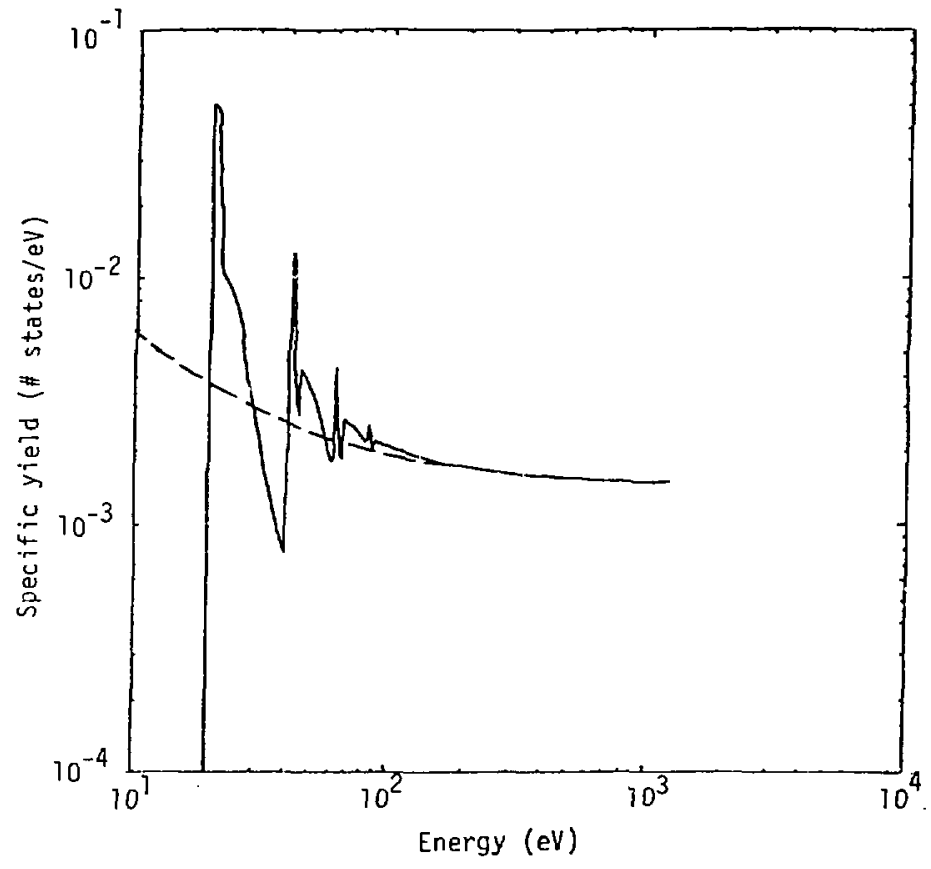

Fig. 6. Results of a Fowler equation calculation of the specific yield of He $2^{3}$ s states produced vs. electron energy by the slowing dowri of an electron in a pure helium gas. The solid line $i s$ the numerical solution; the dashed line is the fit to this solution at high energy described in the text. 
References - Chrater II

1. R. H. Fowler, Proc. Camb. Philos. Soc. 21 ,531 (1923)

2. M. J. Berger, Methods in Computational Physics (Academic, New York, 1963), Vol. I

3. U. Fano, L. V. Spencer, and M. J. Berger, in Encyclopedia of Physics, editted by S. Flügge (Springer, Berlin, 1959) Vol 38/2, p660

4. N. Bohr, Philos. Mag. 25, 10 (1917); 30, 518 (1915)

5. R. H. Garvey and A. E. S. Green,, Phys, Rev Al4, 946 (1976)

6. M. Inokuti, D. A. Douthat, and A. R. P. Rau, Proc. 5th Symposium on Microdosimetry, (1975), p977

7. M. Inokuti, Rad. Res. 64,6 (1975)

8. H. S. W. Massey and E. H. S. Burhop, Electronic and Ionic Impact Phenomena Second ed. (Cxford, 1969) Vol 1, p437

9. A. E. S. Green and T. Sawada, Jrni. Atmos. Terres. Phys. 34, 1719 (1972)

10. R. Hyde and M Roberts, UCID-1778, Lawrence Livermore Laboratory (1978)

11. L. R. Peterson and J. E. Allen, Jr., Jrn1. Chem. Phys. 56, 6068 (1972)

12. E. Eggerter, Jrn1. Chem. Phys. 62, 833 (1975)

13. S. C. Soong, Rad. Res. 67, 187 (1976)

14. Dayshankar, Physica 85C, 219 (1977)

15. W. F. Miller, Ph.D. Thesis, Purdue University (1956)

16. D. A. Douthat, Rad. Res. 61, 1 (1975) 
17. D. Rapp and P. Englander-Golden, Jrn1. Chem. Phys. 43, 1464 (1965)

18. P. S. Ganas and A. E. S. Green, Phys. Rev A4, 182 (1971)

19. R. I. Hal1, J. Reinhardt, G. Joyez, and J. Mazeau, Jrn1. Phys. B5, 66 (1972)

20. G. B. Crooks, R. D. Dubois, D. E. Golden, and M. E. Rudd, Phys. Rev. Lett. 29, 327 (1972)

21. S. Trajmar, Phys. Rev. A8, 19] (1973)

22. A. Yagashita, private communication in W. C. Fon, K. A. Berrington, P. C. Burke, and A. E. Kingston, Jrn1. Phys. B12, 1861 (1979)

23. L. Vriens, J. A. Simpson, and S. R. Mielczarek, Phys. Rev. 165, 7 (1968)

24. K. L. Bell, D. J. Kennedy, and A. E. Kingston, Jrn1. Phys. B2, 26 (1969)

25. J. K. Rice, D. G. Truhar, D. C. Cartwright, and S. Trajmar, Phys. Rev. A5, 762 (1972)

26. J. D. Jobe and R. M. St. John, Phys. Rey. 164, 117 (1967)

27. B. L. Schram, F. J. Deheer, M. J. Van der Wiel, and J. Kistemaker, Physica 31, 94 (1965)

28. H. S. W. Massey and E. H. S. Burhop, Electronic and Ionic Impact Phenomena, Second Ed., (Oxford, 1969) Vol 1, pa53

29. A. Dalgarno, Atomic and Molecular Processes, D. R. Bates ed.. (Academic Press, New York, 1962),p637 


\section{Chapter III}

The Excitation of Krypton Fluoride

Laser Media by Kilovolt X-rays

\section{A. Introduction}

Krypton fluoride lasers constructed to date have depended on the excitation of the laser media by electrons, either in a discharge or via high energy electron beam pumping. With the avvent of the inertial confinement fusion (ICF) program however, interest has grown in the possibility of pumping such lasers directly with the output of a fusion microexplosion. Bypassing the conversion of the fusion output to electrical energy would reduce both the recirculating fraction of the plant energy required to power the laser and the capital cost of plant construction.

Most of the direct pumping proposals so far have focussed on the excitation of the laser by neutrons, relying on the fact that the bulk of the energy from a first generation ICF plant driven by the D-T fusion reaction would reside in $14 \mathrm{MeV}$ neutrons. ${ }^{1,2}$ The low value of the neutron reaction cross sections for most materials however necessitates the doping of the laser medium with a suitable neutron 
absorber (e. g. boron or uranium) in high concentrations, which invariably degrade the laser performance.

Work done on possible second generation ICF power plants (specifically those which would burn "isotopically self sufficient" fuel pellets, requring no tritium breeding in the reactor vallis ${ }^{3}$ ) has indicated that a significant fraction of the fusion energy in such plants could reside in multi-kilovolt $X$-rays. Such schemes require the compression of the fusion fuel to a pr product (defined as the product of the fuel density and the characteristic pellet length at the time the fusion reaction takes place) of $\geq 5 \mathrm{gm} / \mathrm{cm}^{2}$. At these high values of pr a significant fraciion of the fusion neutron energy would redeposit in the fusion plasma. More importantiy, however, such high values of $\rho r$ would require the bremmstrablung pho ins generated in the pellet to undergo mutiple inverse Compton scatterings with the thermal electrons, by which they would be rapidly amplified in energy to a value jusi below that of the electrans (typically somewhere in the 20 - $60 \mathrm{keV}$ energy range). Adjustment of the bremmstrahlung yield by doping the fusion fuel with high-Z materials could lead to as much as $30 \%$ of the fusion energy leaving the plasma as multi-kilovolt X-rays.

In Figure 1 we present the time-integrated $x$-ray yield calculated in the computer simulation of the burn of such a high pr pellet." The spectrum is dominated by two peaks, ai $x$-ray energies of about 1 and $40 \mathrm{keV}$. The low energy peak is due to the thermal bremmstrahlung 
emitted during the expansion and cooling of the fuel plasma; the high energy peak comes from the Compton-hardened bremmstrahlung spectrum described in the preceeding paragraph.

In what follows we will first describe the physical processes which we believe to be important in the excitation of rare gas mixtures by kilovolt $x$-rays. This will be followed by a description of the computational techniques used to calculate the effect of an $x$-ray flux on specific gas mixtures. It will be demorstrated that in many ways the pumping of krypton fluoride laser media by kilovolt $x$-rays is similar to their pumping by kilovolt electrons.

\section{B. Physical Processes}

The dominant interaction of $x$-rays in the energy range of interest $(1-100 \mathrm{keV}$ ) in the higher- $z$ rare gases (i. e., other than helium) is by photoionization. The qualitative behavior of the interaction is displayed in Fig. 2, showing the variation of the total photon interaction cross section of krypton as a function of the $x$-ray energy. ${ }^{5}$ As the photon energy increases the ionization of more tightly bound eiectron shells becomes pussible, leading to discontinuous jumps in the total cross section (K-edge, L-edges, etc.). Between the edges the cross section decreases inversely as the third power of the photon energy, characteristic of the photoionization process. 6 
Due to the rapid decrease in the photoionization cross section with decreasing atomic number, additional processes must be taken into account when considering the interaction of the $x$-ray spectrum with helium. As Fig. 3 indicates, photoionization, incoherent scattering, and coherent scattering (scattering the photon with no energy loss) all contribute tu the total interaction cross section ${ }^{5}$

Returning to the heavier rare gases $(2>2)$, after photoionization the energy initially present in the $x$-ray is shared between the ion and the ionization electron. The fraction of the energy residing in the ion is not necessarily negligible, especially for the inner shells of the heavier gases. A $30 \mathrm{keV}$ X-ray will interact with krypton primarily through the creation of a $K$ - shell ion with an energy of $14.3 \mathrm{keV}$ or about $48 \%$ of the energy of the incident $\mathrm{x}$-ray.

The inner shell hole resulting from a photoionization event can decay via either of two modes, fluorescence or the ejection of an Auger electron. For the lower-z ions, and for the outer shells of the higher- $Z$ atoms, Auger decay dominates; for the high- $Z$ inner shell holes the fluorescence probabilties are appreciable (e. g., the probability of the decay of the krypton $K$-shell ion via fluorescence is $\approx 65 \%)$. $^{7}$ The fluorescence photons produced in this fashion propagate through the gas forming an additional contribution to the total $X$-ray flux seen by the medium. 
The decay of the inner shell ion, beginning with the initial fluorescence or Auger event, rapidly continues via either additional Auger emission or by shakeoff of outer shell electrons. The cascade continues downward in energy unt $i$ a stable charge state configuration is reached. The final configurations resulting from the excitation of a given initial hole have been measured and calculated.

At this point the energy originally present in the incident $X$-ray resides in multiply charged gas ions, and in the electrons produced as a result of the initial ionization and decay of the inner shell holes. The multiply charged ions themselves decay via charge stripping reactions with the neutral gas atoms, converting in the process a fraction of their energy into gas thermal enargy. We estimate that the cross section for a reaction of the form

$$
x^{n+}+y \rightarrow x^{\left(n_{-}-1\right)+}+y^{+}
$$

(where $X$ and $Y$ represent any of the rare gases, e. g. Ne, Ar, etc.) has a value proportional to the square of the interatomic radius at which the potential curves for the initial and final states cross, i. e.:

$$
\sigma \propto R^{2} \propto\left\{\frac{n-1}{E_{n}}\right\}^{2}
$$

where $E_{n}$ is the ionization potential of the $x^{(n-1)+}$ atomic state. $^{13}$ With this assumption the distribution of singly charged 
States resulting from the decay of the multiply-charged ions may be determined. This crude model for the charge exchange process is mitigated by the fact that in most of the cases of interest $\leqslant 1 \%$ of the initial $x$-ray energy finds its way into multiply charged ions, as will be demonstrated in Section $C$.

The final step in the excitation of the gas by the $x$-ray flux is the degradation of the electrons produced in the various processes described above. In this we are aided by the fact that most of the electron energy resides in high energy electrons, and that the average electron energy is typically $>1 \mathrm{keV}$. In this case the excitation of the gas by the electroris may be calculated using the high energy solutions of the fowler equation for the gas mix of interest as described in Chapter II.

\section{Numerical Solution of the $x$-ray Deposition Problem}

The determination of the gas excitation produced by an incident $x \cdot$ ray flux requires first and foremost a method for calculating the spatial transport of the $X$-ray spectrum. This was accomplished using tie TARTNP photonics code, developed at LLNL. ${ }^{14}$ Given the definition of ine problem geometry (including the material composition) and an incident $x$-ray flux, the TARTNP program uses a Honte Carlo approach to propagate tine photons, both incident and generated by fluorescence, taking into account coherent and incoherent 
scattering and photoionization. The output of this program is a spatially dependent reaction spectrum, giving the number of photons of each energy which react in each problem zone.

The program XRAYDEP (presented in the microfiche at the end of this thesis) uses this reaction spectrum to calculate the spatially dependent gas excitation, according to the prescription of Section $B$. In addition to the $x$-ray interaction data, and the inner shell vacancy cascade data described above the construction of this program made use of literature values for the energies of the various multiply charged ions. ${ }^{15,16}$ In order to determine the excitation of the gas produced by the high energy electrons, XRAYDEP also requires the results of a STOPPER calculation (described in Chapter II) for the asymptotic solutions of the Fowler equation for the gas mix in the zone of interest. In calculating the spatially dependent gas excitation, XRAYDEP assumes that the electrons deposit all their energy locally, which is reasonable as the electron ranges at the energies of interest are much shorter than those of the $X$-rays.

The solution for the deposition in a realistic ICF geometry is well beyond the scope of this thesis. The more important results of such a calculation may be equally well demonstrated in the solution of a simpler problem. To this end we chose the geometry shown in Fig. 4. A monoenergetic $30 \mathrm{keV} X$-ray source was assumed incident on a 1 meter long, 1 meter diameter 50 Torr krypton, 750 Torr argon gas mixture. The X-ray flux of $7.8 \times 10^{15}$ per $\mathrm{cm}^{3}$ is typical of what 
might be found at the inner wall of a second generation ICF reaction chamber.

In this geometry we specified as a "typical" region a $10 \mathrm{~cm}$. long, $10 \mathrm{~cm}$. diameter cylinder at the center of the gas volume; all results quoted are for this region. The disposition of the gas excitation at various points in the calculation is presented in Tables I and II. In Table I, we note that the energy present in multiply charged ians is $\approx 1 \%$ of the total deposited, and that the average electron energy is $3.13 \mathrm{keV}$, justifying the approximations for the fate of these energies described in the preceeding section.

The excitation of the gas produced by the X-rays may be converted into a set of $W_{k}$ similar to those calculated for electron excitation by dividing the results of Table II by the total X-ray energy deposited; these values are shown in Table III. Comparing this Table to Table VI in Chapter II we see that the yields of the various states produced by $x$-ray and high energy electron excitation are quie similar.

There is, however, one caveat which should be made before taking such a similarity too seriously. The difference in the magnitude of the $x$-ray and electron interaction cross sections means that both the local magnitude and the spatial variation of the energy deposition will be different for the two modes of pumping. Pumping with $X$-rays generally takes place over larger volumes, and allows a larger energy flux without overdriving the laser medium. 
Table I

Energy Deposition in a 50 Torr Kr, 750 Torr Ar Gas Mixture by $30 \mathrm{keV}$ X-rays

Initially...

Energy deposited by $x$-rays $\quad 1.74 \times 10^{18} \mathrm{eV} / \mathrm{cm}^{3}$

Energy loss due to fluorescence $0.07 \times 10^{18} \mathrm{eV} / \mathrm{cm}^{3}$

Net energy deposition $\quad 1.67 \times 10^{18} \mathrm{eV} / \mathrm{cm}^{3}$

After the decay of the inner shell holes...

Energy in multiply charged ions $1.97 \times 10^{16} \mathrm{eV} / \mathrm{cm}^{3}$

Total energy in electrons $\quad 1.65 \times 10^{18} \mathrm{ev} / \mathrm{cm}^{3}$

Average electron energy $\quad 3.13 \mathrm{keV}$ 


\section{Table II}

\section{Excitation of a 50 Torr $\mathrm{Kr}, 750$ Torr $\mathrm{Ar}$ Mixture by $30 \mathrm{keV} X$-rays}

Excited State and Ion Densities

$\begin{array}{ll}\text { Argon } & \left(\# / \mathrm{cm}^{3}\right) \\ \text { Ar 4s } & 7.16 \times 10^{15} \\ \text { Ar 4p } & 7.81 \times 10^{16} \\ \text { Ar 3d,5s } & 4.34 \times 10^{15} \\ \text { Ar 4d,6s } & 1.31 \times 10^{15} \\ \text { Ar Higher Neutra] } & 4.92 \times 10^{15} \\ \text { Ar Ion (M-she11) } & 5.65 \times 10^{16} \\ & \\ \text { Krypton } & \left(\# / \mathrm{cm}^{3}\right) \\ \text { Kr 5s } & 7.14 \times 10^{15} \\ \text { Kr 5p } & 2.07 \times 10^{15} \\ \text { Kr 4d,6s } & 1.17 \times 10^{15} \\ \text { Kr Higher Neutral } & 9.74 \times 10^{14} \\ \text { Kr Ion } & 5.41 \times 10^{15}\end{array}$


Effective Values of $W_{k}$ for the Excitation of a 50 Torr $\mathrm{Kr}, 750$ Torr Ar Gas Mixture with $30 \mathrm{keV} X$-rays

$$
\text { Argon }
$$

Ar 45

Ar $4 p$

Ar $3 d, 5 s$

Ar $4 d, 6 s$

Ar Higher NeL $\pm r a]$

Ar Ion (M-sheil)

Krypton

$\mathrm{Kr} 5 \mathrm{~s}$

$\mathrm{Kr} 5 \mathrm{p}$

$\mathrm{Kr} 4 d, 6 s$

$\mathrm{Kr}$ Higher Neutral

$\mathrm{Kr}$ Ion
$W_{k}(\mathrm{eV})$

233.

213.

384.

1,274 .

339.

29.5

$W_{k}(\mathrm{eV})$

234.

804.

1,420 .

1,710

308. 


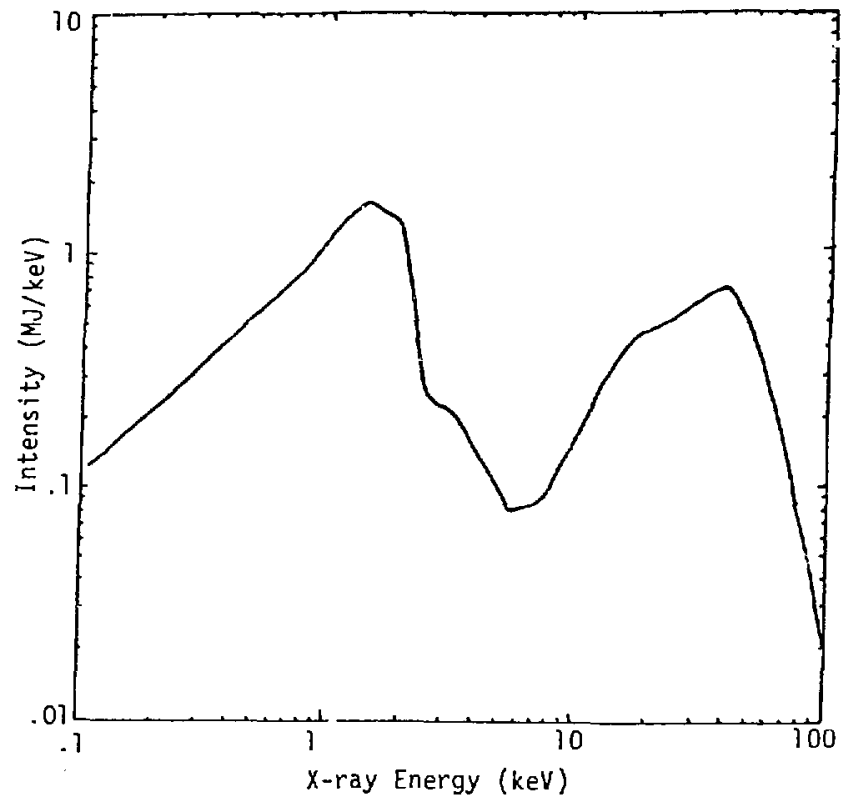

Fig. 1. X-ray energy spectrum calculated by the computer simulation of the burn of a high or ICF fuel pellet ${ }^{5}$ 


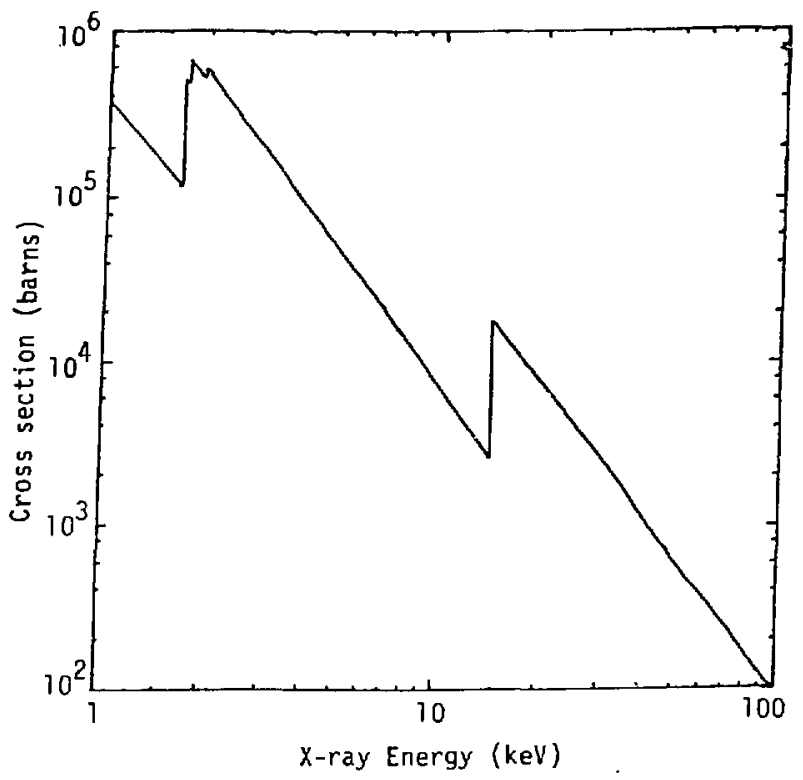

Fig. 2. Total photon interaction cross section of krypton vs. phoion energy. 4 


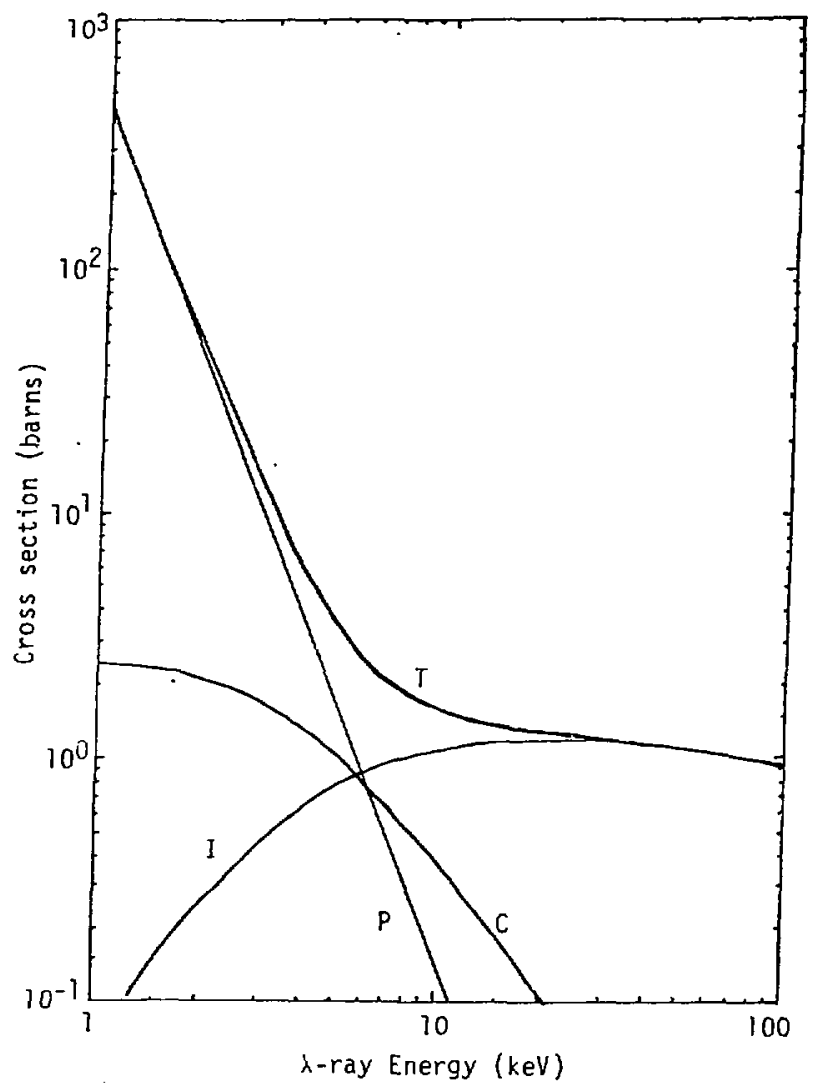

Fig. 3. Helium $X$-ray scattering cross sections vs photon energy: $T$ - total cross section, 1 - incoherent cross section, $P$ - photoionization cross section, $C$ - coherent cross section 


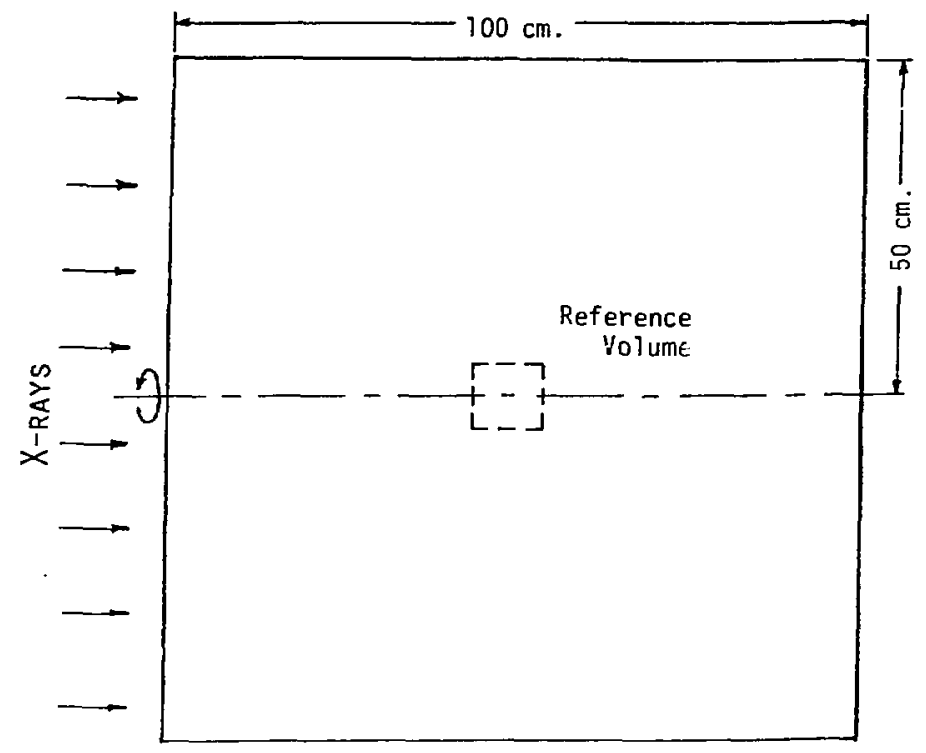

Fig. 4. X-ray gas excitation, geometry chosen for the iilustration of the solution of the energy deposition. The $x$-ray flux $\left(7.85 \times 10^{15} \mathrm{~cm}^{-3}, 30 \mathrm{kev}\right)$ is incident on a 1 meter long, 1 meter diameter cylinder containing a 50 Torr $\mathrm{kr}, 750$ Torr Ar gas mixture. The reference volume is a $10 \mathrm{~cm}$. long, $10 \mathrm{~cm}$. diameter cylinder in the middle of the gas; all results quoted in the text refer to the deposition in this volume. 
$\underline{\text { References - Chapter III }}$

1. W. E. Wells, Report of Workshop on Direct Nuclear Pumping of Lasers (1976) pl17

2. ․ Wilson, Report of Workshop on Direct Nuclear Pumping of Lasers (1976) p209

3. K. Jancaitis, J. Maniscalco, J. Hovingh, L. Wood, J. Nuckolls, J. Pettibone, and T. Weaver, UCRL-77058, Lawrence Livermore Laboratory (1975)

4. W. H. McMaster, N. Kerr Del Grande, J. H. Mollett, and J. H. Hubbel, UCRL-50174 Sec. II Rev. I, Lawrence Livemore Laboratory (1969)

5. L. Wood, Applications of Rare Gas Halides and HF Lasers Workshop, WJSA-78-6-SR3 (W. J. Schaffer Associates, Inc., 1978) pW0-21

6. J. B. Hasted, Physics of Atomic Collisions (American Elsevier, New York, 1972) p500

7. E. F. Plechaty, D. E Cullen, and R. J. Howerton, UCRL-504nn Vol. 6 Rev. I, Lawrence Livermore Laboratory (1975)

8. M. 0. Krause, M. L. Vestal, W. H. Johnston, and T. A. Carlson, Phys. Rev. 133, A385 (1964)

9. T. A. Carlson and M. 0. Krause, Phys. Rev. 137, Al655 (1965)

10. M. O. Krause and T. A. Carlson, Phys. Rev. 149, 52 (1966)

11. T. A. Carlson, W. E. Hunt, and M. 0. Krause, Phys. Rev. 151, 41 (1966) 
12. M. 0. Krause and T. A. Carlson, Phys. Rev. 158, 18 (1967)

13. J. B. Hasted and A. Y. Chong, Proc. Phys. Soc. 80, 441 (1962)

14. E. F. Plechaty and J. R. Kimlinger, UCRL-504000 Vol. 14, Lawrence Livermore Laboratory (1975)

15. C. W. Allen, Astrophysical Quantities, (Athlone Press, London, 1973) p36

16. R. Kelly and D. Harrison, Atomic Data $\underline{3}, 177$ (1971) 
Chapter IV

Krypton Fluoride Laser Kinetics

\section{A. Introduction}

The two previous chapters have shown how the energy of the laser driving mechanism (either high energy electrons or multi-kilovolt $x$-rays) is transferred to the laser medium in the form of ions, excited gas atoms, gas thermal energy, and sub-excitation (i.e. with an average energy which is below the lowest excitation energy of the ground state gas atoms) electrons. We now determine how tlis initial gas excitation is channeled into the iaser upper state and then into the laser output spectrum.

The chemical nature of the $\mathrm{KrF}^{\star}$ excimer laser system, described in Chapter I, requires that the initial gas excitation pass into the KrF* upper state through a series of two- and three-particle reactions. In Section B we identify the atomic and molecular species ano the reaction processes relevant to a description of the formation and destruction kinetics for the $\mathrm{KrF}^{*}$ excimer. The rates at which these reactions take place are estimated, and the approximations utilized in the construction of the kinetic model are discussed. 
The interaction between the radiation field and the gas components is the last step in the conversion of the energy of the driver into laser energy, and is treated in Section $C$. The bound-free nature of the radiative transitions typical of excimer lasers leads to a unique spectral line shape which is a function of the gas temperature. In addition to stimulated and spontaneous emission, absorption of the laser photons by the gas medium must be considered in the determination of the laser efficiency.

The numerical methods used to integrate the laser kinetic equations are presented in Section $D$. Not only the time dependence of the various quantities, but also the spatial variations caused by the propagation of the laser beam must be taken into account. Finally, the solution of the kinetics problem for some simple cases aie computed and compared with experiment.

\section{B. Particle Kinetics}

Excited atoms, atomic ions, dimer and trimer molecular excimers and ions, and electrons all play important roles in the formation and destruction of the $\mathrm{KrF}^{\star}$ upper state, each to varying degrees according to the composition of the laser medium and the strength of the excitation source. In Table I we present a list of the species which we have determined are relevant to the description of the particle kinetics in electron-beam (or $X-r a y$ ) excited mixtures of helium, argon, krypton, and fluorine, along with the energies of each of these 
states relative to their unexcited gas components. For argon we have assumed that the more energetic excited states (e.g. Ar 3d,5s) created in the gas by the high energy electrons (both directly and indirectly) rapidiy cascade down in energy to the Ar 4p level, in line with other. treatments of rare gas kinetics. ${ }^{1,2}$ A similar rate has been assigned to the krypton excited states above the $5 p$ level. In both cases the energy released in the cascade is presumed to corvert rapidly into gas thermal energy.

Table II is $c$ list of the gas reactions considered in the model for the krypton fluoride laser kinetics. Comparison of the various reaction rates under typical operating conditions (e.g. a 750 torr Ar, 100 torr $\mathrm{kr}, 5$ torr $F_{2}$ gas mixture with an energy deposition of $.5 \mathrm{MW} / \mathrm{cm}^{3}$ from a high energy e-beam) indicates that the excitation intially present in the excited gas atoms and ions is converted with $\geqslant 90 \%$ efficiency into the K:F* upper state. The energy conversion efficency is typically 20-30\%, resulting from the disparity in the energies of the atomic states and the KrF* excimer. This high conversion efficiency does not however allow the details of the formation kinetics to be ignored. Many of the precursors to the formation of $\mathrm{KrF}^{\star}$ (e.g. $\mathrm{Kr}_{2}^{+}, \mathrm{Ar}_{2}^{+}$, etc.) have significant photabsorption cross sections at the laser wavelength (248 nm.) as will be discussed in Section $C$. The particular path which the excitation takes to reach the $\mathrm{KrF}$ * upper state thus affects the net gain coefficent of the gas medium. Similarly, the post-KrF* formation 
kinetics must be followed as the products of the upper state destruction can also be strong absorbers (e.g. $\left.\mathrm{Kr}_{2} \mathrm{~F}^{\star}\right)$.

While many of the reaction rates listed in Table II have been determined experimentally, others have been estimated using various theoretical approximations. A full description of the kinetics model therefore requires in addition to the Tables a discussion of the assumptions which went into their construction. This information is presented in the following paragraphs.

The most important assumption implicit in Table II is that the electron energy distribution may be represented by a Maxwellian at some temperature $T_{e}$ (which is in general different from the gas temperature $T_{g}$ ). While the validity of this assumption has not been proven in general, solutions of the Boltzman equation for the energy distribution function under conditions relevant to electron beam pumping of krypton fluoride laser media have shown that a Maxwellian distribution is a reasonable approximation. ${ }^{3,4}$ The generation of "holes" in the distribution by the inelastic processes is prevented due to the presence of the super-elastic channels.

For electron collsional excitation and ionization processes where the rate constants were not known the two-temperature model allowed their calculation as a function of the electron temperature via the integration : the corresponding cross section over the Maxwellian velocity distribution. The super-elastic rate constants were 
determined using the principle of detailed balance. ${ }^{5}$ The electron-atom thermal equlibration rate (from elastic collisions) was calculated from the momentum transfer cross sections using the formulation of Shkarofsky et. al. ${ }^{6}$ For the electron-ion thermal energy exchange rate the result given by Spitzer for a partially ionized gas was used. ${ }^{7}$ In all cases the results were fit to a suitable expansion in terms of the electron temperature.

For exothermic bimolecular reactions of the form $A+B \rightarrow$ Products, the temperature dependence of the rate constant, if not known from experiment, was estimated by assuming that the corresponding cross section was roughly constant over the relevant energy range, in which case the rate scales as $T_{g}^{.5} \cdot 8$ For ternary reactions of the form $A+B+C \rightarrow A B+C$ the reaction was pictured as consisting of two callisions: first of $A$ and $B$ to form the product in an unstable state, followed by a stabilizing collision with $C .{ }^{9}$ In this case the rate again scales as $T_{g}^{.5}$, and as a function of the masses $m_{A B}$ and $m_{C}$ it is proportional to $\left(1 / m_{A B}+1 / m_{C}\right)^{\cdot 5}$. This last result was used to estimate the value of unknown ternary reaction rates in terms of known values.

For the recombination of the rare gas ions with $F^{-}$the calculation of Flannery and Yang (for $\mathrm{Ar}^{+}+\mathrm{F}^{-}$in Ar, etc.) ${ }^{10}$ was extended to other rare gas ions (e.g. $\mathrm{Ar}_{2}^{+}$) and other buffer gases (e.g. $\mathrm{Ar}^{+}+\mathrm{F}^{-}$ in $\mathrm{He}$ ). The results of this calculation reduce to the Thomson theory at low pressure $(<1$ atm.) and that of Langevin at high pressure ( $>10$ 
atm.), 17 and required both values for the polarizability of the buffer gases $^{12}$ and the reduced mobilities of the ions in the buffers. ${ }^{13-15}$ For ion-buffer pairs where the mobility was not known it was scaled from known values in the same buffer by assuming the reduced mobilty to be proportional to the ion-buffer atom reduced mass; for gas mixtures the mobility was calulated using Blanc's law. ${ }^{12}$

Two assumptions have been made concerning the behavior of the collisionally formed $\mathrm{KrF}^{\star}$ dimer. First, the shape of the radiative decay spectra measured by Murray and Powel ${ }^{16}$ indicate that the relaxation of the vibrational excitation to a thermal distribution at the gas temperature is rapid at pressures of interest for electron beam pumped lasers $(\geqslant l \mathrm{~atm})$; we assume that it takes place instaneously. Furthermore, we assume that the $\mathrm{KrF}^{*}$ excitation is, divided equally between the $B$ and $C$ states, so that only half of it is avilable to the laser transition.

Where neither the rate constant nor the cross section for a particular process was kiown (from either theory or experiment), the rates were approximated in various ways. For electron collisional ionization of the Ar $4 p$ excited state, the cross section was assumed to have the semi-empirical shape suggested by Green and Barth. 17 When the rate constant for a similar process was available, the unknown rate constant was assumed equal to it. In the case of the fluorine excited state reactions, all processes were assumed to take place at their gas kinetic values. 
C. Interaction of the Radiation Field with the Laser Medium

(i) Spontaneous and Stimulated Emission

The calculation of the strength of the KrF* laser transition requires first of all a description of the wavefunctions of the upper and lower states it connects and the magnitude of the transition moment between them. This problem is most easily treated in the Born-0ppenheimer approximation, 106 in which the molecular state is expressed as a product of an electronic and a nuclear wavefunction:

$$
|\phi\rangle=\left|\phi_{e}\right\rangle\left|\Phi_{n}\right\rangle
$$

ignoring terms on the order of the ratio of the electronic and nuclear masses, the Schrodinger equation for the molecular wavefunction becomes separable. The eigenvalues of the electronic Hamiltonian are then dependent on the inter-nuclear separation $R$, contributing to the potential for which the nuclear wavefunction must be solved.

Hay and Dunning have performed ab initio calculations for the electronic states of $\mathrm{KrF}^{\star}$, obtaining both the nuclear potential curves and the transition moments for the states relevant to the $248 \mathrm{~nm}$. krypton fluoride laser transition. 107 Figure 1 shows the potential curves for the $B\left(\frac{l}{2}\right)$ and $x\left(\frac{l}{2}\right)$ states involved. The eigenfunctions $|v\rangle$ for the upper state potential curve are bound, with a vibrational spacing of about $.042 \mathrm{eV}$. The repulsive nature of the lower state 
potential leads to a continuum of unbound states $|\epsilon\rangle$ which are normalized by requiring $\left\langle\epsilon^{\prime} \mid \epsilon\right\rangle=\delta\left(\epsilon^{\prime}-\epsilon\right)$.

The total radiative linest ape is the sum of the contributions from each of the vibrational ciqensitates to the continu.'m of lower state energy levels. For any sinyle vibrationai state the spontaneous decay rate with the emission of a photon of frequency $y$ is given by

$$
A_{v}(\nu)=\frac{64 \pi^{4}}{3 \lambda^{3}}|\langle\epsilon|t\langle R\rangle| v\rangle|^{2}
$$

where $h \nu$ is the energy difference between the initial and final state energies, $\lambda=c / \nu$, and $t(R)$, d-ifined as

$$
t(R)=\left\langle\phi_{e}^{u}\left|\frac{\vec{d} \cdot \vec{R}}{R}\right| \phi_{e}^{l}\right\rangle
$$

is the electronic dipole moment between the initial and final states. 108 The stimulated emission cross section at the frequency may be calculated fron the decay rate using the relation

$$
\sigma_{v}(\nu)=\frac{\lambda^{2}}{8 \pi} A_{v}(\nu)=\frac{8 \pi^{3}}{3 \lambda}|\langle\epsilon|t(R)| v\rangle|^{2}
$$

In thermal equilibrium at the temperature $T_{y}$ the relative population of each of the vibrational upper states is given by

$$
n(v)=\exp \left(-E_{v} / k T_{g}\right) / \sum_{v} \exp \left(-E_{v} / k T_{g}\right)
$$


where $E_{v}$ is the energy of the vibrational level $|v\rangle$. The total radiative decay rate into the continuum with the emission of a photon of frequency $\nu i$, then equal to

$$
A(\nu)=\sum_{V} \exp \left(-E_{v} / k T_{g}\right) A_{v}(\nu) / \sum_{v} \exp \left(-E_{v} / k T_{g}\right)
$$

with a similar relation holding for the total stimulated emission cross section as a function of the frequency.

The program SPECTRA, developed by the TAMP group at $L L L^{109}$ was used to calculats the factors $A_{\nu}(\nu)$ for the $\mathrm{KrF}^{\star} B \rightarrow \mathrm{X}$ laser transition, using the potential energy curves and transition moments of Hay and Dunning as input. These were combined with the proper weighting to calcuiste the spontaneous emission probabilty $A^{\prime}, \nu$ ) as well as the stimulated emisizion cross section as a function of temperature. The results of this calculation: are illustrated in Figures 2 and 3 . The tendency of both quantities to "blue shift" with increasing temperature is a consequence of the increasing contribution of the higher vibrational states via the Boltzman factor. Not only doe: the energy of the photon from a transition to a lower state of a given energy $\epsilon$ increase due to the greater energy of the vibrational state, but the higher lyin; states extend to larger values of the inter-nuclear separation, overlapping unbound states of lower energy which leads to even larger photon energies. 


\section{(ii) Absorption}

It is not simply the magnitude of the absorption cross section which must be taken into account in determining the optical loss in the gas but rather its product with the expected concentration of the absorber in the medium at the time of laser action. The species causing significant absorption of the laser radiation are presented in Table III, along with the absorption cross sections at $248 \mathrm{~nm}$. All of the absorption processes are broad band, corresponding to photodetachment, photoionization, or photodissociation.

The $\mathrm{Ar}_{2}^{+}$and $\mathrm{Kr}_{2}^{+}$absorptions have been identified as bound-free transitions, similar in nature to the $\mathrm{KrF}^{*} B \rightarrow X$ stimulated emission. The program SPECTRA was used to calculate the vibrational line shapes from the potential curves and transition moments of Michels et al, 114 which were then combined to determine the absorption cross sections as a function of wavelength and temperature. The variation of the cross sections with frequency at $300 \mathrm{~K}$ is presented in Figure 4 . The 248 nm. absorption, lying in the wings of both curves, rises with increasing temperature as the contribution to the total cross section from the higher lying vibrational states increases.

The cross sections for photon absorption of both the rare gas excimers (e.g. $\mathrm{Ar}_{2}{ }^{\star}$ ) and the rare gas halide trimers (e.g. $A r_{2} \mathrm{~F}^{\star}$ ) were estimated as equal to that of the corresponding rare gas dimer ion. The excimers are Rydberg states consisting of an outer electron around a dimer ion core; the trimers are essentially ionic complexes of the 
form $\mathrm{Ar}_{2}^{+} \mathrm{F}^{-}$. In both cases we would expect the absorption at $248 \mathrm{~nm}$. to be similar to that of the dimer ion.

\section{Numerical Methods}

Unlike the particle concentrations, whose kinetics involve simple differential equations, the growth of the flux of laser photons $\phi$ is determined by a partial differential equation in both space and time:

$$
\frac{1}{c} \frac{\partial}{\partial t} \phi=-\frac{\partial}{\partial x} \phi+\alpha \phi
$$

where $c$ is the light velocity in the laser medium, and $\boldsymbol{\alpha}$ is the net gain coefficient at the laser wavelength. Under conditions where the integral of the gain coefficient over the laser path length exceeds unity, the laser flux varies significantly with position and may induce corresponding variations in the particle concentrations. An accurate description of the kinetics in even its simplest non-trivial cases requires a prescription for transporting the laser photons according to Eq. 7 and must allow for spatial as well as temporal variations in all relevant quantities.

The most direct approach would be to integrate Eq. 7 numerically in both space and time, where for accuracy the spatial step in the integration would be limited to values $\ll \alpha^{-1}$. For large strongly pumped systems (such as the LLNL B-amplifier, which is expected to have a small signal gain of between $e^{10}$ anc ${ }^{15}$ ), with lengths much 
greater than $\boldsymbol{\alpha}^{-1}$, the number of grid points required makes such schemes computationally unfeasable. To treat such cases, an aprroximation to the spatial integral must be used.

The method used for the treatment of the laser transport was as follows. The laser path length was divided into $N$ separate zones, each of length $L_{z} \leqslant \alpha^{-1}$. Either one (for a single pass amplifier configuration) or two (for the simulation of a laser resonator) spatially dependent counter propagating fluxes $\phi^{+}$ans $\phi^{-}$were allowed. The quantities tracked in time were the zonal averages of these fluxes

$$
\phi_{n}^{ \pm}(t)=\int_{n L_{z}}^{(n+1) L_{z}} \phi^{ \pm}(x, t) d x / L_{z}
$$

and the similarly defined averages of the concentrations of all the gas components. Inside each zone $\phi^{+}\left(\phi^{-}\right)$was assumed to have a profile proportional to $\exp \left(\boldsymbol{\alpha}_{n} x\right)\left(\exp \left(\boldsymbol{\alpha}_{n}\left(L_{z}-x\right)\right)\right)$, where $\alpha_{n}$ was the gain coefficient determined from the average particle concentrations. The time dependence of the average fluxes was determined by the equations:

$$
\frac{1}{c} \frac{d}{d t} \phi_{n}^{ \pm}=\alpha_{n} \phi_{n}^{ \pm}-\phi_{\text {out }, n}^{ \pm}+\phi_{i n, n}^{ \pm}
$$


77

where

$$
\phi_{\text {out }, n}^{ \pm}=\frac{\alpha_{n} \exp \left(\alpha_{n} l_{z}\right)}{\left.\left(\exp r_{n} l_{z}\right)-1\right)} \phi_{n}^{ \pm}
$$

and for $2 \leqslant n \leqslant N, \phi_{i n, n}^{+}=\phi_{\text {out }, n-1}^{+}$. The value of $\phi_{i n, 1}^{+}$was set is y considering the boundary conditions. When modelling a laser resonator, $\phi_{i n, 1}^{+}$was set equal to $R \cdot \phi_{\text {out }, 1}^{-}$, where $R$ was the mirror reflectivity; for a single pass amplifier, $\phi_{i n, 1}^{+}=c \cdot \phi_{\text {input }} / l_{2}$, and $\phi_{n}^{-}=0$. The values of $\phi_{i n, n}^{-}$were defined in a similar manner. The effect of the laser photons on the particle concentrations was determined by assuming that the relevant species were created or destroyed at a rate depedent on the total average photon flux $\phi_{n}^{+}+\phi_{n}^{-}$.

Before this approximation was included in the model of the krypton fluoride laser kinetics, it was tested by applying it in some simple kinetic situations which could be solved analytically. First, we calculated the steady state output flux $\phi_{f}$ for the system of equations:

$$
\frac{\partial}{\partial \mathrm{t}} \mathrm{n}=\varsigma-\mathrm{n} / \tau-\mathrm{n} \sigma \phi ; \frac{1}{\mathrm{c}} \frac{\partial}{\partial \mathrm{t}} \phi=-\frac{\partial}{\partial \mathrm{x}} \phi+n \sigma \phi ; \quad 0 \leqslant x \leqslant L
$$

with $\phi(0, t)=\phi_{i}$. The analytic solution in this case is given 
implicitly by

$$
\ln \left(r_{f} / r_{i}\right)+\left(r_{i}-r_{i}\right)=g_{0}
$$

where $g_{0}=S \sigma \tau L$ is the logarithm of the stwali signal gain, and the "r's" are the ratios of the relevant fluxes to the saturation flux

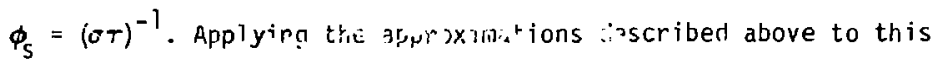
case using only on, wite yis.s

$$
r_{f}=r_{i} \exp \left(g_{o} /(7+r)\right)
$$

where $r$ is determined from the equation

$$
\frac{1}{\left(1+\frac{q_{0}}{r} \cdot r\right.} \frac{1}{\left(\exp \left(g_{0} /(1+r)-1\right)\right.}=r_{i}
$$

For $g_{0}$ ranging from .1 to 100 , and $r$, from .1 to 10 the values of $r_{f}$ obtained from equations 12 and 13 were ident:cal to the limits of computation, and are presented in Table IV.

The second test cait chosen corresponded to the kinetic equations

$$
\frac{\partial}{\partial t} n=-c n \phi ; \frac{1}{c} \frac{\partial}{\partial t} \phi=-\frac{\partial}{\partial x} \phi+n \sigma \phi ; 0 \leq x \leq L
$$

with the boundary conditiens $n(x, 0)=n_{0}, \phi(x>0,0)=0$, and $\phi(0, t)=\phi_{i}$. In this case ti.e output flux can be found analytically as function of time; defining $\tau=c t / L$ and $r_{f}=\phi(L, t) / c n_{0}$ (similarly 
for $r_{j}$ ) and with $g_{0}=n_{0} \sigma L$, we have

$$
r_{f}(\tau)=\frac{r_{0} \exp \left(r_{0} g_{0}(\tau-1)\right)}{\exp \left(r_{0} g_{0}(\tau-1)-\exp \left(-g_{0}\right)\right.}
$$

The agreement between this analytic solution and the result obtained by applying the approximate photon transport method described above (again using only ane zone) is shown in Figure $5, r$ the case $g_{0}=1$, $r_{0}=.01$ (assuming a I cm. zone length, this choice of parameters would imply an initial KrF* upper state density of $3.8 \times 10^{15} \mathrm{~cm}^{-3}$, a stored energy density of $3 \mathrm{~J} / \mathrm{liter}$, and an incident $\mathrm{flus}$ of $1 \mathrm{MW} / \mathrm{cm}^{2}$ ). Further analysis indicates that the condition for the applicabilty if our transport approximation is that the zone length be less than $\alpha(d \alpha / d x)^{-1}$, the scale length for variations in the gain coefficient, a much weaker constraint than that imposed when Eq. 7 is integrated directly, and one which can be readily satisfied in all the cases considered.

In addition to tracking the laser flux, we must consider the amplification of the spontaneous emission in directions other than the laser axis. Such processes may drastically reduce the laser performance in cases where the transverse gain is greater than 1 , and would require at least a two-dimensional calculation for an accurate representation. In this work we have treated only those geometries with small transverse gains, in which case we have assumed that in each zone the den.ity of the "stimulated-spontaneous" emission $P_{S}(\nu)$ 
grows according to the equation

$$
\frac{l}{c} \frac{d}{d t} \rho_{S}(\nu)=S(\nu)+\alpha(\nu) \rho_{S}(\nu)-\rho_{S}(\nu) / R
$$

where $S(\nu)$ is the local source term due to radiative decay of $\mathrm{KrF}^{*}$, $\alpha(\nu)$ the local gain coefficient at the frequency $\nu$, and $R$ is the average path length that a spontaneously emitted photon emitted in the zone will travel before hitting a laser wall

With this approximation for the solution to Eq. 7, the laser kinetics reduces to a system of coupled nonlinear differential equations in time, requiring only numerical integration for the completion of the kinetic model. Such systems are generally solved using a variable time-step stiff predictor-corrector technique such as those describes by Gear. ${ }^{115}$; however application of these methods to the laser kinetics leads to an instabilty (in which the photon fluxes become and grow increasingly negative due to "stimulated absorption") which can only be avoided at prohibitive computational cost. For this reason we decided to use instead of'the standard methods a four-point Runge-Kutta scheine with the time step being set by requiring $\dot{y} \Delta t \leq$ $.05 y$ for $\dot{y}>0$ (and $\dot{y} \Delta t \leq-.5 y$ for $\dot{y}<0$ ), where $y$ and $\dot{y}$ are respectively the values and time derivatives of any of the quantities integrated.

Several checks were written into the integration scheme to catch any errors in inputting the form of the various reactions. The local electrci density was calculated by integrating its kinetic equation, rather than by setting it equal to the net heavy particle positive 
charge density. The density of fluorencence photons from the various excited states, as well as the gas and electron thermal energy densities were also followed via integration. This allowed verification of the computer input by requiring that the net charge density, net atom densities, and total energy be conserved to the round-off accuracy of the computer (typically better that 1 part in $\left.10^{12}\right)$.

To avoid computational waste, several computer codes are written to implement the kinetics model depending on the physical characteristics of the problem under consideration. In cases where the initial gas mixture was restricted (e.g. containing only $\mathrm{kr}$ and $F_{2}$ ) all reactions requiring the forbidden gas components were removed. For those systems where the gas temperature remained essentially consiant any dependence on $\mathrm{T}_{g}$ was removed and all rate constants were set equal to their room temperature values. When the effect of the spontaneous KrF* emission was determined to be negligb?it spectrum was compressed to a delta function at the peak wavelength. Finally, for each set of conditions separate programs were written to model the behavior of single pass amplifier and laser resonators.

The code to model the single pass behavior of electron beam pumped mixiures or krypton, argon, helium and fluorine, CELLKIN, is presented in the microfiche at the end of this thesis. It assumes that the transverse gain is negligible, and that the gas temperature rise caused by the electron beam deposition is small. It may be 
compiled for up to a maximum of 20 zones in the laser region, and outputs both the spatial and temporal dependence of the gas parameters and amplifier output.

r.ELLKIN was used to model the behavior of electron beam pumped mixtures in the MEG I gas cell at LLNL, which will be described in detail in the following chapter. For the current densities $\quad \leqslant 200$ amp $/ \mathrm{cm}^{2}$ ) and gas pressures of interest here, the product of the gain coefficient and the cell length was $\leqslant 1$, allowing a single zone treatment of the photon transport.

The time dependence of the $r_{r} \mathrm{~F}^{\star}$ and $\mathrm{Kr}_{2} \mathrm{~F}^{*}$ fluorescence signals from a 1500 Torr Ar, 100 Torr $\mathrm{Kr}, 5.00$ Torr $\mathrm{F}_{2}$ gas mixture pumped with a $50 \mathrm{nsec}, 600 \mathrm{krl}$ electron beam at an average current density of $33 \mathrm{Amp} / \mathrm{cm}^{2}$ is pres, ted in Figure 6 . Both the results determined from experiulent 116 and those calculated by the model are shown, all normalized to their peak values. In Figure 7 we show the corresponding results for a 11,600 Torr He, 100 Torr $\mathrm{Kr}, 5.00 \mathrm{Torr}$ $\mathrm{F}_{2}$ mixture pumped with a similar e-beam current pulse. Although a direct comparison of the model and experiment could not be made because the fluorescence detection efficiencies were not known, it is relevant to note that the conversion ratios, defined as the peak experimental $\mathrm{KrF}^{*}$ signal divider by the peak model $\mathrm{KrF}^{*}$ signal, agree to within $20 \%$ for the argon- anc hel ium-buffered mixtures. A similar result holds for the $\mathrm{Kr}_{2} \mathrm{~F}^{\star}$ ratios. 
The model and experimental resuit.s differ slightly both during and after the current pulse. The disagreement between the experimental and model dimer signals at the end of the current pulse is probably due to a failure in modelling the time-dependent destruction (presumably by electrons) of $\mathrm{KrF}^{*}$. The longer decay time for the trimer predicted by the mode! than obtained in the experiment might be caused by the neglect of rare gas quenching of $\mathrm{Kr}_{2} \mathrm{~F}^{*}$ in the model. Both of these points will be discussed in the next chapter.

In Figure 8 we show the spectral dependence (at the end of the current pulse) of the absorption by a $1500 \mathrm{Torr}$ Ar, $100 \mathrm{Torr} \mathrm{Kr}, 5.00$ Torr $F_{2}$ gas pumped with a $50 \mathrm{nsec} ., 600 \mathrm{loV}$ electron beam at an average current density of $\approx 140 \mathrm{Amp} / \mathrm{Cl}$, experimental data is that of Powell. 116 The energy deposition into the gas is assumed to be twice the value calulated for a single pass, in line with pressure rise measurements and numerical calculations of the electron beam transport. 117 While the model appears to accurately estimate the gain at $248 \mathrm{~nm}$. , it fails to predict the currently unidentified sharp absorption feature at $\simeq 220 \mathrm{~nm}$. In addition the calculation underestimates the broad-band absorption centered at $\simeq 320 \mathrm{~nm}$., which could be the result of overestimating the effects of electron collisional quenching of the $\mathrm{Kr}_{2} \mathrm{~F}^{*}+$ rimer, to be discussed in Chapter V.

The breakdown by absorber of the absorption spectrum predicted by the model is illustrated in Fig. 9. The continuum absorption at long 
wavelengths is primarily from $\mathrm{Kr}_{2} \mathrm{~F}^{*}$. At the laser wavelength, the saturable absorption due to $\mathrm{Kr}_{2} \mathrm{~F}^{*}$ is approximately one-third of the total. The remair:der of the loss is predicted to be nonsaturable, and mainly due to $\mathrm{KrF}^{*}$ precursors (e.g. Ar $4 \mathrm{p}, \mathrm{Kr} 2+$, etc.), with a small portion $(\approx 10 \%)$ from absorption by $\mathrm{Ar}_{2} \mathrm{~F}^{\star}$.

We conclude ty determining the effect that this breakdown of the medium absorption would have on the performance of a $\mathrm{KrF}^{*}$ laser operating using this gas mixture and current density. At the rulse lengths of interest ( $>10 \mathrm{~ns}$. ), th gas kinetics reaches a quasi-steady state, in which case the analysis of Chapter I dealing with the consequences of medium absorption is applicatsle. Specifically, institing the gain and loss crefficients obtained above into Eq. 11 of that chapter results in a prediction of less than $50 \%$ for the maximum local extraction efficiency. Due to spatial variations in the laser intensity one would expect the average extraction effic'ency in the laser to be even lower. In Chapter VI we will consider this problem in detail arid present the results obtained under actual laser operation. 


\section{Table I \\ Particle Concentrations Tracked \\ In Laser Kineiics Model}

\begin{tabular}{|c|c|c|c|}
\hline State & Energy (ev) & State & Energy $(\mathrm{eV})$ \\
\hline Ar & 0.0 & $\mathrm{Kr}$ & 0.0 \\
\hline $\mathrm{Ar}^{+}$ & 15.76 & $\mathrm{Kr}^{+}$ & 14.00 \\
\hline $\mathrm{Ar}_{2}^{+}$ & 14.50 & $\mathrm{Kr}_{2}^{+}$ & 12.90 \\
\hline$A r^{\star}(\operatorname{Ar} 4 s)$ & 11.76 & $K r *(K r 5 s)$ & 9.98 \\
\hline$A r \star \star(A r 4 p)$ & 13.00 & $K r \star \star(K r \quad 5 p)$ & 11.50 \\
\hline $\operatorname{Ar}_{2} *\left({ }^{3} \Sigma\right)$ & 11.06 & $\mathrm{Kr}_{2}{ }^{*}\left({ }^{3} \Sigma\right)$ & 9.08 \\
\hline $\operatorname{Ar}_{2}{ }^{\star}\left({ }^{l} \Sigma\right)$ & 11.135 & $\mathrm{Kr}_{2} *\left({ }^{l} \Sigma\right)$ & 9.20 \\
\hline ArF* & 7.24 & $\mathrm{KrF} *$ & 5.90 \\
\hline $\mathrm{Ar}_{2} \mathrm{~F}^{\star}$ & 6.10 & $\mathrm{Kr}_{2} \mathrm{~F}^{\star}$ & 4.40 \\
\hline ArkrF* & 5.20 & & \\
\hline He & 0.0 & He $2^{3} \mathrm{~s}$ & 19.82 \\
\hline $\mathrm{He}^{+}$ & 24.59 & He $2^{l} \mathrm{~S}$ & 20.615 \\
\hline $\mathrm{He}_{2}^{+}$ & 22.23 & He $2^{3} p$ & 20.96 \\
\hline $\mathrm{He}_{3}^{+}$ & 22.06 & $\operatorname{He} 2^{7} p$ & 21.22 \\
\hline $\mathrm{He}_{2}{ }^{\star}$ & 17.97 & & \\
\hline $\mathrm{F}_{2}$ & 0.0 & $F *(3 s)$ & 13.57 \\
\hline$F$ & 0.80 & $F^{\star}(3 p)$ & 15.30 \\
\hline$F^{-}$ & -2.66 & $\mathrm{~F}_{2}{ }^{\star}$ & 11.05 \\
\hline electrons & $\cdots+$ & & \\
\hline
\end{tabular}


Table II

Reaction Kinetics

Reaction

$$
\mathrm{k}_{\mathrm{R}}\left(\mathrm{cm}^{3} / \mathrm{s} \text { or } \mathrm{cm}^{6} / \mathrm{s}\right)
$$

Source

Krypton-Krypton Reactions

$$
\begin{aligned}
& \mathrm{Kr}^{+}+\mathrm{Kr}+\mathrm{M}-\mathrm{Kr}_{2}^{+}+\mathrm{M} \\
& R \times 2.5 \times 10^{-37} \mathrm{~A} \text { (a) } \\
& K r^{\star \star}+K r+M \rightarrow-K r^{\star}+K r+M \\
& R \times 5.4 \times 10^{-32} A \text { (b) } \\
& \mathrm{Kr} r^{\star}+\mathrm{Kr}+\mathrm{M}-\mathrm{Kr}_{2}{ }^{\star}+\mathrm{M} \\
& R \times 2.6 \times 10^{-32} \mathrm{~A} \\
& 1.5 \times 10^{-10} \mathrm{~A} \\
& 2 \mathrm{Kr}_{2}{ }^{\star}-\mathrm{Kr}_{2}^{+}+2 \mathrm{Kr}+\mathrm{e}^{-} \\
& 1.0 \times 10^{-10} \mathrm{~A} \\
& \mathrm{Kr} * \star+\mathrm{Kr}+\mathrm{M} \rightarrow \mathrm{Kr}{ }_{2}^{\star}+\mathrm{M} \\
& R \times 2.6 \times 10^{-32} \mathrm{~A} \\
& 3.0 \times 10^{-11} \mathrm{~A} \quad \text { (d) } \\
& 2 \mathrm{Kr}^{\star}-\mathrm{Kr}^{+}+\mathrm{Kr}+\mathrm{e}^{-} \\
& K r^{\star \star}+K r-K r *+K r \\
& \mathrm{Kr}_{2}{ }^{\star}\left({ }^{\mathrm{l}} \Sigma\right)+\mathrm{M} \\
& -\mathrm{Kr}_{2} \star\left(^{3} \Sigma\right)+M \\
& 2.8 \times 10^{-13} \mathrm{~A} \quad \text { (e) }
\end{aligned}
$$

Krypton-Argon Reactions

$$
\begin{array}{ll}
\mathrm{Ar}_{2}^{+}+\mathrm{Kr}-\mathrm{Kr}^{+}+2 \mathrm{Ar} & 7.5 \times 10^{-10} \mathrm{~A} \\
\mathrm{Ar}^{\star}+\mathrm{Kr}-\mathrm{Kr}{ }^{\star}+\mathrm{Ar} & 6.2 \times 10^{-12} \mathrm{~A} \\
\mathrm{Ar}_{2}{ }^{\star}+\mathrm{Kr}-\mathrm{Kr}^{\star}+2 \mathrm{Ar} & 1.0 \times 10^{-10} \mathrm{~A}
\end{array}
$$

Note $A=\left(T_{g} / 300^{\circ} \mathrm{K}\right)^{.5}$
(a) $M=H e, K=.25 ; M=A r, k=1.3 ; M=k r, R=1$.
(b) $M=H e, R=4$; $M=A r, R=1.3 ; M=k r, R=1$.
(c) Rate constant equals that for the $k r^{\star}$ reaction.
(d) Rate constant equals that for the similar Ar reaction.
(e) Rate constant given is for the forward reaction, that for the reverse reaction is calculated from vetailed balance. 
Tabie II (contirued)

$$
\text { Reaction Kinetics: }
$$

Reaction

$$
k_{R}\left(\mathrm{~cm}^{3} / \mathrm{s} \text { or } \mathrm{cm}^{6} / \mathrm{s}\right)
$$

Source

Argon-Argon Reactions

$$
\begin{aligned}
& \mathrm{Ar}^{+}+\mathrm{Ar}+\mathrm{M}-\mathrm{Ar}_{2}^{+}+\mathrm{M} \\
& R \times 2.5 \times 10^{-31} A \quad \text { (a) } \\
& A r^{\star} \star A r+M-A r^{\star}+A r+M \\
& R \times 1.0 \times 10^{-31} \mathrm{~A} \\
& A r^{\star}+A r+M \rightarrow A r_{2}{ }^{\star}+M \\
& \mathrm{Rx}] .1 \times 10^{-32} \mathrm{~A} \\
& 2 A r *-A r^{+}+A r+e^{-} \\
& 1.0 \times 10^{-10} \mathrm{~A} \\
& 2 \mathrm{Ar}_{2}{ }^{\star}-\mathrm{Ar}_{2}^{+}+2 \mathrm{Ar}+\mathrm{e}^{-} \\
& 1.0 \times 10^{-10_{A}} \\
& \operatorname{Ar}_{2} *(' \Sigma)+M \\
& -\operatorname{Ar}_{2} *\left(^{3} \Sigma\right)+M \\
& 1.5 \times 10^{-13} \mathrm{~A}
\end{aligned}
$$

Helium-Krypton Reactions

$$
\begin{array}{lr}
\mathrm{He}_{2}^{+}+\mathrm{Kr} \rightarrow \mathrm{Kr}^{+}+2 \mathrm{He} & 2.3 \times 10^{-11_{A}} \\
\mathrm{He} 2^{3} \mathrm{~S}+\mathrm{Kr}-\mathrm{e}^{-} & \text {calculated from } \\
+\left(\mathrm{HeKr}{ }^{+}\right. \text {or He+Kr } & \text { cross section } \\
\mathrm{HeKr}^{+}+\mathrm{Kr}-\mathrm{Kr}^{+}+\mathrm{He}+\mathrm{Kr} & 1.1 \times 10^{-8}(\mathrm{kT} / \mathrm{geV}) \cdot 5
\end{array}
$$

Note $A=\left(T_{g} / 300^{\circ} \mathrm{K}\right)^{.5}$

(a) $M=H e, R=.3 ; M=A r, R=1 . ; M=K r, R=.8$

(b) $M=H e, R=2.5 ; M=A r, R=1, ; M=k r, R=.8$

(c) Rate constant given is for the forward reaction, that for the reverse reaction is calculated from detailed balance.

(d) Rate constant is the integral of the cross section over a Maxwellian. See the microfiche listing of the kinetics. 
Table II (continued)

\section{Reaction Kinetics}

Reaction

$k_{R}\left(\mathrm{~cm}^{3} / \mathrm{s}\right.$ or $\left.\mathrm{cm}^{6} / \mathrm{s}\right)$

Source

Helium-Krypton Reactions (continued)

$\mathrm{HeKr}^{+}+\mathrm{Kr}-\mathrm{Kr}_{2}^{+}+\mathrm{He}$

$3.6 \times 10^{-10}\left(\mathrm{kT} \mathrm{g}^{/ l \mathrm{lV})}\right)^{.5}$

He $2^{3} \mathrm{~S}+\mathrm{Kr}+\mathrm{M}-$

$$
\mathrm{e}^{-}+\mathrm{Kr}^{+}+\mathrm{He}+\mathrm{M} \quad 2.2 \times 10^{-30} \mathrm{~A} \quad \text { (a) }
$$

He $2^{1} \mathrm{~S}+\mathrm{Kr} \rightarrow \mathrm{e}^{-}$

$$
\begin{array}{ll}
+.2 \mathrm{HeKr}^{+}+.8\left(\mathrm{He}+\mathrm{Kr}^{+}\right) & 4.8 \times 10^{-10} \\
\mathrm{He}_{2}^{+}+\mathrm{Kr}+\mathrm{M} \rightarrow \mathrm{Kr}^{+}+2 \mathrm{He}+\mathrm{M} & 2.4 \times 10^{-29} \mathrm{~A}^{-1.5} \quad \text { (a) } \\
\mathrm{He}_{2}{ }^{\star}+\mathrm{Kr} \rightarrow-\mathrm{Kr}^{+}+2 \mathrm{He}+\mathrm{e}^{-} & 3.1 \times 10^{-10} \mathrm{~A} \quad \text { (a) }
\end{array}
$$

Hel ium-Argon Reactions

$$
\mathrm{He}_{2}^{+}+\mathrm{Ar} \rightarrow \mathrm{Ar}^{+}+2 \mathrm{He}
$$

He $2^{3} s+A r-e^{-}$

$$
+\left(\mathrm{HeAr}^{+} \text {or } \mathrm{He}+\mathrm{Ar}^{+}\right)
$$$$
\mathrm{HeAr}{ }^{+}+\mathrm{Ar}-\mathrm{Ar}^{+}+\mathrm{He}+\mathrm{Ar}
$$$$
\mathrm{HeAr}{ }^{+}+\mathrm{Ar}-\mathrm{Ar}_{2}^{+}+\mathrm{He}
$$$$
\text { He } 2^{3} S+A r+M-
$$

$$
\begin{array}{cl}
\mathrm{e}^{-}+\mathrm{Ar}{ }^{+}+\mathrm{He}+\mathrm{M} & 2.2 \times 10^{-30} \mathrm{~A} \\
\mathrm{He}_{2}{ }^{\star \mathrm{AH}}-\mathrm{Ar}^{+}+{ }_{\mathrm{CHe}}+\mathrm{e}^{-} & 3.1 \times 10^{-10} \mathrm{~A}
\end{array}
$$$$
2.3 \times 10^{-10} \mathrm{~A}
$$$$
\text { calculated from }
$$$$
\text { cross section (b) }
$$$$
1.1 \times 10^{-8}(\mathrm{kT} / \mathrm{geV}) \cdot 5
$$$$
1.1 \times 10^{-9}(\mathrm{kT} / \mathrm{geV}) .5
$$

Note $A=\left(T_{g} / 300^{\circ} \mathrm{K}\right) \cdot 5$

(a) Rate constant equals that for the similar Ar reaction.

(b) Both the rate constant and yield for this reaction are temperature dependent. See the microfiche listing. 
Table II (continued)

\section{Reaction Kinetics}

Reaction

$$
\mathrm{r}_{\mathrm{R}}\left(\mathrm{cm}^{3} / \mathrm{s} \text { or } \mathrm{cm}^{6} / \mathrm{s}\right)
$$

Source

Helium-Argon Reactions (continued)

He 2 ' $S+A r-e^{-}$

$$
+.18 \mathrm{HeAr}^{+}+.82\left(\mathrm{He}+\mathrm{Ar}^{+} ; \quad 2.6 \times 10^{.10}\right.
$$

$\mathrm{He}_{2}^{+}+\mathrm{Ar}+\mathrm{M}-\mathrm{Ar}{ }^{+}+2 \mathrm{He}+\mathrm{M}$

$2.4 \times 10^{-29} A^{-1.5}$

He $2^{1} \mathrm{P}+\mathrm{Ar}=\mathrm{e}^{-}+\mathrm{Ar}^{+}+\mathrm{He}$

$1.7 \times 10^{-10} \mathrm{~A}$

Helium-Helium Reactions

$$
\begin{aligned}
& \mathrm{He}^{+}+\mathrm{He}+\mathrm{M}-\mathrm{He}_{2}^{+} \mathrm{M} \\
& 2 \mathrm{He}{ }^{3} \mathrm{~S}-.7 \mathrm{He}_{2}^{+}
\end{aligned}
$$$$
9.0 \times 10^{-32} / \mathrm{A}
$$

$$
+.3\left(\mathrm{He}+\mathrm{He}^{+}\right)+\mathrm{e}^{-} \quad 9.0 \times 10^{-10}
$$

$\mathrm{He} 2^{3} \mathrm{~S}+2 \mathrm{He}-\mathrm{He}_{2}{ }^{\star}+\mathrm{He}$

$$
8.3 \times 10^{-31}
$$

$$
x \exp \left(-.15\left(17.5+1 \mathrm{ev} / \mathrm{kT} \mathrm{T}_{\mathrm{g}}\right)\right)
$$

$\mathrm{He} 2^{3} \mathrm{~S}^{3} \mathrm{He}_{2}$ * $-\mathrm{e}^{-}+.85 \mathrm{He}_{2}^{+}$

$$
+.15\left(\mathrm{He}^{+} \mathrm{He}^{+}\right)+\mathrm{He} \quad 1.2 \times 10^{-9}
$$

$$
2 \mathrm{He}_{2}{ }^{*}-\mathrm{e}^{-}+.85 \mathrm{He}_{2}^{+}
$$

$$
+.15\left(\mathrm{He}+\mathrm{He}^{+}\right)+2 \mathrm{He}
$$

$$
1.2 \times 10^{-9}
$$

He 2 ? $\mathrm{P}+\mathrm{He}-2 \mathrm{He}$

$9.3 \times 10^{-13} \mathrm{~A}$

He 2 P $+2 \mathrm{He}-\mathrm{He}_{2}{ }^{\star}+\mathrm{He}$

$3.1 \times 10^{-31} \mathrm{~A}$

He $2^{1} \mathrm{~S}+\mathrm{He}-2 \mathrm{He}$

$6.8 \times 10^{-15} \mathrm{~A}$

He $2^{1} \mathrm{~S}+2 \mathrm{He}-\mathrm{He}_{2}{ }^{\star}+\mathrm{He}$

$1.3 \times 10^{-33} \mathrm{~A}$

$$
\text { Note } A=\left(T_{g} / 300^{\circ} \mathrm{K}\right)^{.5}
$$


Table II (continued)

Reaction Kinetics

Reaction

$k_{R}\left(\mathrm{~cm}^{3} / \mathrm{s}\right.$ or $\left.\mathrm{cm}^{6} / \mathrm{s}\right)$

Source

Electron elastic collisions

$e^{-}+e^{-}+M$

calculated from momentum

transfer cross sections

$(6,52,53)$

$e^{-}+10 n-e^{-}+10 n$

Spitzer's result used for

energy exchange

Electron Attachment and Recombination

$$
\begin{aligned}
& e^{-}+F_{2}-F^{-}+F \\
& K\left(1+2\left(R+R^{2}\right)\right) \quad(a) \\
& e^{-}+k r_{2}^{+}-k r^{\star \star}+k r \\
& 3.9 \times 10^{-7}\left(1 \mathrm{eV} / \mathrm{kT}_{\mathrm{e}}\right)^{.5} \\
& x\left(1-\exp \left(-.022 \mathrm{eV} / \mathrm{k} T_{g}\right)\right) \\
& e^{-}+A r_{2}^{+}-A r^{\star \star}+A r \\
& 7.8 \times 10^{-7}\left(\mathrm{leV} / \mathrm{kT}_{\mathrm{e}}\right)^{.67} \\
& x\left(1-\exp \left(-.037 \mathrm{eV} / \mathrm{kT}_{g}\right)\right) \\
& 2 \mathrm{e}^{-}+\mathrm{He}_{2}^{+}-\mathrm{e}^{-} \\
& +.7 \mathrm{He} 2^{3} \mathrm{~S}+1.3 \mathrm{He} \\
& 4.0 \times 10^{-20}\left(T_{g} / T_{e}\right)^{4} \\
& \mathrm{e}^{-}+\mathrm{He}_{3}-.7 \mathrm{He} 2^{3} \mathrm{~S}
\end{aligned}
$$$$
+2.3 \mathrm{He}+\mathrm{e}^{-}
$$$$
4.6 \times 10^{-6}\left(T_{g} / T e^{11.2}\right.
$$

Note $A=\left(T g / 300^{\circ} \mathrm{K}\right) \cdot 5$

(a) $R=\exp \left(-.1146 \mathrm{ev} / \mathrm{kT}_{g}\right)$ and if $k T_{e}<1 / 3, k=1.0 \times 10^{-8}$;

$$
\begin{aligned}
& \text { if } 1 / 3 \leq k T_{e} \leq .8, k=1.0 \times 10^{-9}\left(3.3+30.4\left(.8-k_{\mathrm{e}}\right)^{2}\right) \text {; } \\
& \text { if } k T_{e}>.8, k=3.0 \times 10^{-9} \text {. }
\end{aligned}
$$

(b) $\mathrm{He}_{3}^{+}$is assumed in equilibrium wich $\mathrm{He}_{2}^{+}$. 
Table II (continued)

Reaction Kiretics

Electron Collisional Ionization

$$
\begin{aligned}
& \text { Reaction } \\
& k_{R}\left(\mathrm{~cm}^{3} / \mathrm{s} \text { or } \mathrm{cm}^{6} / \mathrm{s}\right) \\
& \mathrm{e}^{-\mathrm{K} \mathrm{Kr}_{2}{ }^{\star}\left({ }^{3} \Sigma\right)-} \\
& 7.7 \times 10^{-7} \mathrm{~B}^{.5} \\
& \mathrm{Kr}_{2}^{+}+2 \mathrm{e}^{-} \\
& x \exp (-B) /(.97+B) \\
& e^{-}+k r_{2}{ }^{*}\left({ }^{1} \Sigma\right) \rightarrow \\
& 7.7 \times 10^{-7} \mathrm{~B}^{.5} \\
& \mathrm{Kr}_{2}^{+}+2 \mathrm{e}^{-} \\
& \times \exp (-E) /(.97+B) \\
& e^{-}+A r_{2} \star\left(^{3} \Sigma\right) \rightarrow \\
& 9.0 \times 10^{-7} B^{.5} \\
& \mathrm{Ar}_{2}^{+}+2 \mathrm{e}^{-} \\
& x \exp (-B) /(.97+B) \\
& \mathrm{e}^{-}+A r_{2} *\left({ }^{1} \Sigma\right)- \\
& 9.0 \times 10^{-7} B .5 \\
& \mathrm{Ar}_{2}^{+}+2 \mathrm{e}^{-} \\
& \times \exp (-B) /(.97+B) \\
& \mathrm{e}^{-}+\mathrm{kr} \rightarrow-\mathrm{Kr}^{+}+2 \mathrm{e}^{-} \\
& e^{-}+K r^{\star}-K r^{+}+2 e^{-} \\
& \mathrm{e}^{-}+\mathrm{Kr} \star \star-K r^{+}+2 \mathrm{e}^{-} \\
& \mathrm{e}^{-}+\mathrm{Ar}-\mathrm{Ar}+2 \mathrm{e}^{-} \\
& e^{-}+A r^{*}-A r^{+}+2 e^{-} \\
& e^{-}+A r^{\star \star}-A r^{+}+2 e^{-} \\
& \mathrm{e}^{-}+\mathrm{He}-\mathrm{He}^{+}+2 \mathrm{e}^{-} \\
& \mathrm{e}^{-}+\mathrm{He} 2^{3} \mathrm{~S} \rightarrow \mathrm{He}^{+}+2 \mathrm{e}^{-}
\end{aligned}
$$

Source

$(\mathrm{a}) \mathrm{B}=\left(3.82 \mathrm{eV} / \mathrm{kT} \mathrm{T}_{\mathrm{e}}\right) \quad(\mathrm{b}) \mathrm{B}=(3.70 \mathrm{eV} / \mathrm{kT} \mathrm{e}) \quad(\mathrm{c}) \mathrm{B}=\left(3.44 \mathrm{eV} / \mathrm{kT} \mathrm{T}_{\mathrm{e}}\right)$ (d) $B=\left(3.365 e V / k T_{e}\right)$

(d) Rate constant is the integral of the cross section over a Maxwellian. See the microfiche listing of the kinetics. 
Table II (continued)

Reaction Kinetics

Electron Collisiorid Excita ,ion and Superelastic Collisions

Reacticn

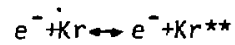

$\mathrm{e}^{-}+\mathrm{K} r^{\star} \leftrightarrow \mathrm{e}^{-}+\mathrm{Kr} r^{\star \star}$

$\mathrm{e}^{-}+\mathrm{Kr} \leftrightarrow \mathrm{e}^{-}+\mathrm{Kr} *$

$\mathrm{e}^{-}+\mathrm{Ar} \rightarrow \mathrm{e}^{-}+\mathrm{Ar} \star \star$

$\mathrm{e}^{-}+A r^{\star} \leftrightarrow \mathrm{e}^{-}+A r^{\star \star}$

$\mathrm{e}^{-}+\mathrm{Ar} \leftrightarrow \mathrm{e}^{-}+\mathrm{A} r^{\star}$

$\mathrm{e}^{-}+\mathrm{He} \rightarrow \mathrm{e}^{-}+\mathrm{He} 2^{3} \mathrm{~s}$

$\mathrm{e}^{-}+\mathrm{He} \leftrightarrow \mathrm{e}^{-}+\mathrm{He} 2^{\mathrm{l}} \mathrm{s}$

$\mathrm{e}^{-}+\mathrm{He} \leftrightarrow \mathrm{e}^{-}+\mathrm{He} 2^{3} \mathrm{p}$

$\mathrm{e}^{-}+\mathrm{He} \leftrightarrow \mathrm{e}^{-}+\mathrm{He} 2^{1} \mathrm{P}$

$\mathrm{e}^{-}+\mathrm{He} 2^{3} \mathrm{~S} \leftrightarrow \mathrm{e}^{-}+\mathrm{He} 2^{\mathrm{l}} \mathrm{S}$

$\mathrm{e}^{-}+\mathrm{He} 2^{3} \mathrm{~S} \leftrightarrow \mathrm{e}^{-}+\mathrm{He} 2^{3} \mathrm{p}$

$\mathrm{e}^{-}+\mathrm{He} 2^{3} \mathrm{~s} \rightarrow \mathrm{e}^{-}+\mathrm{He} 2^{1} P$

$\mathrm{e}^{-}+\mathrm{He} 2^{1} \mathrm{~S} \leftrightarrow \mathrm{e}^{-}+\mathrm{He} 2^{3} \mathrm{p}$

$\mathrm{e}^{-}+$He $2^{l} \mathrm{~S} \leftrightarrow \mathrm{e}^{-}+$He $2^{l} \mathrm{P}$

$\mathrm{e}^{-}+\mathrm{He} 2^{3} \mathrm{p} \leftrightarrow \mathrm{e}^{-}+\mathrm{He} 2^{l} \mathrm{p}$
Source

$(75,76)$

(78-80,83-85)

$(78,80,86)$

$(78,80,83,84,86-88)$

Note The rate constants for the forward (endothermic) reactions are calculated by integrating the cross sections over a Maxwellian. The reverse rates are calculated from detailed balance. See the microfiche listing of the kinetics. 
Table II (continued)

Reaction Kinetics

Reaction

$\mathrm{k}_{\mathrm{R}}\left(\mathrm{cm}^{3} / \mathrm{s}\right.$ or $\left.\mathrm{cm}^{6} / \mathrm{s}\right)$

Source

Electron Collisional Excitation and Suparelastic Collisions (cont.)

$e^{-}+k r_{2}{ }^{\star}-e^{-}+2 k r$

$\mathrm{e}^{-}+k r_{5} \star(?)=$

$\mathrm{e}^{-}+\mathrm{Kr} \mathrm{r}_{2} *\left({ }^{3} \Sigma\right)$

$e^{-}+A r_{2}^{*}\left({ }^{1} \Sigma\right) \longrightarrow$

$e^{-}+\operatorname{Ar}_{2} *\left({ }^{3} \Sigma\right)$
$5.7 \times 10^{-9}\left(\mathrm{kT}_{\mathrm{e}} / \mathrm{leV}\right) \cdot 5$

(a)

$$
1.5 \times 10^{-7}\left(\mathrm{kT} \mathrm{e}^{/ 1 \mathrm{eV})^{.5}}\right.
$$

(a)

$7.5 \times 10^{-8}\left(\mathrm{kT} \mathrm{e}^{/ l \mathrm{eV})^{-5}}\right.$

Fluorine Reactions

$$
\begin{aligned}
& \mathrm{e}^{-}+\mathrm{F}_{2}-\mathrm{F}^{-\top}+\mathrm{F} \\
& \mathrm{He}^{+}+\mathrm{F}^{-}-\mathrm{He}+\mathrm{F} *(3 p) \\
& \mathrm{He}_{2}^{+}+\mathrm{F}^{-}- \\
& 2 \mathrm{He}+F *(3 p) \\
& e^{-*}+F_{2} \star-F^{-}+F
\end{aligned}
$$

$$
K\left(1+2\left(R+R^{2}\right)\right)
$$

see the microfiche listing (c) $(10,90,91)$

see the microfiche

$$
\text { listing (c) }
$$

$\left.K(]+2\left(R+R^{2}\right)\right)$

Note $A=\left(T_{g} / 300^{\circ} \mathrm{K}\right) \cdot 5$

(a) Rate constant given is for the forward reaction, that for the reverse reaction is calculated from detailed balance.

(b) See the fifth page of this Table.

(c) The reaction rates for atomic recombination were calculated according to the method of Flannery and Yang.

(d) Rate constant equals that for the similar $F_{2}$ reaction. 
Table II (continued)

\section{Reaction Kinetics}

Reaction

$$
k_{R}\left(\mathrm{~cm}^{3} / \mathrm{s} \text { or } \mathrm{cm}^{6} / \mathrm{s}\right)
$$

Source

Fluorine Reactions

$$
\begin{aligned}
& F \star(3 p)+F_{2}-F_{2}^{*}+F \\
& \text { 1. } \times 10^{-9} \mathrm{~A} \\
& 1 . \times 10^{-9} \mathrm{~A} \\
& \mathrm{~F}^{\star}(3 \mathrm{~s})+\mathrm{F}_{2}-\mathrm{F}_{2}{ }^{\star}+\mathrm{F} \\
& 1 . \times 10^{-9} \mathrm{~A} \\
& \text { He } 2^{1} \mathrm{~S}+\mathrm{F}_{2}-\mathrm{He}+\mathrm{F}+\mathrm{F} *(3 \mathrm{p}) \quad 1 . \times 10^{-9} \mathrm{~A} \\
& \text { He } 2^{3} \mathrm{P}+\mathrm{F}_{2} \rightarrow \mathrm{He}+\mathrm{F}+\mathrm{F} *(3 p) \quad 1 . \times 10^{-9} \mathrm{~A} \\
& \text { He } 2^{1} \mathrm{P}+\mathrm{F}_{2}-\mathrm{He}+\mathrm{F}+\mathrm{F}^{*}(3 \mathrm{p}) \quad 1 . \times 10^{-9} \mathrm{~A} \\
& \mathrm{He}_{2}{ }^{\star}+\mathrm{F}_{2}-2 \mathrm{He}+\mathrm{F}+\mathrm{F}^{\star}(3 \mathrm{p}) \quad \mathrm{I} \times 10^{-9} \mathrm{~A} \\
& K r+F \star(3 p)-K r^{+}+F+e^{-} \quad 1 . \times 10^{-10} A \\
& K r+F *(3 s)-K r \star \star * F \quad 1 . \times 10^{-10} \mathrm{~A} \\
& A r+F \star(3 p) \rightarrow A r^{\star \star+F} \quad 1 . \times 10^{-10} \mathrm{~A} \\
& A r+F *(3 s)-A r * F \quad 1 . F 10^{-10} A \\
& \underline{\text { KrF* Formation }} \\
& \mathrm{Kr}^{+}+\mathrm{F}^{-}-\mathrm{KrF}^{*} \quad \text { see the microfiche listing (b) }(10,90,91) \\
& \mathrm{Kr}_{2}^{+}+\mathrm{F}^{-}-\mathrm{KrF} \mathrm{F}^{\star}+\mathrm{Kr} \quad \text { see the microfiche listing (b) }(10,90,91)
\end{aligned}
$$

Note $A=\left(T_{g} / 300^{\circ} \mathrm{K}\right)^{.5}$

(a) These reactions were assumed to go at their gas kinetic rates.

(b) The reaction rates for atomic recombination were calculated according to the method of Flannery and Yang. 
Table II (continued)

Reaction Kinetics

Reaction

$$
k_{R}\left(\mathrm{~cm}^{3} / \mathrm{s} \text { or } \mathrm{cm}^{6} / \mathrm{s}\right)
$$

Source

KrF* Formation (continued)

$$
\begin{aligned}
& K r^{\star \star}+F_{2}-K r F^{\star}+F \\
& K r^{\star}+F_{2}-K r F^{\star}+F \\
& K r_{2}^{\star}+F_{2}-K r F^{\star}+K r+F \\
& K r+F_{2}^{\star}-K r F^{\star}+F
\end{aligned}
$$$$
7.6 \times 10^{-10} \mathrm{~A}
$$$$
7.6 \times 10^{-10} \mathrm{~A}
$$$$
3.0 \times 10^{-10} \mathrm{~A}
$$$$
7.6 \times 10^{-10} \mathrm{~A}
$$

ArF* Formation

$$
\begin{aligned}
& \mathrm{Ar}^{+}+\mathrm{F}^{-} \rightarrow \mathrm{ArF}^{\star} \\
& \mathrm{Ar}_{2}^{+}+\mathrm{F}^{-}-\mathrm{ArF} \mathrm{F}^{*} \mathrm{Ar} \\
& A r^{\star \star}+F_{2}-A r F^{\star}+F \\
& A r^{\star}+\mathrm{F}_{2}-\mathrm{ArF}^{\star}+\mathrm{F} \\
& \mathrm{Ar}_{2}:+\mathrm{F}_{2} \rightarrow \mathrm{ArF} \mathrm{F}^{\star}+\mathrm{Ar}+\mathrm{F} \\
& \mathrm{Ar}+\mathrm{F}_{2}{ }^{\star}-\mathrm{ArF} \mathrm{F}^{\star} \mathrm{F} \\
& \text { see the microfiche listing (b) }(10,90,91) \\
& 7.5 \times 10^{-10_{A}} \\
& 7.5 \times 10^{-10} \mathrm{~A} \\
& 7.5 \times 10^{-10} \mathrm{~A} \\
& 7.5 \times 10^{-10} \mathrm{~A} \\
& A r F^{\star}+F_{2}-A r+3 F \\
& 2.0 \times 10^{-9} \mathrm{~A} \\
& A r F^{*}+A r=2 A r+F \\
& 1.3 \times 10^{-11} \mathrm{~A} \\
& A r F^{*}+K r-K r F^{*}+A r \\
& 1.0 \times 10^{-9} \mathrm{~A}
\end{aligned}
$$$$
\text { see the microfiche listing (b) }(10,90,91)
$$

Note $A=\left(T_{g} / 300^{\circ} \mathrm{K}\right) \cdot 5$

(a) These reactions were assumed to go at the same rates as that for the excited rare gas and $F_{2}$.

(b) The reaction rates for atomic recombination were calculated according to the method of Flannery and Yang. 
Table II (continued)

Reaction Kinetics

Reaction

$k_{R}\left(\mathrm{~cm}^{3} / \mathrm{s}\right.$ or $\left.\mathrm{cm}^{6} / \mathrm{s}\right)$

Source

ArF* Quenching (continued)

$A F^{\star}+A r+M \rightarrow A r_{2} F^{\star}+M$

$R \times 3.2 \times 10^{-31} \mathrm{~A} \quad$ (a)

$(96,97)$

$\mathrm{ArF}{ }^{*}+\mathrm{e}^{-} \rightarrow \mathrm{Ar}+\mathrm{F}+\mathrm{e}^{-}$

$1 . \times 10^{-7}$

$\underline{\mathrm{KF}} \mathrm{F}^{\star}$ Quenching

$\mathrm{KrF}^{\star}+\mathrm{F}_{2}-\mathrm{Kr}+3 \mathrm{~F}$

$1.0 \times 10^{-9} \mathrm{~A}$

$\mathrm{KrF}^{\star}+\mathrm{Kr}-2 \mathrm{Kr}+\mathrm{F}$

$7.4 \times 10^{-13_{A}}$

$\mathrm{KrF}^{\star}+\mathrm{He}-\mathrm{Kr}+\mathrm{F}+\mathrm{He}$

$2 . \times 10^{-12} A$

Chapter VI

$\mathrm{KrF}^{\star}+\mathrm{Ar}+\mathrm{M} \rightarrow \mathrm{ArKrF}{ }^{*}+\mathrm{M}$

$R \times 7.6 \times 10^{-32} A$

$\mathrm{KrF} \mathrm{F}^{\star}+\mathrm{Kr}+\mathrm{M} \rightarrow \mathrm{Kr}_{2} \mathrm{~F}^{\star}+\mathrm{M}$

$R \times 5.8 \times 10^{-31} A \quad$ (c)

(99-101)

$\mathrm{KrF}^{\star}+\mathrm{e}^{-}-\mathrm{Kr}+\mathrm{F}+\mathrm{e}^{-}$

$2 . \times 10^{-7}$

$\mathrm{Kr}_{2} \mathrm{~F}^{\star}$ Quenching

$\mathrm{Kr}_{2} \mathrm{~F}^{\star}+\mathrm{F}_{2} \rightarrow-2 \mathrm{Kr}+3 \mathrm{~F}$

$1.6 \times 10^{-10} \mathrm{~A}$

Chapter $V$

$\mathrm{Kr}_{2} \mathrm{~F}^{\star}+\mathrm{Kr}-3 \mathrm{Kr}+\mathrm{F}$

$<10^{-13} \mathrm{~A}$

Chapter $V$

$\mathrm{Kr}_{2} \mathrm{~F}^{\star}+\mathrm{e}^{-}-2 \mathrm{Kr}+\mathrm{F}+\mathrm{e}^{-}$

$8 . \times 10^{-8}$

Chapter $V$

$\operatorname{Ar}_{2} F^{\star}$ Quenching

$$
\begin{array}{lll}
\operatorname{Ar}_{2} \mathrm{~F}^{\star}+\mathrm{F}_{2} \rightarrow-2 \mathrm{Ar}+3 \mathrm{~F} & 1.8 \times 10^{-10} \mathrm{~A} & \text { Chapter } V \\
\operatorname{Ar}_{2} \mathrm{~F}^{\star}+\mathrm{Ar} \rightarrow 3 \mathrm{Ar}+\mathrm{F} & <10^{-14} \mathrm{~A} & \text { Chapter } V \\
\operatorname{Ar}_{2} \mathrm{~F}^{\star}+\mathrm{e}^{-}-2 \mathrm{Ar}+\mathrm{F}+\mathrm{e}^{-} & 2 . \times 10^{-8} & \text { Chapter } V \\
\text { Note } A=\left(\mathrm{T}_{\mathrm{g}} / 300^{\circ} \mathrm{K}\right)^{.5} & &
\end{array}
$$
(a) $M=H e, R=.4 ; M=A r, R=1 ; M=K r, R=.8$
(b) $M=H e, R=1$; $M=A r, R=1$.
(c) $M=H e, R=.4 ; M=A r, R=1.0 ; M=K r, R=1$. 
Table II (continued)

\section{Reaction Kinetics}

Reaction

$k_{R}\left(\mathrm{~cm}^{3} / \mathrm{s}\right.$ or $\left.\mathrm{cm}^{6} / \mathrm{s}\right)$

Source

ArKrF* Quenching

\begin{tabular}{ll} 
ArKrF*+F $2-\mathrm{Ar}+\mathrm{Kr}+3 \mathrm{~F}$ & $1 . \times 10^{-10} \mathrm{~A}$ \\
ArkrF*+Kr $-\mathrm{Kr}_{2} \mathrm{~F}^{\star}+\mathrm{Ar}$ & $3 . \times 10^{-10} \mathrm{~A}$ \\
Radiative Decay & \\
\hline
\end{tabular}

rransition

Lifetime (iis)

Source

$\mathrm{KrF}^{\star} \rightarrow \mathrm{Kr}+\mathrm{F}$

6.8

$(99,100,102)$

$\mathrm{ArF}^{*} \rightarrow \mathrm{Ar}+\mathrm{F}$

4.2

(102)

$A r K r F^{\star}-A r+K r+F$

20.

$\mathrm{Kr}_{2} \mathrm{~F}^{\star}-2 \mathrm{Kr}+\mathrm{F}$

220 .

Chapter V

$\mathrm{Ar}_{2} \mathrm{~F}^{\star}-2 \mathrm{Ar}+\mathrm{F}$

200.

Chapter V

$\mathrm{Kr}_{2} *\left({ }^{3} \Sigma\right)-2 \mathrm{Kr}$

670.

$\mathrm{Kr}_{2}{ }^{\star}\left({ }^{\top} \Sigma\right)-2 \mathrm{Kr}$

5.9

$\mathrm{Ar}_{2} *\left({ }^{3} \Sigma\right)-2 \mathrm{Ar}$

3,100 .

$\mathrm{Kr}_{2}{ }^{*}\left({ }^{\mathrm{I}} \Sigma\right)-2 \mathrm{Ar}$

4.2

$(103,104)$

$F^{\star}(3 p)-F^{\star}(3 s)$

24.

He $2^{7} \mathrm{P}-\mathrm{He}$

.55

He $2^{7} \mathrm{P}-\mathrm{He} 2^{1} \mathrm{~S}$

500.

He $2^{3} \mathrm{p}-\mathrm{He} 2^{3} \mathrm{~s}$

100.

$(103,104)$

Note $A=\left(T_{g} / 300^{\circ} \mathrm{K}\right) \cdot 5$

(a) Rate estimated from the corresponding rate for the other rare gas trimers 
Table III

Photoacsorption Cross Sections at $248 \mathrm{~nm}$.

\begin{tabular}{|c|c|c|c|}
\hline Absorber & $110^{-}$ & $\left.{ }^{18} \mathrm{~cm}^{2}\right)$ & Source \\
\hline$F^{-}$ & 5.5 & & $(110)$ \\
\hline$F_{2}$ & .013 & & $(57,11)$ \\
\hline $\mathrm{F}_{2}$ * & .013 & $(a)$ & $(57,111)$ \\
\hline He $2^{3} \mathrm{~s}$ & 4.8 & & (112) \\
\hline $\mathrm{Kr} \mathrm{5s}$ & .013 & & $(112,113)$ \\
\hline $\mathrm{kr} 5 \mathrm{p}$ & 4.3 & & $(113)$ \\
\hline $\operatorname{Ar} 4 \mathrm{~s}$ & .015 & & $(112,113)$ \\
\hline Ar $4 p$ & 6.0 & & $(113)$ \\
\hline $\mathrm{Kr}_{2}^{+}$ & 1.8 & (b) & $(114)$ \\
\hline $\mathrm{Kr}_{2}{ }^{*}$ & 1.8 & (c) & (114) \\
\hline $\mathrm{Kr}_{2} \mathrm{~F}^{\star}$ & 1.8 & (c) & $(114)$ \\
\hline $\mathrm{Ar}_{2}^{+}$ & 10 & (b) & (114) \\
\hline $\operatorname{Ar}_{2}{ }^{*}$ & 10. & (c) & $(114)$ \\
\hline $\mathrm{Ar}_{2} \mathrm{~F}^{*}$ & 10. & (c) & (114) \\
\hline
\end{tabular}

(a) Cross section is assumed the same as for $F_{2}$

(b) Cross section is a function of the gas temperature. value quoted is for $\mathrm{T}_{\mathrm{g}}=300^{\circ} \mathrm{K}$.

(c) Cross section is assumed equal to that for the rare gas dimer ion transition. 
Table IV

Steady State Results for a Uniformly

Pumped Laser (see Eq. 11)

$\frac{\text { Input Flux }}{\text { Saturation Flux }} \quad \frac{\text { Output Flux }}{\text { Saturation Flux }}$

$\mathrm{g}_{0}=.1$

$\begin{array}{cc}.1 & .10947 \\ 1 & 1.0506 \\ 10 & 10.091\end{array}$

$g_{0}=1$.

.1

.23702

1

1.5571

10

10.913

$g_{0}=10$.

.1

$6.00,48$

1

8.8227

10

19.340

$g_{0}=100$

$\begin{array}{rr}.1 & 93.262 \\ 1 & 96.431 \\ 10 & 107.62\end{array}$




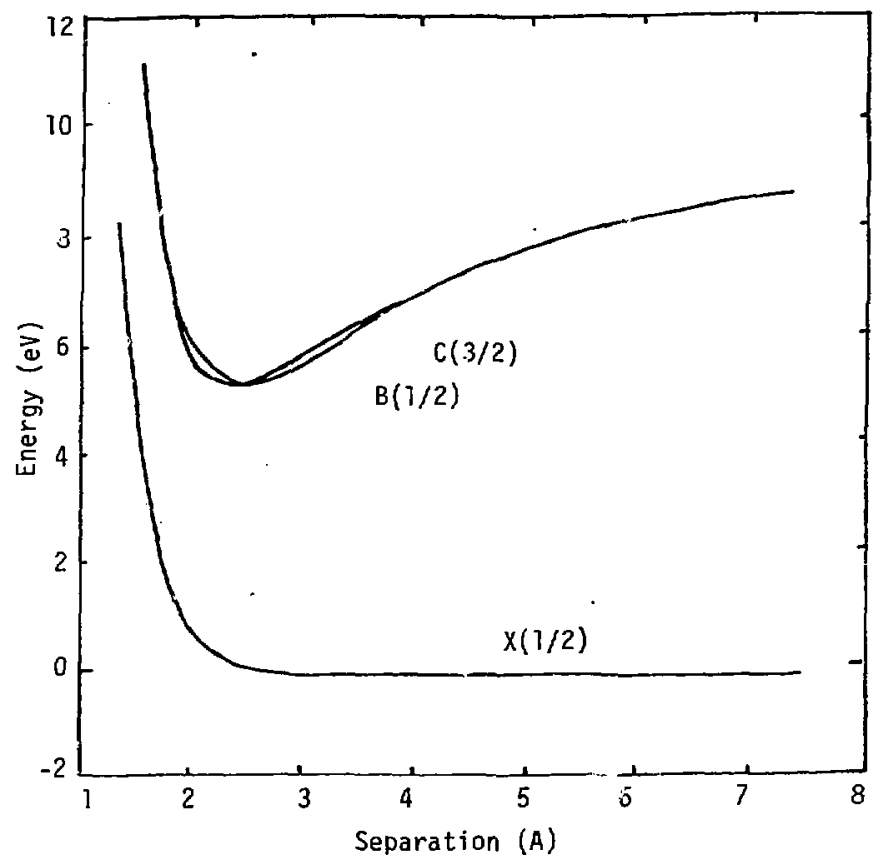

Fig. 1. Nuclear potentici curves for $\mathrm{KrF}$, taken from the work of Hay and Dunning. (107) The KrF laser operates on the $B(1 / 2)-x(1 / 2)$ transition. The $C(3 / 2)$ population serves primarily as a reservoir for the upper Tevel excitation. 


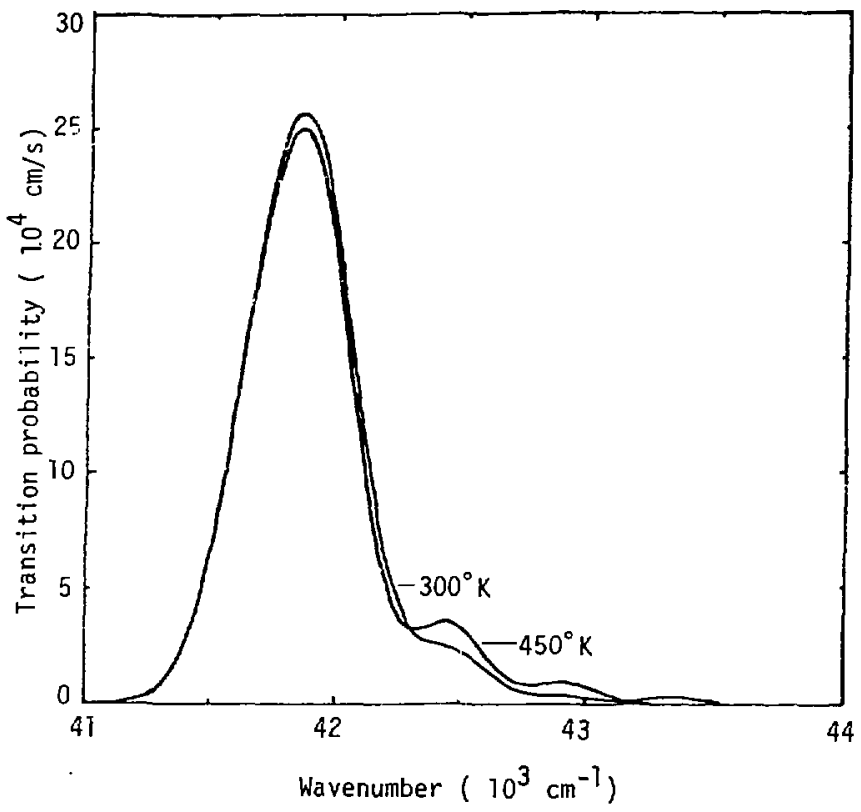

Fig. 2. Calculated spontaneous transition probability vs wavenumber for the $\mathrm{KrF} \mathrm{B} \mathrm{-} \mathrm{X} \mathrm{laser} \mathrm{transition} \mathrm{at}$ 300 and $450^{\circ} \mathrm{K}$. 


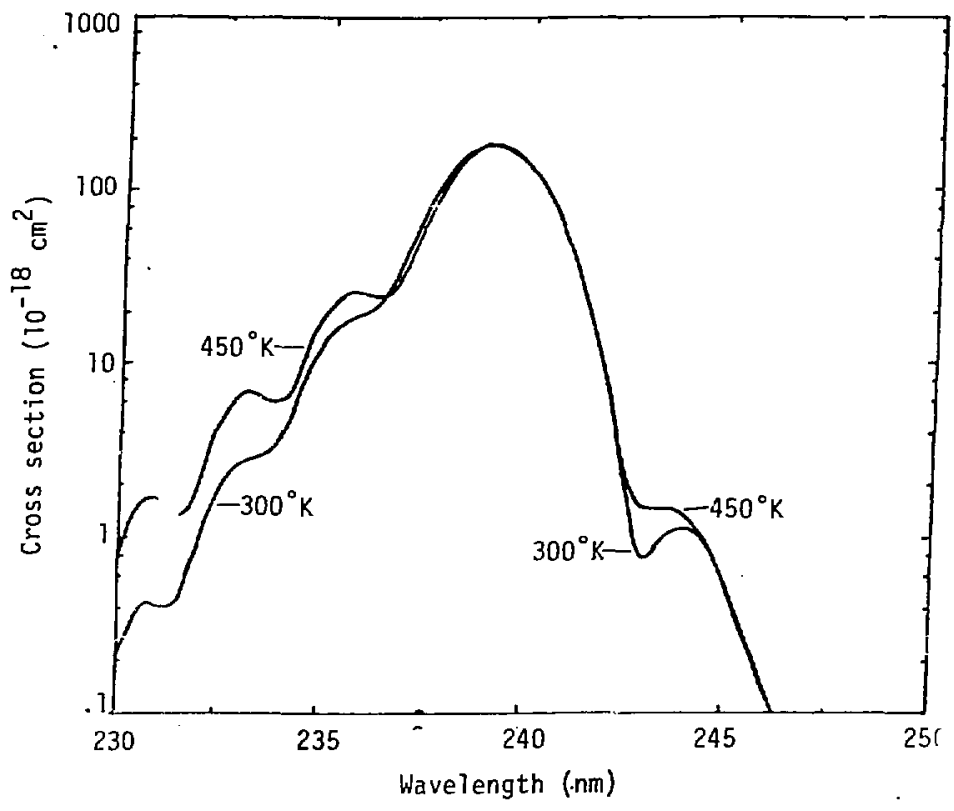

Fig. 3. Calculated stimulated emission cross section vs wavelength for the $\mathrm{KrF} \mathrm{B} \mathrm{-} \mathrm{X} \mathrm{laser} \mathrm{transition} \mathrm{at}$ 300 and $450^{\circ} \mathrm{K}$. 


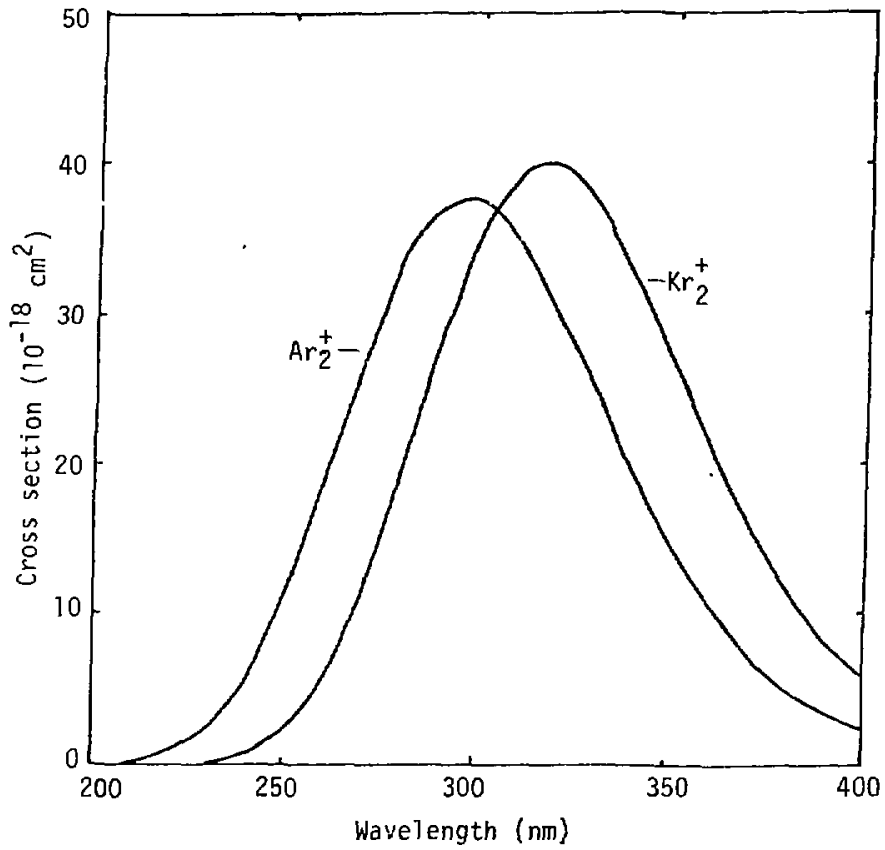

Fig. 4. Calculated $\mathrm{Ar}_{2}^{+}$and $\mathrm{Kr}_{2}^{+}$photoabsorption cross sections vs wavelength at $300^{\circ} \mathrm{K}$. 


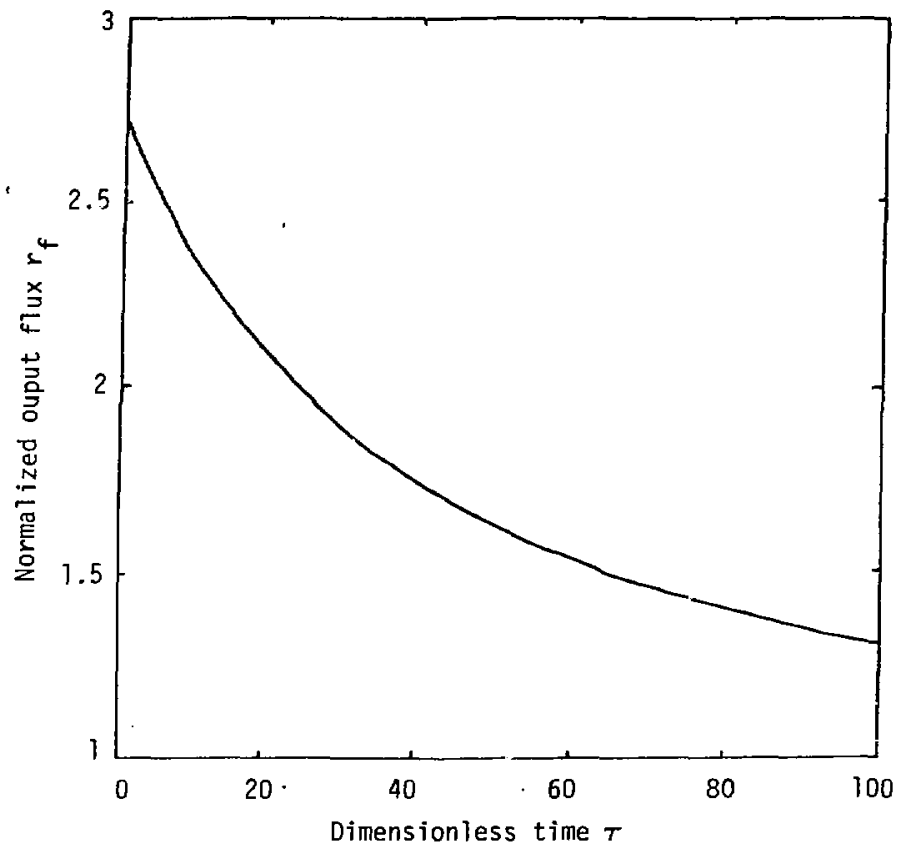

Fig. 5. Result of the second test of the photon transport method described in the text. The difference between the analytic solution for the output flux and that predicted by the model is ress than the width of the plotted curve. 


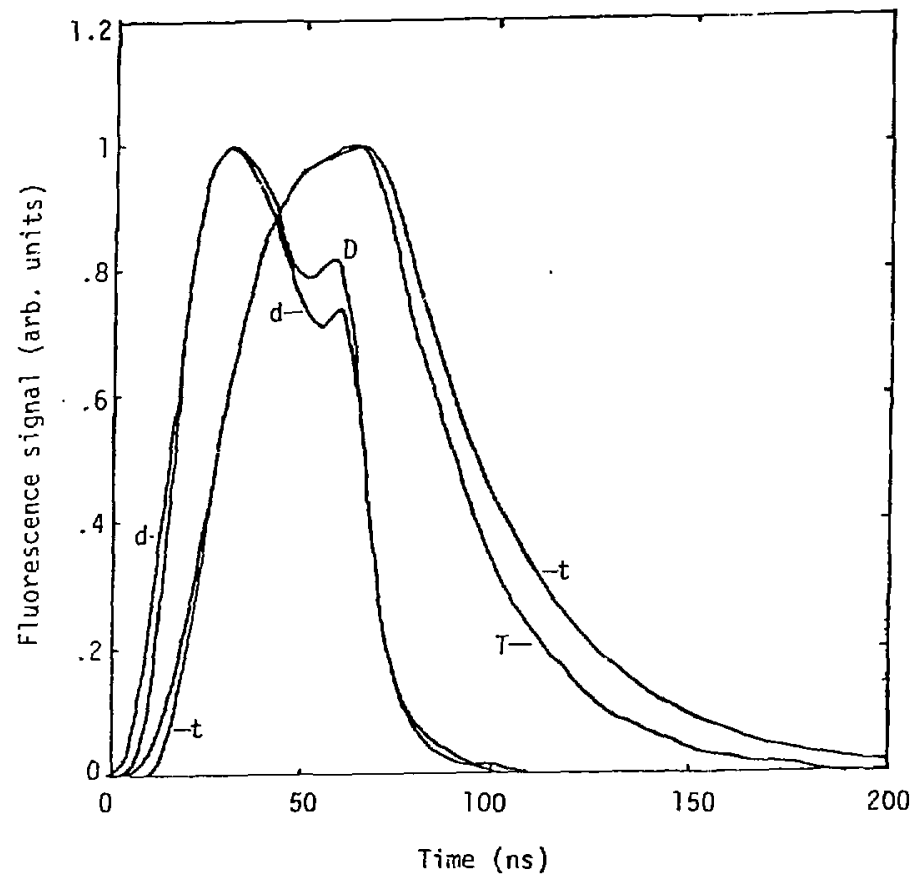

Fig. 6. Comparison of the calculated and experimental dimer and trimer fluorescence signals from a 1500 torr $\mathrm{Ar}, 100$ torr $\mathrm{Kr}, 5$ torr $F_{2}$ gas mix at $33 \mathrm{~A} / \mathrm{cm}^{2}$ : (d) $\mathrm{KrF}$ (calc), (D) KrF (exp); (t) $\mathrm{Kr}_{2} F(\mathrm{calc}),(\mathrm{T}) \mathrm{Kr}_{2} \mathrm{~F}$ (exp). All signals are normalized to the same peak value. 


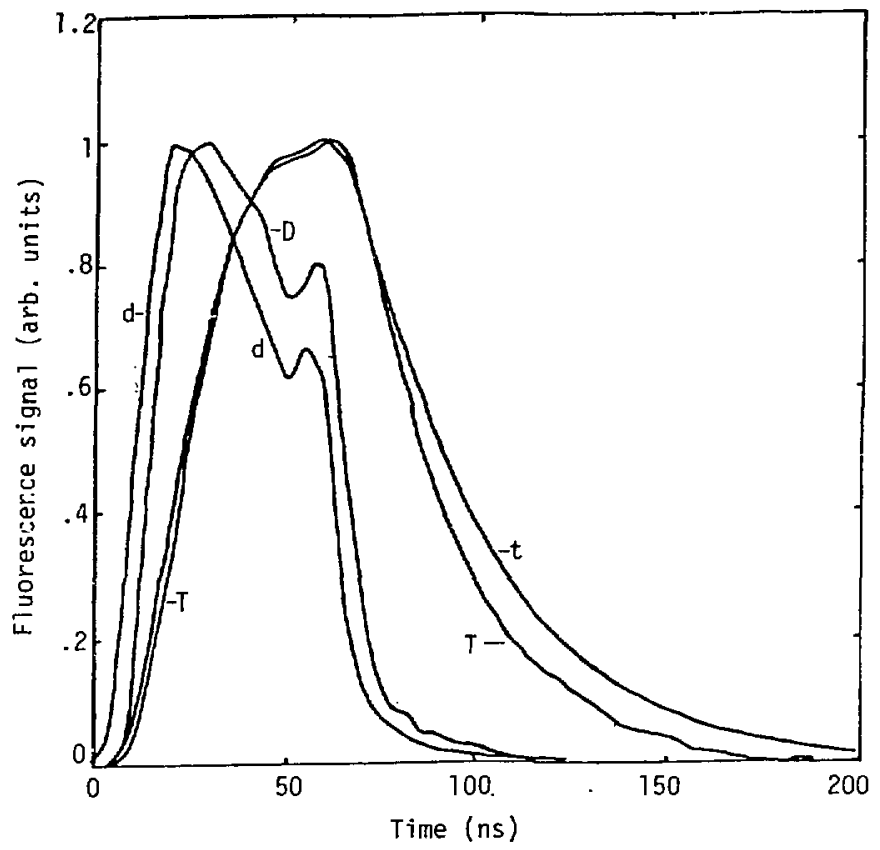

Fig. 7. Comparison of the calculated and experimental dimer and trimer fluorescence signals from a 11,600 torr He, 100 torr $\mathrm{Kr}, 5$ torr $\mathrm{F}_{2}$ gas mix at $33 \mathrm{~A} / \mathrm{cm}^{2}$ : (d) $\mathrm{KiF}$ (calc), (D) $\mathrm{KrF}$ (exp); (t) $k r_{2} F(c a l c)$, (T) $k r_{2} F$ (exp). All signals are normalized to the same peak value. 


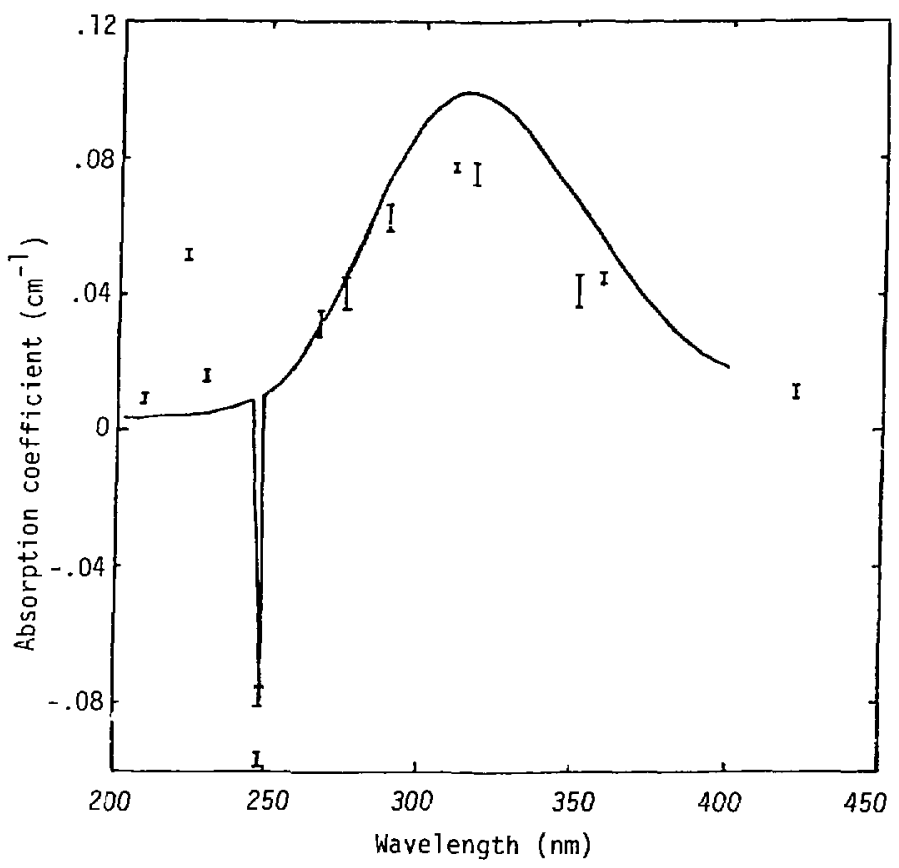

Fig. 8. Spectral dependence of the absorption in a 1500 torr $\mathrm{Ar}, 100$ torr $\mathrm{Kr}, 5$ torr $\mathrm{F}_{2}$ gas mixture at the end of a $140 \mathrm{~A} / \mathrm{cm}^{2}$ current pulse. The solid line is the result of the calculation described in the text. The error bars are the experimental results of Powel1. 116 


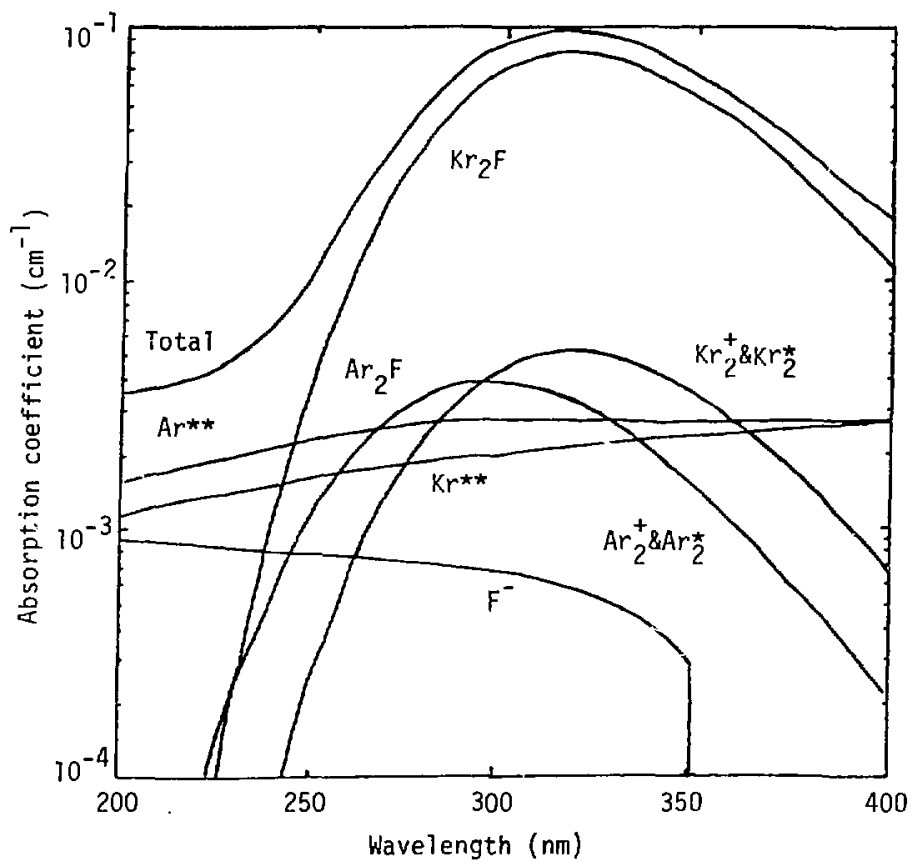

Fig. 9. Breakdown of the calculated absorption cross section shown in Figure 8 , by absorbing species. 
References - Chapter IV

1. C. W. Werner et. al., IEEE. Jrnl Quant. Elect. 13, 769 (1979)

2. W. L. Morgan ano A.Szoke, Phys. Rev. A23, 1256 (1981)

3. C. J. Elliot and A. E. Green, Jrn1. Appl. Phys. 47, 2946 (i976)

4. W. L. Morgan, private communication (1981)

5. J. C. Light et. al., Kinetic Processes in Gases and Plasmas, A.

R. Hochstim, ed. (Academic Press, New York, 1969) p310

¿. I. P. Shkarofsky et. al., The Particle Kinetics of Plasmas

(Addison Wesley, Reading, 1966) Sec. 3-5

7. L. Spitzer, Ir., Physics uf Fully Ionized Gases (John wiley and Sons, New York, 1962) pi35

8. D. L.Bunker, Theory of Elementary Reaction Rates (Pergammon, New York, 1966) pl4

9. G. Hammes, Principles of Chemical Kinetics (Academic Press, New York, 1978) p4 1

10. M. R. Flannery and T. P. Yang, Appl. Phys. Lett. 32, 32; (1978)

11. E. W. McDaniel, Collision Phenomena in Ionized Gases (John Wiley and Soris, New York, 1964) p570

12. E. W. McDaniel and E. A. Mason, The Mobility and Diffusion of Ions in Gases (John Wiley and Sons, New York, 1973) p344, pi21, p 134

13. H. W. Ellis et. al., Atomic and Nuclear Data Tables 17, 177 (1976)

14. I. Dotan et. al., Jrnl. Chem. Phys. 66, 2232 (1977)

15. I. Dotan and D. L. Albritton, Jrnl. Chem. Phys. 66, 5238 (1977) 
16. J. R. Murray and H. T. Powel1, App1. Phys. Lett. 29, 252 (1976)

17. A. E. S. Green and C. A. Barth, Jrnl. Geophys. Res. 70, 1083 (1965)

18. D. Smith et. al., Jrn1. Phys. B5,2134 (1972)

19. 3. C. Tracy and H. J. Oskam, Jrnt. Chem. Phys. 65, 3387 (1976)

20. J. C. Tracy and H. J. Oskam, Jrn7. Chem. Phys. 65, 1666 (1976)

21. C. W. Werner, Ph. D. Thesis, Massachusetts Institute of Technology (1975)

22. R. S. F. Chang and D. W. Setzer, Jrnl. Chem. Phys. 69, 3885 (1978)

23. D. K. Bohme et. al., Jrnl. Chem. Phys. 52, 5094 (1970)

24. L. G. Piper et. a1., Jrnl. Chem. Phys. $\underline{59}, 3323$ (1973)

25. L. G. Piper et. al., Jrnt. Chem. Phys. 63, 5018 (1975)

26. H. H. Nakano et. al., S. R. I. Report MP 76-99 (1976)

27. T. Oka et. al., Irnt. Chem. Phys. 70, 3384 (1979)

28. D. Smith and P.R. Cromey, Jrnl. Phys. B], 638 (1968)

29. E. W. McDaniel et. al., Ion Molecule Reactions (Wiley Interscience, New York, 1970) p338

30. 8. M. Smirnov, Sov. Phys - JETP 24, 1180 (1967)

31. D. C. Lorents, Physica 82C, 19 (1976)

32. E. Ellis and N. D. Twiddy, JrnT. Phys. 82, 1366 (1969)

33. G. S. Hurt et. al., Jrn1. Chem. Phys. 61, 3680 (1974)

34. M. V. McCusker, Excimer Lasers, C. K. Rhodes, ed. (Springer Verlag, New York, 1979) p47

35. E. Zamir et. a1., App1. Phys. Lett. 27, 56 (1975)

36. E. W. McDaniel et. al., Ion Molecule Reactions (Wiley Interscience, New York, 1970) p338 
37. W. Lindinger et. al., Jrnt. Chem. Phys. 61, 2890 (1974)

38. H. Hotop et. al., Z. Phys 229, I (1969)

39. H. L. Kramer et. al., Jrnl. Chem. Phys. 56, 4166 (1972)

40. F. W. Lee and C. B. Collins, Jrnl. Chem. Phys. 65, 5189 (1977)

41. M. H. Nayfeh et. al., Phys. Rev. Al4, 1739 (1976)

42. F. W. Lee and C. B. Collins, Jrnl. Chem. Phys. 67, 2798 (1977)

43. F. W. Lee et. al., Jrnl. Chem. Phys. 65, 1605 (1976)

44. E. C. Beatty and P. L. Patterson, Phys. Rev. 137, A346 (1965)

45. P. L. Patterson, Jrnl. Chem. Phys. 48,3625 (1968)

46. R. Deloche et. al., Phys. Rev. A13, 1140 (1976)

47. G. Meyers and A. J. Cunningham, Jrn . Chem. Phys. 67, 247 (1977)

48. J. E. Lawler et. al., Phys. Rev. Lett. $\underline{39}, 543$ (1977)

49. D. M. Bartell et. al., Phy: . Rev. A8, 1068 (1973)

50. M. G. Payne et. a]., Phys. Rev. Lett. $\underline{35}$, i154 (1975)

51. M. Gand et. al., Appl. Phys. Lett. $\underline{35}, 50$ (1979)

52. L.J. Kieffer, A Compilation of Electron Collisional Cross Section Data for Modelling Gas Discharge Lasers, JILA Information Center Rept. 13, U. Colorado (1973)

53. J. R. Gibson and K. Y. Dolder, Jrnl. Phys. B2, 1180 (1969)

54. H. L. Chen et. al., Appl. Phys. Lett. 30,99 (1977)

55. D. W. Trainor and J. H. Jacob, Appl. Phys. Lett. $\underline{35}, 920$ (1979)

56. R. J. Hall, Jrn1. Chem. Phys. 68, 1803 (1978)

57. D. C. Cartwright and P. J. Hay, Jrni. Chem. Phys. 70, 3191 (1979)

58. J. N. Bardsley and M. A. Biondi, Advances in Atomic and Molecular Physics, Vol 6. (Academic Press, New York, 1970) plff

59. T. F. O'Malley, Phys. Rev. 185, 101 (1969) 
60. H. J. Oskam and V. R. Mittelstadt, Phys. Rev. 132, 1443 (1963)

61. Y. J. Shiu and M. A. Biondi, Phys. Rev. Al6, 1817 (1977)

62. C. J. Powell, Rev. Mad. Phys. 48, 83 (1976)

63. O. Rapp and P. Englander-Golden, Jrnl. Chem. Phys. 43, 1464 (1965)

64. Laser Fusion Program Annual Report - 1978 Vol. 3, UCRL 50021-78, Lawrence Livermore Lahoratory (1978) p8-6

65. D. Ton-That and M. R. Flannery, Phys. Rev. A15, 517 (1977)

65. T. A. Johnson, AFWL-TR-77-15, A.ir Force Weapons Laboratory (1977), p100

67. R. E. Fox, Jrn1. Chem. Phys. $\underline{33}, 200$ (1960)

68. R. E. Fox, Jrnt. Chem. Phys. 35, 1379 (1961)

69. A. J. Dixon et. al., Jrnl. Phys. B9, 2617 (1976)

70. D. R. Long and R. Geballe, Phys. Rev. Al, 260 (1970)

71. R. A. Hyde and M. G. Roberts, UCID 1777'. Lawrence Livermore Laboratory (1978)

72. M. Rockni et. al., IEEE Jrnl. Quant. Elect. 14, 464 (1978)

73. P. S. Ganas and A. E. S. Green, Phys. Rev. A4, 182 (1971)

74. V. Raible and H. Koschmeider, Jrnl. Phys. B7, L99 (1974)

75. E. Eggarter, Jrn1. Chem. Phys. 62, 833 (1975)

76. L. R. Petterson and J. E. Allen, Jrni. Chem. Phys. 56, 6068 (1972)

77. J. W. McConkey and F. G. Donaldson, Can. Jrnl. Phys. 51, 914 (1973)

78. S. Trajmar, Phys. Rev. A8, 191 (1973)

79. W. Borst, Phys. Rev. A9, 1195 (1974)

80. K. Hall et. al., Jrnl. Phys. BE, 66 (1972)

81. M. Crooks et. al., Phys. Rev. Lett. 29,327 (1972) 
82. W. C. Fon et. a]., Jrn1. Phys. B12, 1861 (?979)

83. R. Bell et. a7., Jrn]. Phys. B2, 26 ( '39)

84. J. Vriens et. al., Phys. Rev. 165, 7 !1972)

85. T. Rice et. al., Pnys. Rev. A5, 762 (1972)

86. J. D. Jobe and R. M. St, John, Phys. Rev. 164, 117 (1967)

87. F. G. Donaldson et. al., Jrn1. Phys. B5, 11y2 (1972)

88. M. A. Dillon and E. N. Lasettre, Jrnl. Chem. Phys. 62, 2373 (1975)

89. P. G. Burke et. al., Phys. Rev. 183, 245 (1969)

90. M. R. Flannery and T. P. Yang, Appl. Phys. Lett. 33 , 574 (1978)

91. M. R. Flannery and T. P. Yang, Appl. Phys. Lett. 33, 356 (1978)

92. C. H. Chen et. a]., Jrn]. Phys. Bi i, 2189 (1978;

93. $\therefore$ A. Brau, Excimer Lasers, C. K. Rhodes, ed. iSpringer Verlag, New York, 1979) p87

94." J. E. Velazco et. al., Jrn1. Chem. Phys. 65, 3468 (1976)

95. G. P. Quigley and W. M. Hughes, Appl. Phys. Lett. $\underline{32}, 649$ (1978)

96. C. H. Chen et. a]., Jrn]. Chem. Phys. 69, 1626 (1978)

97. M. Rokni et. a]., Appl. Phys. Lett. 31, 79 (1977)

98. J. Mangano, private communication (1981)

99. J. G. Eden et. al., Appl. Phys. Lett. $\underline{32}, 733$ (i978)

100. G. P. Quigley and W. M. Hughew, App 1. Phys. Lett. 32, 627 (1978)

101. M. Rokni et. al., Phys. Rev. Al6, 2216 (1977)

102. T. H. Dunning and P. J. Hay, Jrn]. Chem. Phys. 69, 134 (1978)

103. ․ W. Keto et. a]. ,Chem. Phys. Lett. 42, 125 (1976)

104. J. W. Keto et. a]., Phys. Rev. Lett. 33, 1365 (1974)

105. W. L. Wiese et. al., Atomic Iransition Probabilities, NSRDS-NB44, National Bureau of Standards (1966) 
106. M. Born and J. Oppenheimer, Annal. Phys. 84, 457 (1927)

107. P. J. Hay and T. H. Dunning, Jr., Jrni. Chem. Phys. 66, 1306 (1977)

108. F. H. Mies, Molec. Phys. 26, 1233 (1973)

109. Laser Fusion Program Annual Report - 1976, UCRL 50021-76, Lawrence Livermore Laboratory (1976) p6-65

110. A. Mandl, Phys. Rev. A3, 251 (1971)

111. R. K. Steunenberg and R. C. Vogel, Jnrl. Amer. Chem. Soc. 78, 901 (1956)

112. K. J. McCann and M. R. Flannery, Appl. Phys. Lett. 31, 599 (1977)

113. H. A. Hyman, Appl. Phys. Lett 31, 14 (1977)

114. H. H. Michels et. al., Jrnl. Chem. Phys. 69, 5151 (1978)

115. C. W. Gear, Numerical Initial Value Problems in Ordinary Differential Equations (Prentice Hall, Englewood Cliffs, 1971)

116. H. T. Powell, private communication (1981)

117. Laser Fusion Program Annual Report - 1980, UCRL 50021-80, Lawrence Livermore National Laboratory (1980) p8-35 


\section{Chapter V}

The Formation and Quenching of $\mathrm{Ar}_{2} \mathrm{~F}^{\star}$ and $\mathrm{Kr}_{2} \mathrm{~F}^{\star}$

\section{A. Introduction}

The rare gas halide (RGH) trimers $\mathrm{Ar}_{2} \mathrm{~F}^{\star}$ and $\mathrm{Kr}_{2} \mathrm{~F}^{\star}$ are important species in krypton fluoride laser media. Their creation from the RGH dimers represents either an interception of the gas excitation en route to the laser upper state (e.g. in the formation of $\mathrm{Ar}_{2} \mathrm{~F}^{*}$, rather than $\mathrm{KrF}^{*}$, from $\mathrm{ArF}^{*}$ ) or a loss mechanism from the laser state via the formation of $\mathrm{Kr}_{2} \mathrm{~F}^{*}$ from $\mathrm{KrF}^{*}$. In addition, the large concentrations of $\mathrm{RGH}$ trimers formed in the gas may contribute significantly to its optical absorption coefficient at the laser wavelength, as demonstrated in the preceeding chapter.

The formation and quenching of the RGH trimers has been studied at Lawrence Livermore National Laboratory by this author along with Dr. H. T. Powell. A paper describing our work on the trimer kinetics of e-beam excited mixtures of either Ar or $\mathrm{Kr}$ combined with either $\mathrm{F}_{2}$, $\mathrm{NF}_{3}$, or $\mathrm{SF}_{6}$ is presented in the Appendix to this chapter. Buffered gas mixtures containing $\mathrm{Kr}, \mathrm{F}_{2}$ and either $\mathrm{He}$, Ne, or $\mathrm{Ar}$ have also been examined, and are discussed in Section B of this chapter. 
The experimental apparaius designed by Dr. Fowell to both excite the gas mixtures and observe the resultant fluorescence is desc, ibed in Section II of the Appendix. A unique feature of this setup is the ease with which the gas mixture, total gas pressure, and electron beam current density could all be varied over a wide range, while the electron energy and electron beam pulse shape remained roughly constant. Variations in average current density of 2 to 500 Amp $/ \mathrm{cm}^{2}$, and in total pressure of 200 to 20,000 Torr were attainable.

For the purpose of ueiermining the trimer formation and destruction kinetics, measurement of both the RGH dime - and trimer time-dependent fluorescence decays was required. The experimental results obcained by Dr. Powell for binary gas mixtures are described in Section III of the Appendix. Similar data obtained by this author for buffered gas mixtures is discussed in Section B of this chapter.

In either binary mixtures of a rare gas with a fluorine donor, or in buffered mixtures where the buffer gas does not form a bound excited state with fluorine (i.e. with either helium or neon as the buffer), both theoretical arguments ${ }^{\prime}$ and experimental measurements 2,3 indicate that the RGH trimers are formed solely by three-body reactions with the corresponding dimer molecule (e.g. $\mathrm{Kr}_{2} \mathrm{~F}^{*}$ from $\left.\mathrm{KrF}^{*}\right)$. In this case, the trimer kinetics simplifies greatly in that the time dependence of the trimer formation rate is determined solely by the relevant dimer concentration. It is this simplification that allows us to develop a simple model which ignores 
the complicated processes involved in the transfer of energy from the e-beam to the RGH dimer molecule, and permits us to unambiguously determine the RGH trimer formation and decay rate constants.

The analysis developed by this author to determine the RGH trimer kinetic rate coefficients from the measurements of the dimer and trimer fluorescence signals is presented in Section IV of the Appendix. The results of this analysis when applied to the binary gas mixture data are discussed in Section $V$. Section $B$ of this chapter contains the results obtained for the helium- and neon-buffered gas mixtures, and in addition dicusses the results obtained for argon-buffered mixtures, where the assumption that $\mathrm{KrF}$ is the only precursor to the $\mathrm{Kr}_{2} \mathrm{~F}^{\star}$ trimer does not apply

B. Buffered $\underline{\mathrm{Kr}-\mathrm{F}} 2$ Gas Mixtures

The expe"imental technique described in Section II of the Appendix was used to measure the $\mathrm{KrF}^{\star}$ and $\mathrm{Kr}_{2} \mathrm{~F}^{\star}$ flucrescence signais from $20 / 1 \mathrm{Kr} / \mathrm{F}_{2}$ gas mixtures buffered with either helium, neon or argon excited at low current density $\left(\approx 33 \mathrm{Amp} / \mathrm{cm}^{2}\right)$. To simplify the comparison of the performance using different buffers, the partial pressure ratios (He:Kr:F $F_{2}=1,897: 20: 1$; Ne:Kr:F $=422: 20: 1$; and $A r: K r: F 2=246: 20: 1)$ were chosen to yield the same energy deposition per unit volume from the $600 \mathrm{keV}$ e-beam pulse as a "standard" $150 / 1 \mathrm{Kr} / \mathrm{F}_{2}$ mixture at identical $F_{2}$ partial presssures, as calculated using the stopping powers of Berger and Selzer. ${ }^{4}$ 
Both the $\mathrm{KrF}^{*}$ and $\mathrm{Kr}_{2} \mathrm{~F}^{*}$ fluorescences from all of the buffered mixtures were qualitatively similar to those from the $\mathrm{kr}-\mathrm{F}_{2}$ binary mixtures described in Section III of the Appendix. The peak dimer and trimer signals as a function of the average energy deposition rate for each three-component gas mix, along with those from the "standard" $\mathrm{Kr}-\mathrm{F}_{2}$ mixture are presented in Figures 1 and 2 respectively. The energy deposition was assumed to be twice that indicated by the stopping powers of Berger and Selzer ${ }^{4}$ for a single pass, as suggested by detailed numerical calculations using the three-dimensional deposition code SANDYL. 5

The magnitude of both of the fluorescence signals changes with the buffer yas used for a number of reasons. First, as the average $z$ of the mixure is reduced the energy of the average initial excitation produced by the e-beam increases, as indicated by the solutions of the Fowler equation discussed in Chapter 11. This implies that a fewer excitations are produced in the lower- $Z$ gas mixtures at a given energy deposition rate, which would then yield a smaller $\mathrm{KrF}^{\star}$ production rate at a given yield of RGH dimers produced per initial excitation. Secondly, as the buffer gas is changed the permitted energy pathways for transfer of energy into the $\mathrm{KrF}^{\star}$ excimer themselves vary. For example, with helium as the buffer the energy in the excited states of the buffer atoms may be rapidly transfered directly to the krypton atoms via Penning ionization, which is not possible when either neon or argon is used. Finally, the kinetics of the RGH dimer-trimer system itself changes, due to the increased 
pressures required with the lower $-Z$ gases, changes in the magnitudes of the kinetic rate constants, and differences in the available kinetic pathways (e.g. the formation of ArKrF* from KrF*, which has no analog for either helium or neon).

The low current density analys is developed in Section IV of the Appendix for binary gas mixtures was applied to the fluorescence signals from the buffered mixes to determine the changes in the $\mathrm{KrF}^{*}-\mathrm{Kr}_{2} \mathrm{~F}^{\star}$ kinetics witn the various buffer gases. The best fit values of the trimer decay rate $\tau^{-1}$ determined from this analysis are plotted vs the total gas pressure in Figures 3, 4, and 5 for the helium, neon, and argon mixtures, respectively. The solid lines in each of the figures are the results of a least squares fit of the decay rate to a form linear in the gas pressure; in all cases the intercepts are consistent with the 225 nsec. radiative lifetime for $\mathrm{Kr}_{2} \mathrm{~F}^{\star}$ obtained in the analysis of the binary gas mixture data in the A.ppendix.

Assuming that the pressure dependence of $\tau^{-1}$ is given by

$$
\tau^{-1}=T_{\text {rad }}^{-1}+k_{K r} n_{K r}+k_{\text {buf }} n_{\text {buf }}+k_{F_{2}}{ }^{n_{F}}
$$

where the " $n$ ' $s$ " represent the concentrations of the various gas species, and using the values of $k_{K r}$ and $k_{F_{2}}$ given in Table 2 of the Appendix, the linear fits shown in Figures 3-5 yielded values for the rate constants for the quenching of $\mathrm{Kr}_{2} \mathrm{~F}^{\star}$ by the buffer 
gases. In all cases the pressure dependent quenching was dominated by its flourine contribution $(>80 \%)$, allowing us to obtain only rough estimates for the values of $k_{b u f}$. For helium we calculate $k_{\mathrm{He}}=(2 \pm 1) \times 10^{-14} \mathrm{~cm}^{3} \mathrm{~s}^{-1}$ while for neon and argon we could only place an upper bound on $k_{\mathrm{Ne}}$ and $k_{\mathrm{Ar}}$ of $10^{-13} \mathrm{~cm}^{3} \mathrm{~s}^{-1}$.

The low current density analys is developed in the Appendix is strictly applicable only when the sole kinetic pathway into $\mathrm{Kr}_{2} \mathrm{~F}^{*}$ is directly from the $\mathrm{KrF}^{*}$ dimer. One may then question $i$ is use in the anilys is of the data with argon as the buffer gas, as the additional triner formation channel

$$
\begin{aligned}
& \mathrm{KrF} *+2 \mathrm{Ar}-\mathrm{ArKrF} *+\mathrm{Ar} \\
& \mathrm{ArKrF} *+\mathrm{Kr}-\mathrm{Kr}_{2} \mathrm{~F}^{*}+\mathrm{Ar}
\end{aligned}
$$

(with the reaction rate constants $k_{2 A r}$ and $k_{\text {disp }}$, respectively) is available in this case. However, at the krypton partial pressures of interest here (>25 torr), the value of $k_{\text {disp }}=3 \times 10^{-10} \mathrm{~cm}^{3} \mathrm{~s}^{-1}$ quoted in Chapter 5 indicates that the lifetime of ArKrF* for decay via displacement is less than $4 \mathrm{nsec}$., a value considerably less than both the characteristic time required for a significant change in the KrF* dimer concentration and the ArKrF* radiative lifetime. We would therfore expect the ArKrF* concentration to be in approximate equilibrium with that of the $\mathrm{KrF}^{*}$ dimer, and to decay with nearly unit efficiency into $\mathrm{Kr}_{2} \mathrm{~F}^{\star}$. The treatment given in Section IV of the 
Appendix then remains valid, provided that we include a term in the trimer formation rate of $\mathrm{Kr}_{2} \mathrm{~F}^{*}$ from $\mathrm{KrF}^{*}$ proportional to the square of the argon concentration.

The values of the trimer formation rati $\boldsymbol{\alpha}$ determined in the analysis of the fluorescence data from all of the buffered gas mixtures, as well as that from the "standard" $\mathrm{Kr}-\mathrm{F}_{2} \mathrm{mix}$, are plotted $v s$ the total gas pressure in Figure 6 . For the binary $k r-F_{2}$ mixture, as well as for the helium and neon buffered cases, the assumption that the trimer is formed via three-body collisions would require

$$
\alpha=A_{2 K r^{n}} K^{2}+A_{\text {buf, }}, r^{n}{ }_{b u f} n_{k r}
$$

with $A_{2 K r}=S_{Y} k_{2 K r} / S_{W}$, where $S_{Y}$ and $S_{W}$ are the trimer and dimer detector sensitivity factors derived in the Appendix, and $k_{2 k r}$ is the rate constant for the formation of $K r_{2} F^{\star}$ in a three-body collision of $\mathrm{KrF}$ with 2 krypton atoms. A $A_{\text {buf' }} \mathrm{Kr}$ and $\mathrm{k}_{\text {buf, } \mathrm{Kr}}$ are defined similarly. Though the data deviates slightly from the expected $p^{2}$ dependence implied by this assumption (possibly for the same reasons as suggested in the interpretation of similar results in the Appendix), we have used it to rotain estimates for the relative values of the three body formation rate constants. First, a least-squares fit of Eq. 3 to the values of for the binary $\mathrm{kr}-\mathrm{F}_{2}$ mixture yielded an estimate for $A_{2 K r}$. Using this value in a fit of 
Eq. 3 to the values of $\boldsymbol{\alpha}$ with helium or neon as the buffer then reslited in an estimate for $A_{b u f,},{ }_{r}$, in which case we have

$$
k_{\text {buf, }, \mathrm{Kr}} / \mathrm{k}_{2 \mathrm{Kr}}=A_{\text {buf, } \mathrm{Kr}} / A_{2 \mathrm{Kr}} \quad \text { (4). }
$$

The values obtained in this way for the three-body iormation rate constants are $k_{\mathrm{He}, \mathrm{Kr}}=(.25-.6) \mathrm{k}_{2 \mathrm{Kr}}$, and $\mathrm{k}_{\mathrm{Ne}, \mathrm{Kr}}=(.5-.7) \mathrm{k}_{2 \mathrm{Kr}}$, where the range in each of these values is given by the deviation of from its expected $p^{2}$ dependence.

For the case where argon is used as the buffer, Eq. 4 for the source term $\boldsymbol{\alpha}$ must be modified by adding a term equal to $A_{2 A R^{n}} A r^{2}$ (where $A_{2 A r}$ is defined in the same manner as $A_{2 K r}$ ), which corresponds to the formation of $\mathrm{Kr}_{2} \mathrm{~F}^{*}$ through the ArKrF* channel. In this case the analys is of the pressure dependence of a yieliss only the result

$$
k_{A r, K r}+k_{2 A r}\left(n_{A r} / n_{K r}\right)=k_{A r, K r}+12.3 k_{2 A r}:(2.1-2.5) k_{2 K r}
$$

which is consistent with the values of $k_{A r, k r} \approx k_{2 k r}$ and $k_{2 A r} \approx .13 k_{2 k r}$ quoted in Chapter IV. 


\section{c. Discussion and Conclusions}

The results obtained in the arlalys is of the binary gas mixtures of either kiypton or argon with the various fluorine donors aris reviewed in the main in Section $V I$ of the Appendix. Regarding the excitation dependent quenching of the RGH trimers at high current densities, though we are forced to conclude that it is caused by electrons, we have found no model which can accurately predict the magnitude of its effect. Such a determination appears to require in the least a method for measuring the electron density in the e-beam excited gas on a nanosecond time scale, which to date has eluded us.

In both the binary and buffered gas mixtures the formation of the RGH trimers appears to proceed through three body collisions with the dime: $;$. Estimates for the three-body formation rate constants for the binary gas mixtures and the helium and neon buffered mixtures have been calculated. In the argon buffered $k r-F_{2}$ gas mix, the importance of the kinetic pathway from $\mathrm{KrF}^{*}$ throuyin $\mathrm{ArKrF}^{\star}$ to $\mathrm{Kr}_{2} \mathrm{~F}^{*}$ has been demonstrated, and the trimer formation rate has been shown to be consistent with the accepted rate constants.

At the lower current densities $\left(<50 \mathrm{~A} / \mathrm{cm}^{2}\right)$, the trimer decay rate is dominated by radiative decay and heavy particle quenching in all the cases considered. Values for the trimer radiative lifetimes and either estimates or upper bounds for the heavy particle quenching rate constants have been calculated. In those cases where the 
quenching of the trimer by the fluorine donor was small, we have found indications of quenching of the trimer by dissociation products of the donor.

In retrospect, it is easy to suggest improvements in the experimental work described above. By studying gas mixtures with a smaller relative concentration of the fluorine donors, it might have been possible to calculate values rather than upper bounds for the rate constants for the quenching of the trimers by the rare gas atoms, though it is possible that problems would arise due to both the resultant decrease in the fluorescence signals and the probable depletion of the fluorine donor. More significant is the fact that, if the fluorescence signals for a gas mixture with a different ratio of the argon concentration to krypton and fluorine concentrations had been measured and analyzed, values of the formation rate constants of $\mathrm{Kr}_{2} \mathrm{~F}^{*}$ and ArKrF* in three body collisions involving $\mathrm{KrF}^{*}$ and an argon atom could have been obtained. For the present, however, such corrections have been precluded by a study of the performance of $\mathrm{krF}$ * laser mixtures as laser mixtures, which is treated in the follawing chapter. 


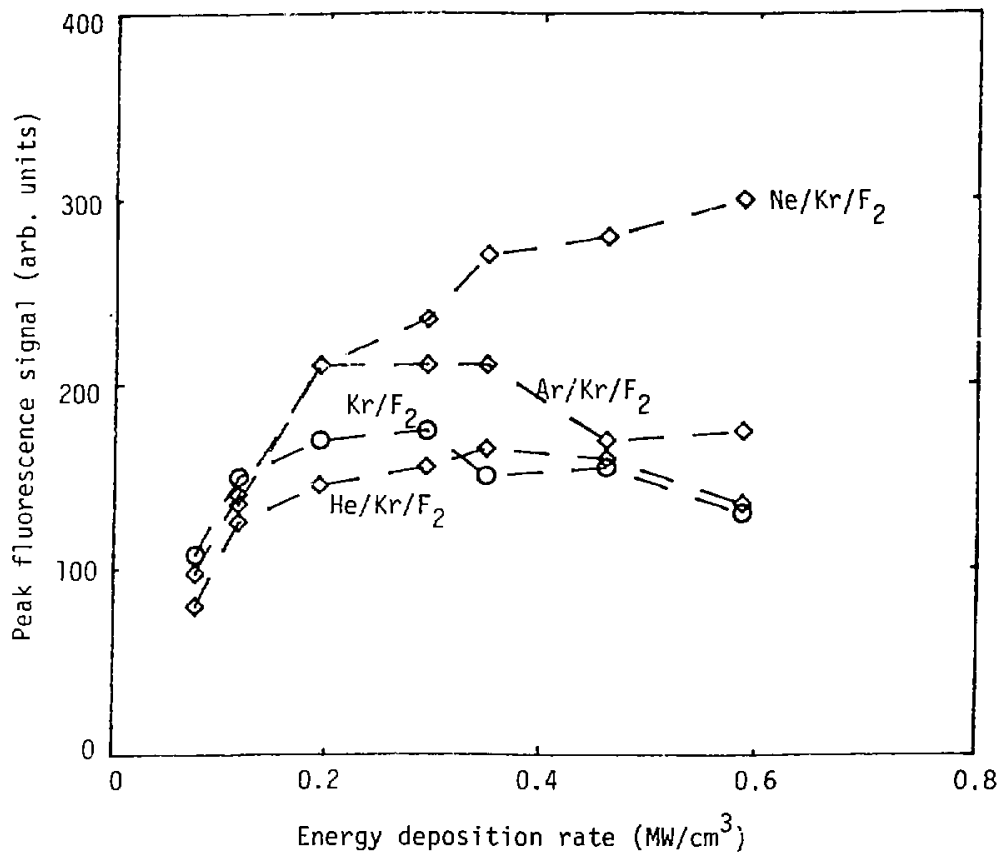

Fig. 1. Peak KrF* fluorescence signal vs average energy deposition rate for each of the gas mixtures described in the text at an average current density of $33 \mathrm{~A} / \mathrm{cm}^{2}$. 


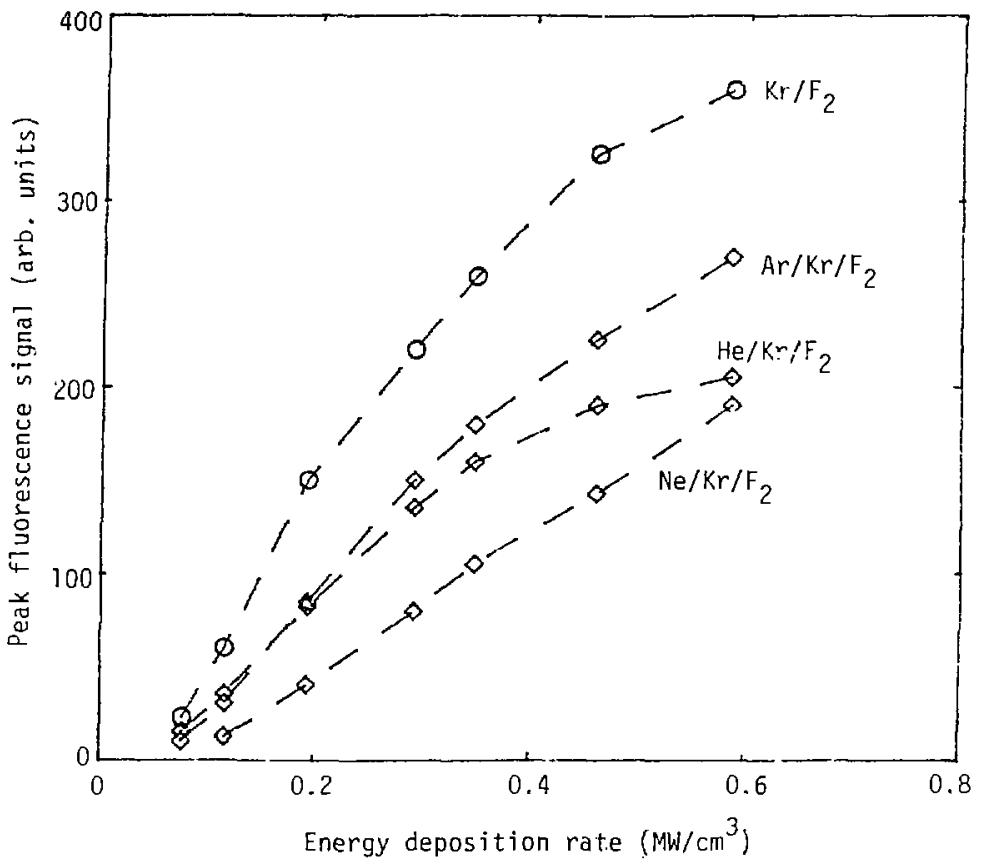

Fig. 2. Peak $\mathrm{Kr}_{2} \mathrm{~F}$ fluorescence signal vs average energy deposition rate for each of the gas mixtures described in the text at an average currerts density of $33 \mathrm{~A} / \mathrm{cm}^{2}$. 


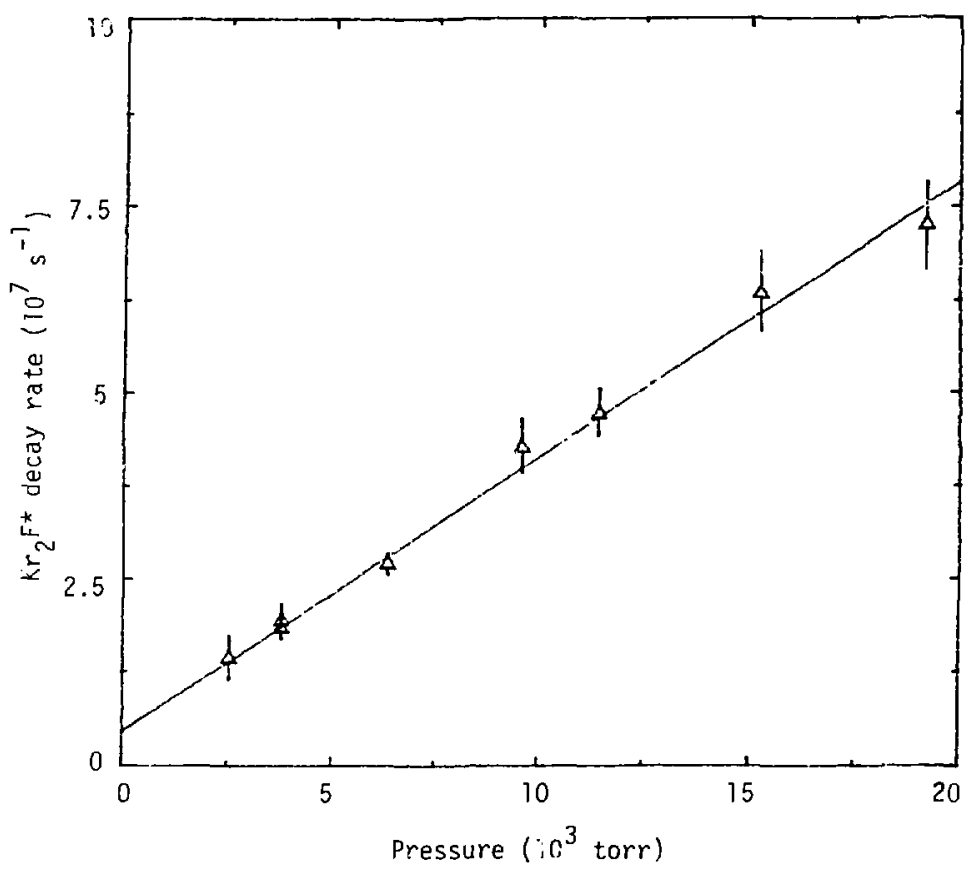

Fig. 3. $\operatorname{Kr}_{\frac{C}{6}}{ }^{*}$ decay rate vs gas pressure for the helium buffered mixture at an average current density of $33 \mathrm{~A} / \mathrm{cm}^{2}$. The solid line is the result of the 7 inear regression analysis described in the text. 


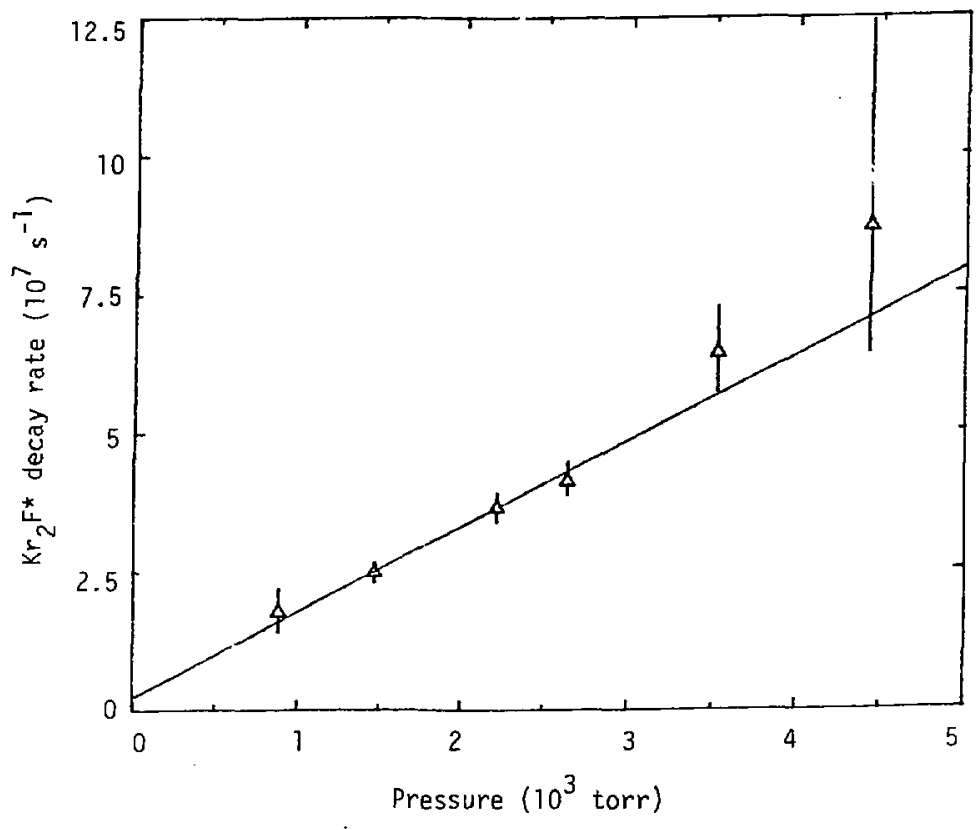

Fig. 4. $\mathrm{Kr}_{2} \mathrm{~F}^{*}$ decay rate vs gas pressure for the neon buffered gas mixture at an average current density of $33 \mathrm{~A} / \mathrm{cm}^{2}$. The solid line is the rasult of the linear regression analysis described in the text. 


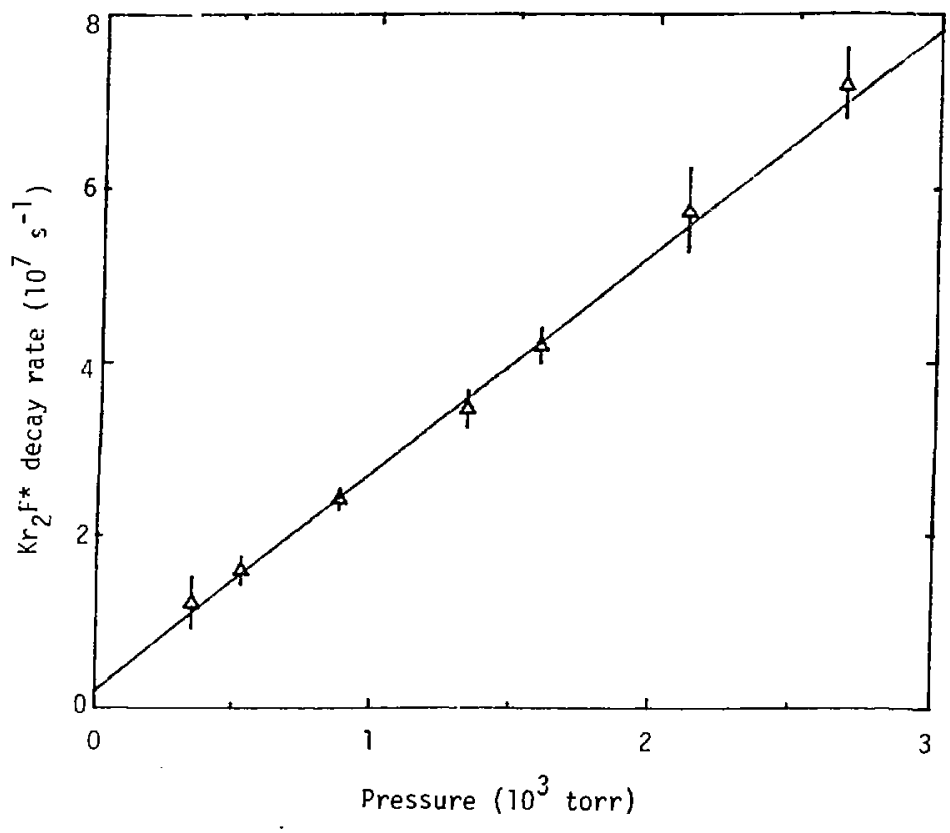

Fig. 5. $\mathrm{Kr}_{2}{ }^{\text {k* }}$ decay rate vs gas pressure for the argon buffered gas mixture at an average current density of $33 \mathrm{~A} / \mathrm{cm}^{2}$. The solid line is the result of the linear regression analysis described in the text. 


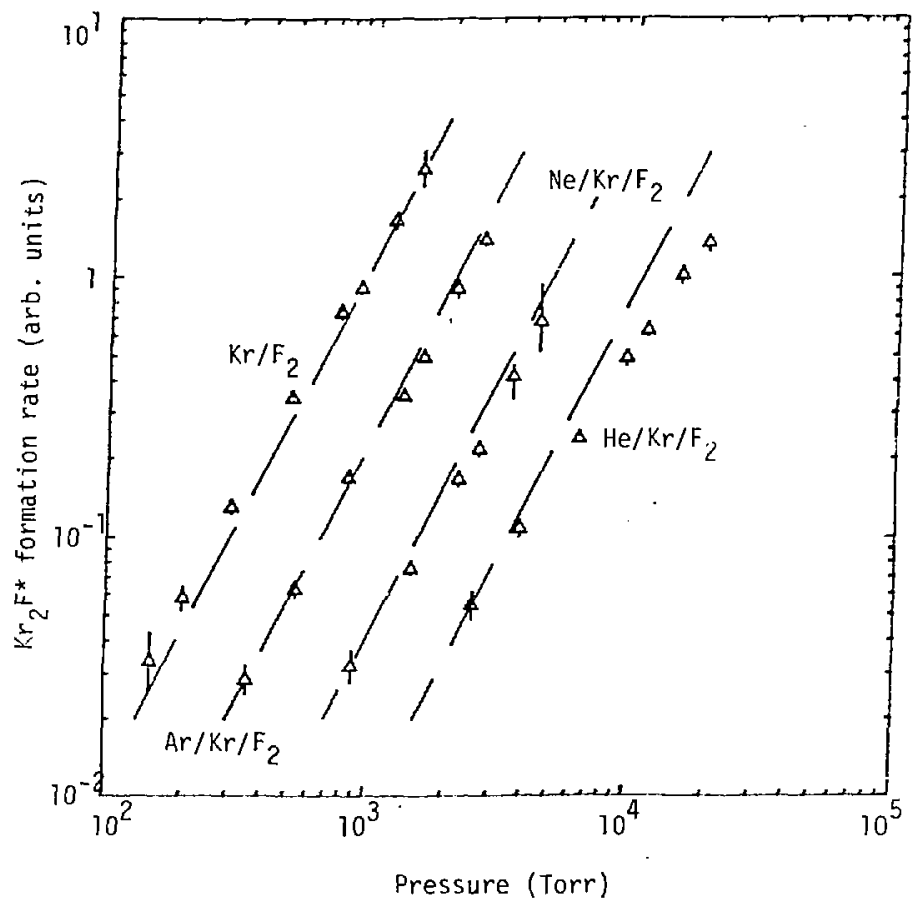

Fig. 6. $\mathrm{Kr}_{2} \mathrm{~F}^{*}$ formation rate vs gas pressure for each of the gas mixtures described in the text àt an average current density of $33 \mathrm{~A} / \mathrm{cm}^{2}$. 


\title{
Appenjix - Chapter $\underline{v}$
}

\author{
Quenching of $\mathrm{Ar}_{2} \mathrm{~F} *$ and $\mathrm{Kr}_{2} \mathrm{~F} *$ By Radiation, \\ Heavy Particles, and Electrons
}

K. S. Jancaitis and H. T. Powell

\section{Abstract}

Rare gas halide dimers (ArF* and $\mathrm{KrF}^{\star}$ ) and trimers $\left(\mathrm{Ar}_{2} \mathrm{~F}^{\star}\right.$ and $\left.\mathrm{Kr}_{2} \mathrm{~F}^{\star}\right)$ have heen studied using $50 \mathrm{~ns}, 600 \mathrm{keV}$, electron-beam excitation of two component gas mixtures. Fluorescence produced by excitation of either $\mathrm{Ar}$ or $\mathrm{Kr}$ mixed with either $\mathrm{F}_{2}, \mathrm{NF}_{3}$, or $\mathrm{SF}_{6}$ was studied as a function of e-beam current density $\left(2-500 \mathrm{~A} / \mathrm{cm}^{2}\right)$, gas pressure (200-2000 Torr), and mixture ratio. Trimer decay rates were determined by fitting the time dependence of trimer fluorescence to the solution of a single rate equation having a driving term proportional to the experimental dimer signal. Trimer lifetimes and collisional quenching coefficients have been determined at low current density $\left(<50 \mathrm{~A} / \mathrm{cm}^{2}\right)$ and electron quenching of the trimer has been studied at high current density. 


\section{Introduction}

Rare gas halide (RGH) trimer molecules such as $\mathrm{Ar}_{2} \mathrm{~F}^{*}$ and $\mathrm{Kr}_{2} \mathrm{~F}^{*}$ are important species in excimer laser media. ${ }^{1-6}$ RGH trimers are formed by three-body collisions of excited RGH dimers with rare gas atoms. This is a major loss process for the dimers and often produces for laser conditions a greater concentration of excited trimer than dimer molecules. In addition, as discussed in the following paper, $\mathrm{Ar}_{2} \mathrm{~F}^{*}$ and $\mathrm{Kr}_{2} \mathrm{~F}^{*}$ exhibit strong absorption bands in the $300 \mathrm{~nm}$ region which contribute tc the background optical absorption in tne excited medium at the $249 \mathrm{~nm}, \mathrm{KrF}$ laser wavelength. Recently, RGH trimers have also been shown to be interesting laser candidates themselves with the demonstration of laser action from $\mathrm{Kr}_{2} \mathrm{~F}^{\star}$ and $\mathrm{xe}_{2} \mathrm{Cl*}$.

RGH trimer emission occurs in broad (roughly $50 \mathrm{~nm}$ wide), unstructured bands approximately $2 \mathrm{eV}$ to the red of the main dimer transitions.' Emission from $\mathrm{Ar}_{2} \mathrm{~F}^{\star}$ is observed at $290 \mathrm{~nm}$ from mixtures of argon with fluorine atom donors excited by ar electron beam or discharge. The $A r_{2} F^{\star}$ emission increases with $A r$ pressure relative to the ArF* dimer emission at $193 \mathrm{~nm}$. The $\mathrm{kr}_{2} \mathrm{~F}^{\star}$ emission occurs at $410 \mathrm{~nm}$ and behaves similarly with respect to $\mathrm{Kr}$ pressure in relation to the $249 \mathrm{~nm}$ dimer emission. This pressure dependence as well as the observed quenching of the dimers in three-body callisions with rare gases ${ }^{2}$ suggest that the RGH trimers are formed collisionally trom the dimers. This view is supported by 
the similarity of their excited state structures--both dimers and trimers are ionically bound in their excited states (i.e., $\mathrm{Ar}^{+} \mathrm{F}^{-}$ and $\mathrm{Ar}_{2}^{+} \mathrm{F}^{-}$are good descriptions of the excited states). ${ }^{8-10}$

Although several autnors have suggested that trimers might be produced by reactions without involving a dimer precursor $1,11,12$ (e.s., $A r_{2}^{+}+F^{-}+M-A r_{2} F^{*}+M$ or $\left.A r_{2}^{*}+F_{2}-A r_{2} F^{*}+M\right)$, both theoretical arguments ${ }^{10}$ and experimental measurements ${ }^{2,3}$ suggest that such reactions are not dominant for usual conditions.

Previous studies have determined the radiative lifetimes of $\mathrm{Ar}_{2} \mathrm{~F}^{\star}$ and $\mathrm{Kr}_{2} \mathrm{~F}^{\star}$ and their quenching rates by some fluorine donors using the RGH fluorescence which followed short pulse excitation of gas mixtures by either proton beams, ${ }^{3}$ uv radiation, ${ }^{4}$ or electron beams. ${ }^{13}$ In this study, we have experimentally measured the radiative lifetimes of $\mathrm{Ar}_{2} \mathrm{~F}^{*}$ and $\mathrm{Kr}_{2} \mathrm{~F}^{*}$ and their quenching coefficients by $\mathrm{F}_{2}$ and $\mathrm{NF}_{3}$. We have also placed an upper limit on the trimer quenching coefficients by $\mathrm{SF}_{6}$. We have used $50 \mathrm{~ns}$ e-beam excitation of simple two-component mixtures of either $\mathrm{Ar}$ or $\mathrm{Kr}$ with $\mathrm{F}_{2}, \mathrm{NF}_{3}$, or $\mathrm{SF}_{6}$. We have simultaneously monitored both the aimer and triner fluorescence and have applied a unique analys is to determine trimer quenching rates. The observed dimer time dependence was utilizf $\mathrm{f}$ as a source term for trimer production in a simple rate equation describing the trimer. Solutions of the rate equation were compared to the experimental twimer time dependence to determine the trimer decay rate. In addition, to observe the effect of electron quenching we have studied the diner and tritner 
fluorescence over a large range of e-beam current densities, 5 to 500 $\mathrm{A} / \mathrm{cm}^{2}$. We find that electron quenching is a major factor in the trimer kinetics but produces for our conditions no obvious effect on the dimer. This difference is largely accounted for by the normally loriger decay time of the trimers $(\approx 50$ ns) compared to the dimers $(\approx 5 \mathrm{~ns})$ so that additional quenching by electrons causes a much larger relative effect for the trimers.

\section{Experimental Setup}

We have used a $600 \mathrm{keV}, 50$ ns-duration e-beam of variable current density to excite gas mixtures producing RGH fluorescence. The e-beam was powered by a Maxwell Pocobeam accelerator ${ }^{14}$ consisting of: a 28-stage Marx generator; a $10 \Omega$, water dielectric, pulse-forming line; a Sflf-breakdown output switch; and a field emission diode. A schematic diagram of the e-beam diode, beam-drift region, and laser cell is shown in Fig. 1. The pulsed output yoltage between the carbon cathode and the $1.3 \times 10^{-2}$ cm-thick, titanium diode foil was approximately $>\mathrm{MV}$ and the diode current was approximately $50 \mathrm{kA}$. The cross section of the e-beam was roughly $1 \times 10 \mathrm{~cm}^{2}$ at the diode foil.

Confined between two metal plates separated by $2.5 \mathrm{~cm}$, the beam propagated to the cell through a $15 \mathrm{~cm}$ long, beam-drift region. The gas pressure (air) in the beam drift region was 15 Torr. To reduce the beam current density, carbon attenuator plates (each $0.6 \mathrm{~cm}-\mathrm{ch}$ ick and perforated with numerous holes ranging from 0.1 to $1 \mathrm{~cm}$ diam) were 
individually placed midway in the drift region as shown schematically in fig. 1. As judged by chlorostyrene dosimetry at the cell the beam profile was quite uniform with the attenuators in place. Self-forces apparently cause the beam to homogenize within the drift region after passing through an attenuator plate. The electron beam finally passed through a foil support structure and $7.6 \times 10^{-3} \mathrm{~cm}$-thick Inconel foil to excite gas mixtures in the cell. Inconel was used as foil material because of $i$ ts resistance to fluarine attack. The support structure had a transmitting area of $2.5 \mathrm{~cm} \times 10 \mathrm{~cm}$ with transverse ribs to support the foil. The 14 ribs individually presented a width of 0.18 $\mathrm{cm}$ to the beam praducing a geometric transmission factor for the support structure of 75 percent.

We measured the e-beam current density impinging on the gas for the various attenuators by placing a Faraday cup in the gas cell immediately behind the cell foil. The current collector consisted of a $2.5 \mathrm{~cm} \times 10 \mathrm{~cm} \times 0.6 \mathrm{~cm}$ thick carbon plate a'ttached top and bottom along its $10 \mathrm{~cm}$ dimension to thin stainless steel sheets which provided a $13.6 \mathrm{~m} \Omega$ path to ground. To minimize inductance the stainless steel conductors were held firmly against a thin insulator separating them from the top and bottom surfaces of the cell at ground potential. The faraday cup was caliurated using a mercury-pulser-driven current source and its risetime was measured to be less than $10 \mathrm{~ns}$. 
The gas volume of the cell available for e-beam excitation was 10 $\mathrm{cm}$ long with a cross section of $2.5 \mathrm{~cm}$ by $2.5 \mathrm{~cm}$. However, to insure that our detectors viewed only fluorescence from a homogeneously-excited region, we placed a stainless steel insert in the cell to reduce the excited volume as shown schematically in fig. 1. The insert defined an excited volume having a length of $2.5 \mathrm{~cm}$, a height of $1 \mathrm{~cm}$, and a depth of $1.5 \mathrm{~cm}$. The e-beam excitation within this region was presumed to be uniform. We determined the current density impinging on this volume by removing the insart and using the Faraday cup with an appropriate metal mask.

Fluorescence was observed endwise through $1 \mathrm{~cm}$ diam holes in the insert block and transverseiy through a $0.3 \mathrm{~cm}$ diam hole. The hole diameters were made as small as nussible (consistent with adequate signal levels) compared to the depth of gas observed to minimize edge effects. We also chose the fluorescence volume to be small to minimize the effects of amplification and absorption on the observed fluorescence signais. This assumption was checked by comparing the shape of fluorescence signals from the $2.5 \mathrm{~cm}$ and $1 \mathrm{~cm}$ directions. At the nighest current densities, a small difference was found for the two dimer signals. To eliminate effects due to gain, the dimer flucrescence was measured in the $\mathrm{l} \mathrm{cm}$ direction for current densities greater than $200 \mathrm{~A} / \mathrm{cm}^{2}$. The gain and absorption coefficients described in the following paper also suggest the medium was optically-thin (when spectrally averaged over the emission bands) for these dimensions. 
For our excitation depth, gas pressures, and beam voltage (approximately $600 \mathrm{keV}$ in the cell), the energy deposition in the gas is simply proportional to current density since the percentage of the beam energy deposited in the gas is very small $(</ \%)$. The energy deposition was calculated for 1 and 2 atm of Ar using the three-dimensional deposition code SANDYL. ${ }^{15}$ The calculated depositions were approximately twice the values predicted by simply using the stopping powers tabulated by Berger and Selzer. ${ }^{16}$ This difference is largely caused by electron back-scatter from the stainless steel walls. Although the SANDYL-calculated deposition was roughly confirmed by pressure-rise measurements, we still regard our energy deposition values as somewhat uncertain. Our best estimate $(+25 \%)$ is that the deposited energy was $25 \mathrm{~mJ} / \mathrm{cm}^{3}$ per atmosphere of Ar at $100 \mathrm{~A} / \mathrm{cml}^{2}$ and $45 \mathrm{~mJ} / \mathrm{cm}^{3}$ per atmosphere of $\mathrm{Kr}$ at $100 \mathrm{~A} / \mathrm{cm}^{2}$.

The gas cell and its fill system, constructed of stainless steel, were thoroughly passivated with $F_{2}$ and evacuated between fills to less than $10^{-4}$ Torr. We measured subatmospheric pressures with a capacitance manometer and higher pressures with a strain gauge. Gas. mixtures were composed in 1 liter mixing bottles using commercially pure $F_{2}$ and $N F_{3}$ and research grade rare gases. To insure complete mixing the bottles were left several hours before use. We typically used gas from the same mixture batch for each data set and refilled the cell with fresh gas from the mixture bottle for each shot. 
Biplanar vacuum photodiodes, $2.5 \mathrm{~cm}$ in diameter, viewed the fluorescence from the excited volume through bandpass filters. The photodiodes were shielded by $1 \mathrm{~cm}$-thick lead to prevent $x$-ray induced signals. An S-20-photocathode detector monitored the trimer fluorescence while a CsTe-photocathode detector monitored the dimer fluorescence. We isolated the $\mathrm{Kr}_{2} \mathrm{~F}^{\star}$ fluorescence with a Corning 5-56 filter ${ }^{14}$ and the $\mathrm{Ar}_{2} \mathrm{~F}^{*}$ fluorescence with a Corning 7-54 filter. ${ }^{14}$ The detector response curves, filter transmissions, and trimer spectra are shown in the following paper (see Figs. 2 and 3 ). We isolated the $\mathrm{ArF}^{\star}$ and $\mathrm{KrF}{ }^{\star} \mathrm{B}-\mathrm{X}$ emissions with interference $f i l$ ters having bandwidths of $20 \mathrm{~nm}$ and $10 \mathrm{~nm}$ centered at 195 and $250 \mathrm{~nm}$, respectively. For absolute calibration purposes the photodiodes viewed the excited volume through pinholes as described in the following paper. However, for these studies the photodiodes were typically placed immediately behind the $\mathrm{MgF}_{2}$ output windows located approximately $5 \mathrm{~cm}$ from the excited volume.

The photodiode signals were monitored by Tektronix 7904 oscilloscopes ${ }^{14}$ housed in a screen room where the traces were photographed on a single-shot basis. The cable lengths for the two photodiodes were adjusted to be equal and a terminating resistor of $50 \Omega$ was used both at the detector and oscilloscope to eliminate reflected voltage signals. The risetime of the detertion system was approximately 1 ns. A saturating photodiode viewed light produced by the breakdown of the output switch and thus provided a trigger pulse. This signal was also split, differentiated, and delayed to provide a 
fiducial pulse. Since the timing of the e-beam pulse incident on the cell was fixed relative to the switch breakdown, this method permitted accurate relative timing of the signals to within 2 ns.

\section{Experimental Results}

A. Ar- $\mathrm{F}_{2}$ and $\mathrm{kr}-\mathrm{r}_{2}$ mixtures

The most exhaustive set of experiments was perfornied using $F_{2}$ as the fluorine donar. The dimer and trimer fluorescence signals were observed for independent variations in the electron beam current density, the total gas pressure, and the ratio of rare gas to $F_{2}$ partial pressures. The fluorescence signals from $A r-F_{2}$ mixtures were qualitatively similar to those from $\mathrm{Kr}_{-}-\mathrm{F}_{2}$ mixtures in all cases studied.

Comparisons of the e-beam current density, ArF* fluorescence, and $\operatorname{Ar}_{2} \mathrm{~F}^{*}$ fluorescence are shown in Fig. 2 for $10 \mathrm{w}\left(33 \mathrm{~A} / \mathrm{cm}^{2}\right)$ and high $\left(400 \mathrm{~A} / \mathrm{cm}^{2}\right)$ current density for a typical gas mixture and pressure. The negative pulse at the end of each trace is the common fiducial pulse. The current pulse was approximately rectangular and constant in shape as the current density was varied as shown in Fig. 2(a). Figure $2(b)$ shows that the ArF* signal followed the current pulse with about a $10 \mathrm{~ns}$ delay and was also roughly constant in shape. However, as shown in Fig. $2(\mathrm{C})$, the $\operatorname{Ar}_{2} \mathrm{~F}^{*}$ time dependence varied considerably with current density. 
The $A r_{2} F^{*}$ signai is unlike the ArF* signal mainly because of its longer radiative lifetime. At low current density the trimer signal appears to integrate the dimer signal and then decay with a time constant which is the same during and after the e-beam $\mathrm{Cl}$ ent pulse. This behavior is consistent with the view that the trimer is formed from the dimer by three-body collisions and then decays by a time-independent loss process consisting of radiative decay plus collisional quenching. At high current density, the trimer appears to approach a steady state more rapidly during the current pulse than it decays after the pulse. This suggests a larger quenching rate for the trimer during the pulse, presumably by electron collisions.

The peak fluorescence amplitudes plotted versus average current density also suggest an e-beam-related quenching of the trimers. Figures 3 and 4 show the peak $\mathrm{ArF}^{*}$ and $\mathrm{Ar}_{2} \mathrm{~F}^{\star}$ signals and peak $\mathrm{KrF}^{*}$ and $\mathrm{Kr}_{2} \mathrm{~F}^{*}$ signals, respectively, as a functior of average current density. While the dimer signals increase almost linearly with current density, the trimer signals roll-off strongly at the highest current densities. Because the trimer's normal decay time is much longer than the dimer's, electron quenching (or any other excitation-density dependent quenching) is comparatively more important for the trimer. Indeed to predict the trimer concentration accurately it appears necessary to include this additional quenching in a description of the trimer kinetics for current densities as low as $50 \mathrm{~A} / \mathrm{cm}^{2}$. Figures 3 and 4 also show that for these mixtures electron quenching does not cause a significant reduction in the dimer 
intensity for e-bearn current densities up to $500 \mathrm{~A} / \mathrm{cm}^{2}$. The same general behavior of the $\mathrm{KrF}^{\star}$ and $\mathrm{Kr}_{2} \mathrm{~F}^{\star}$ intensities with current density was also found for a typical KrF laser mixture consisting of 1500 Torr Ar, 100 Torr $\mathrm{Kr}$, and 5 Torr $F_{2}$.

$\therefore \mathrm{Ar}-\mathrm{NF}_{3}$ and $\mathrm{Kr}-\mathrm{NF}_{3}$ mixtures

Using $\mathrm{NF}_{3}$ as the fluorine donor, we varied the experimental parameters in much the same way as that described for $F_{2}$ mixtures. In both $\mathrm{Ar}-\mathrm{NF}_{3}$ and $\mathrm{K}:-\mathrm{NF}_{3}$ mixtures, the dimer fluorescence followed the e-beam current density in a manner similar to that observed for $F_{2}$ mixtures. The dimer fluorescence intensities using $\mathrm{NF}_{3}$ were roughly 40 percent of those using i2 mixtures. Although the behavior of the ii $_{2} F^{*}$ fluorescence was similar for $F_{2}$ and $\mathrm{NF}_{3}$ mixtures with argon, the $\mathrm{Kr}_{2} \mathrm{~F}^{*}$ behavior was radically different for $\mathrm{F}_{2}$ and $\mathrm{NF}_{3}$ mixtures with krypton.

The $\mathrm{Kr}_{2} \mathrm{~F}^{*}$ fluorescence signe's from a typical $\mathrm{Kr}-\mathrm{NF}_{3}$ gas mixture are shown in Fig. 5 for high and low current densities. At low current density the trimer decayed after the current pulse with a decay constant comparable to its radiative lifetime implying ihat $\mathrm{NF}_{3}$ collisional quenching of $\mathrm{Kr}_{2} \mathrm{~F}^{*}$ is small. At high current density the trimer decayed with a fast rate both during and after the current pulse. This suggests that the excitatiun-dependent quenching of $\mathrm{Kr}_{2} \mathrm{~F}^{\star}$ is more important and that its importance is relatively longer-lived for $\mathrm{NF}_{3}$ mixtures than for $F_{2}$ mixtures. Thus to determine the heavy particle quenching rate of $\mathrm{Kr}_{2} \mathrm{~F}^{*}$ by $\mathrm{NF}_{3}$ it 
was necessary to use very low current densities $\left(<10 \mathrm{~A} / \mathrm{cm}^{2}\right)$. To increase the fluorescence signals at these very low excitation rates, we generally removed the metal insert in the cell since we were not concerned about excitation uniformity or optical depth in that: case.

$$
\text { C. } \mathrm{Ar}_{-}-\mathrm{SF}_{6} \text { and } \mathrm{Kr}-\mathrm{SF}_{6} \text { mixtures }
$$

We found $\mathrm{SF}_{6}$ to be a relatively inefficient fluorine donor for producing RGH fluorescence. Although $\mathrm{SF}_{6}$ mixtures produced dimer signals similar in time dependence to either $\mathrm{F}_{2}$ or $\mathrm{NF}_{3}$ mixtures, the peak dimer intensities were less than 10 percent of the comparable $F_{2}$ case. The trimer signals were also smaller by a similar factor.

For both $\mathrm{Ar}-\mathrm{SF}_{6}$ and $\mathrm{Kr}-\mathrm{SF}_{6}$ mixtures the trimer fluorescence varied with current density much like the $\mathrm{Kr}^{-\mathrm{NF}_{3}}$ case. That is, there was a iong-lived excitation-dependent quenching. Thus to observe the heavy particle quenching rates of the trimer by $\mathrm{SF}_{6}$ we again used very low curren: densities $\left(<10 \mathrm{~A} / \mathrm{cm}^{2}\right)$. Tor these conditions both the $\mathrm{Ar}_{2} \mathrm{~F}^{*}$ and $\mathrm{Kr}_{2} \mathrm{~F}^{*}$ signals decayed in times comparable to their radiative lifetimes suggesting that trimer quenching by $\mathrm{SF}_{6}$ is quite small.

\section{Analysis}

The study of the trimer kinetics is considerably simpler than that for the dimer. First, the trimer decays on a relatively long time scale so that it may be easily observed after its production has 
ceased to determine its decay rate. Second, the dimer emission provides the time dependence of the source of trimer production. The dimer on the other hand is produced by several ionic recombination as well as neutral reaction processes which follow e-beam excitation. To analyze the trimer kinetics we made the following assumptions:

(i) That the dimer and trimer fluorescence signals are proportional to the corresponding species densities;

(ii) That the trimer is formed solely from the dimer, the formation rate being proportional to the dimer density;

(iii) That at low current densities the trimer is quenched with a time independent rate constant.

Under these assumptions at low current densities the trimer fluorescence signal $(Y(t))$ and the dimer fluorescence signal (W(t)) are related by a single equation

$$
\dot{Y}=\alpha w-\tau^{-1} Y
$$

the source term $\boldsymbol{\alpha}$ is given by

$$
\alpha=S_{Y} K / S_{W}
$$

where $k$ is the pressure dependent trimer formation rate constant, and $S_{W}$ and $S_{Y}$ are detector sensitivity factors defined by $W=$ $S_{W} n_{W}, Y={ }^{\prime}{ }^{n} n_{Y}$ where $n_{W}$ and $n_{Y}$ are the dimer and trimer number densities, respectively. The determination of these factors is discussed in detail in the Appendix. The decay term $\tau^{-1}$ in 
Eq. (1) is the sum of radiative decay $\left(T_{r a d}^{-1}\right)$ and two body quenching processes $\left(k_{i} n_{i}\right)$.

Using the dimer fluorescence as input, we fitted the observed trimer time dependence at low current densities to the solution of Eq. (1) to determine best fit values for $\boldsymbol{\alpha}$ and $\tau^{-1}$. The oscillograms of the fluorescence signals were first digitized at approximately 40 points chosen to allow full definitions of the curves. Quadratic interpolation yielded the "experimental" values $Y_{j}=Y\left(t_{i}\right)$ and $W_{i}=W\left(t_{j}\right)$ on a 1 ns-spaced mesh of points in time.

Before solving Eq. (1) for a prediction of the trimer flucrescence to be matched to the experimental results, it is convenient to define an auxiliary variable $U(t)=Y(t) / \alpha$, so that Eq. (1) becomes

$$
\dot{u}=W-\tau^{-1} U \text {. }
$$

Using the experimental values of the dimer fluorescence as input, Eq. (3) was integrated numerically to get the values $u_{j}$, the predicted value $U\left(t_{j}\right)$, as a function of the parameter $\tau^{-1}$ (see Appendix). Notice that we represent experimental fluorescence intensities by capital letters and predicted values by lower case letters. The predicted value of the trimer fluorescence signal $y_{i}$ is then given as a function of $\boldsymbol{\alpha}$ and $\tau^{-1}$ as $y_{i}=\boldsymbol{\alpha} u_{i}$. 
The best-fit values of the parameters $\alpha$ and $\tau^{-1}$ were then determined by minimizing the error

$$
\Delta^{2}=\sum_{i}^{\top}\left(Y_{i}-y_{i}\right)^{2}
$$

between the experimental and predicted values for the trimer fluorescence signal. Minimizing $\Delta^{2}$ with respect to $\alpha$ gives

$$
\boldsymbol{\alpha}=\sum_{i} u_{j} Y_{i} / \sum_{i} u_{i}^{2}
$$

Thus $\boldsymbol{\alpha}$ is a scale factor which relates the reduced variable $u_{j}$ to the predicted $y_{i}$ which best fits the experimental data.

Using Eq. (Al) in the Appendix and Eq. (5) to find $u_{i}$ and $\alpha$ we determined the error $\Delta^{2}$ associated with a given value of $\tau^{-1}$. The error was then minimized with respect to $\tau^{-1}$ using a modified Gauss-Newton method where the derivatives were calculated numerically using finite differencing. The error in the best-fit value of $\tau^{-1}$ was estimated as that increase (or decrease) required to increase the error sum $\Delta^{2}$ to twice its minimum value. The error in the best-fit value for $\boldsymbol{\alpha}$ was simpiy estimated to be that associated with the error range for $\tau^{-1}$.

Values for the trimer radiative lifteimes and quenching coefficients were obtained from the variations of the trimer decay 
rate $\tau^{-1}$ with total gas pressure and gas mix. Specifically, we assumed $\tau^{-1}$ to be of the form

$$
\tau^{-1}=\tau_{\text {rad }}^{-1}+k_{r} n_{r}+k_{d} n_{d}
$$

where the subscripts $r$ and $d$ imply a $r$ are gas or a lluurme dunor, respectively. Taking the decay rate data at two different mixture ratios, we used multiple linear regression on Eq. (6), weighting each point according to its error range, to determine the trimer radiative lifetime and its quenching coefficient by the rare gas and the fluorine donor.

The analysis of the trimer time depencence at high current densities was performed in a different manner because of the presence of an addicional current dependent quenching term. Defining the experimental trimer aid dimer fluorescence signals as in Eq. (2), we expect them to be related at high current density by

$$
\dot{Y}=\alpha W-\tau^{-1} Y-\gamma(t) Y
$$

Notice that we make no assumptions about the time dependence of the additional quenrhing labeled $\gamma(\mathrm{t})$ but we presume that the values of $\alpha$ and $\tau^{-1}$ determined at low current density to continue to apply. Notice that we make no assuinptions about the products of trimer decay. In fact the analysis would still be correct even if the trimer 
decay led to the production of dimers since we have used the experimental dimer signal as input.

The function $\gamma(t)$ was determined first by digitizing the fluorescence curves to yield the experimental points $Y_{j}$ and $W_{i}$, as was done for the low current density data. Numerical differentiation of the function $Y(t)$, followed by a subtracting out of the radiative and heavy particle decay rates then yielded the current dependent quenching term $\gamma(t)$ (see the Appendix for details).

V. Results and Interpretation

A. Low Current Density

At low e-beam current density, the agreement between the experimental $(Y(t))$ and model $(y(t))$ trimer fluorescence was extremely good. Figure 6 shows the results of analyzing the low current density data of Fig. 2. An excellent match between the model and experimient was generally obtained when the current density was low enough to make any excitation-dependent quenching contribution negligible.

The best-fit values of $\tau^{-1}$ obtained from the low-current analysis are plotted in Fig. 7 for $A r-F_{2}$ mixtures as a function of total gas pressure. Results are shown for two $\mathrm{Ar}-\mathrm{F}_{2}$ mixture ratios and for two e-beam current densities. Notice that for these law current densities the fitted decay rates do not vary with current density. The $\mathrm{Ar}_{-} \mathrm{NF}_{3}$ and $\mathrm{Kr}-\mathrm{F}_{2}$ results behaved similarly. The 
solid lines in Fig. 7 are the results of the multiple linear regression fit of the values of $\tau^{-7}$ to the form shown in Eq. (6). In this way we determined both the $\mathrm{Ar}_{2} \mathrm{~F}^{*}$ radiative lifetime and the rate coefficients for $\mathrm{Ar}_{2} \mathrm{~F}^{*}$ quenching by both $\mathrm{Ar}$ and $\mathrm{F}_{2}$. The results from fitting the $\mathrm{Kr}-\mathrm{F}_{2}$ and $\mathrm{Ar}-\mathrm{NF}_{3}$ data were generally similar to those shown in Figs. 6 and 7.

The best fit values of $\tau^{-1}$ for $\mathrm{Kr}_{2} \mathrm{~F}^{*}$ in $\mathrm{Kr}-\mathrm{NF}_{3}$ mixtures are shown in Fig. 8. The small quenching rate constant ior $\mathrm{Kr}_{2} \mathrm{~F}^{\star}$ by $\mathrm{NF}_{3}$ suggested in the discussion of the experimental results is evident by the slower increase in the quenching rate with gas pressure. The fitted trimer quenching rates for $\mathrm{SF}_{6}$ mixtures varied so slowly that there was no clear dependence on gas pressure in that case.

The trimer radiative lifetimes and quenching rate constants obtained in this manner are summarized in Tables $I$ and $: I$, along with the results of other workers for comparison. The radiative lifetimes are somewhat longer than both the calculated values and previous measurements. Our $F_{2}$ and $\mathrm{NF}_{3}$ quenching rate coefficients are in the range of several previous determinations. There appears to be no previous data for trimer quenching by $\mathrm{SF}_{6}$.

The values of the trimer formation rate constant $\boldsymbol{\alpha}$ determined by our fitting procedure are shown in Figs. 9 and 10 for $\operatorname{Ar}_{2} F^{\star}$ and $\mathrm{Kr}_{2} \mathrm{~F}^{*}$, respectively. Because of the large number of overlapping 
data points we have found it convenient in both figures to shift some of the data either up or down a factor of 2 in ,ressure. For comparison, we have associated with each data group a common line having a $p^{2}$-slope which has been similarly shifted in pressure. Representative error bars are shown for only one set of data points.

It is evident from Figs. 9 and 10 that the trimer formation rate is independent of fluorine donor, mixture ratio, and e-beam current density. The values of $\alpha$ obtained from $\mathrm{SF}_{6}$ mixtures were also consistent with this conclusion. This result is expected if the trimer is formed solely from the dimer and would not be expected for trimer formation via alternative mechanisms. Although we expect a simple $p^{2}$-dependence for a three-body formation rate, the curves for the various conditions all deviate from the $p^{2}-1$ ine at high pressures. Though we have no clear explanation of this effect it is consistont with a decrease in the effective radiative lifetime of the dimer at high pressure, as previously reported for $\mathrm{KrF}^{\star} .{ }^{4} \mathrm{~A}$ decrease in effective dimer lifetime might be caused by $B-C$ state mixing as well as vibrational relaxation since the radiative lifetime differs for the $B$ and $C$ states and for different vibrational levels. 17 :

Using the sensitivity factors described in the Appendix and $t q$. (2), one can convert the $p^{2}$ lines in Figs, 8 and 9 to three-body formation rate coefficients $k$ defined by $k=k n_{r}^{2}$. However, since the effective radiative lifetime of the dimer is uncertain, we leave 
it as a parameter $\tau_{d}$. Thus we obtain $k \tau_{d}$ products of $3.1 \times 10^{-39} \mathrm{~cm}^{6}$ for $\mathrm{Ar}_{2} \mathrm{~F}^{*}$ formation from $\mathrm{ArF}$ * in argon and $2.1 \times 10^{-39} \mathrm{~cm}^{6}$ for $\mathrm{Kr}_{2} \mathrm{~F}^{\star}$ formation from $\mathrm{KrF}^{\star}$ in krypton. These values have roughly a \pm 25 percent uncertainty resulting from the uncertainty in the sensitivity factors. The $k \tau_{d}$ product for argon is in the range of previous measurements. ${ }^{2,3,13}$ The value for krypton is somewhat smaller than previous determinations. ${ }^{2,3,18}$

\section{B. High Current Density}

Figure 11 shows the results of analyzing the high current data shown in Fig. 2 using the analysis described in Sect. IV. During the current pulse, the excitation-dependent quenching rate is about three times greater than the sum of the radiative and heavy particle quenching. In addition, this additional quenching goes to zero after the current pulse as expected for quenching by a transient species.

Figures 12 and 13 show characteristic values of the current-dependent quenching rates obtained by averaging over the current pulse for $A r-F_{2}$ and $\mathrm{Kr}_{2} \mathrm{~F}_{2}$ mixtures, respectively. Again, representative error bars are shown on only one set of data points in each figure. The error range shown in the average current density is the full range of the current variation over the pulse length. Each solid line is a linear fit to the data at a given gas mixture. Within the scatter of the data, the additional quenching increases linearly with current density as expected. However, it does not vary inversely with $F_{2}$ density as one would expect quenching by electrons to vary 
in a simple model discussed below. Although the current-dependent quenching generally declines with added $F_{2}$ the dependence is weak.

In a simple model when the primary electron loss is dissociative attachment to $F_{2}$, the electron density is given by the production rate of electron-ion pairs divided by the attachment rate to $F_{2}$. The production rate of electron-ion pairs can be determined from the e-beam energy deposition rate assuming an energy cost of 26.2 eV/electron-ion-pair in argon and $24.3 \mathrm{eV} / \mathrm{electron-ion-pair} \mathrm{in}$ krypton. ${ }^{19}$ Because the electron attachment rate coefficient by $F_{2}$ is very fast, approximately $5 \times 10^{-9} \mathrm{~cm}^{3} \mathrm{~s}^{-1}, 20$ the electron density should track the current pulse with a delay of less than 2 ns for our conditions. Presuming the electron attachment rate coefficient by $F_{2}$ to be constant, the electron density should vary inversely with $F_{2}$ density in this madel.

One coinplication to this model is that the addition of $F_{2}$ can cause a deviation in the electron energy distribution because of the large loss rate for elect ons near zero energy. 20 This could cause the effective attachment coefficierit referenced to the total electron density to decline. In principle this might be the cause of the behavior shown in Figs. 12 and 13. However, a clear resolution of this problem is not possible without an independent measurement of the electron density for these conditions. 
We can estimate the trimer quenching coefficient by electrons via the simple prescription above and the energy deposition values given in Sect. II. Using the data in Figs. 12 and 13 at 2.5 Torr of $F_{2}$ and assuming an electron attachment coefficient of $5 \times 10^{-9} \mathrm{~cm}^{3} \mathrm{~s}^{-1}$, we estimate the electron quenching rate coefficient of $\mathrm{Ar}_{2} \mathrm{~F}^{\star}$ is $4 \times 10^{-8} \mathrm{~cm}^{3} \mathrm{~s}^{-1}$ and that of $\mathrm{Kr}_{2} \mathrm{~F} *$ is $1.3 \times 10^{-7} \mathrm{~cm}^{3} \mathrm{~s}^{-1}$.

These quenching coefficients are similar in magnitude to those previously measured for $\mathrm{ArF}^{*}$ and $\mathrm{KrF}^{\star},{ }^{21}$ and estimated for $\mathrm{Ar}_{2} \mathrm{~F}^{*}{ }^{6}$ Because of their ionic structure, $\mathrm{RGH}$ trimirs may be quenched by a mechanism analagous to rare gas dimer ion recombination. However, because the trimers have no net charge, it is not surprising that these rate coefficients are significantly less than measured rare-gas, dimer-ion, recombination coefficients. ${ }^{22}$

We have also considered the possibility that the excitation-dependent quenching of the trimers might be caused by a transient species other than electrons. The trimers themselves are the transient species of greatest concentration $\left(\geqslant 5 \times 10^{15} \mathrm{~cm}^{-3}\right)$ as discussed in the following paper. To account for the addicional quenching in $F_{2}$ mixtures by trimer-trimer collisions would thus require a quenching coefficient of order $10^{-7} \mathrm{~cm}^{3} \mathrm{~s}^{-1}$. Such a value seems unreasonably large for a heavy-particle quenching rate. Attributing this quenching to any of the other short-lived species in the gas would require an even larger quenching coefficient. 
When either the initial fluorine donor quenching coefficient is small (such as for the $\mathrm{Kr}-\mathrm{NF}_{3}$ and rare gas-SF${ }_{6}$ mixtures), or the excitation of the gas is sufficient to deplete the fluorine donor, fragments produced from the fluorine donor could contribute significantly to the trimer decay rate. The simultaneous presence of quenching by both electrons and fragments complicates the interpretation of the results. Consider the $\mathrm{Kr}-\mathrm{NF}_{3}$ data shown in Fig. 8. If we assume that one fragment was produced per initial excitation in the gas and also assume that the current-dependent quenching at late times was entirely caused by fragments, it would require a fragment quenching coefficient on the order of $10^{-10} \mathrm{~cm}^{3} \mathrm{~s}^{-1}$. Hence, it seems quite reasonable in this case to attribute the late-time quenching to fragments.

In the highly-excited rare gas- $F_{2}$ mixtures at low $F_{2}$ pressures, a similar calculation indicates that nearly all of the $F_{2}$ was dissociated. However, we still found from the analys is that the trimer quenching rate after the current pulse corresponded closely to that expected from the initial $F_{2}$ density (see Fig. 11). This suggests that the quenching rate coefficient of the RGH trimers by fluorine atoms is between .5 and 1 times the corresponding value for $F_{2}$. The dissociation of $F_{2}$ could also effect the predicted electron densities and thus the interpretation of the data in Figs. 12 and 13 . 
V1. Conciusions

The time dependence and pressure dependerice of $\mathrm{Ar}_{2} \mathrm{~F}^{*}$ and $\mathrm{Kr}_{2} \mathrm{~F}^{*}$ fluorescence observed using e-beam excitation of Ar or $\mathrm{Kr}$ mixtures with either $\mathrm{F}_{2}, \mathrm{NF}_{3}$, or $\mathrm{SF}_{6}$ are consistent with trimer formation by three-body collisions of rare gases with the corresponding RGH dimer. At low excitation rates (e-beas current densities less than 50 $\mathrm{A} / \mathrm{cm}^{2}$ ) the trimer decay is usually dominated by radiative decay plus heavy particle collisional quenching. We have dete mined trimer decay rates by fitting the time dependence of trimer fluorescence to the solution of a rate equation having a driving term proportional to the experimental dimer signal. Stern-Volmer plats of the fitted rates have yielded trimer radiative lifetimes and collisional quenching coefficients by $\mathrm{F}_{2}$ and $\mathrm{NF}_{3}$. Quenching coefficients of the trimers by rare gases and $\mathrm{SF}_{6}$ are very small.

Excitation dependent quenching of the trimers is observed for e-beam current densities greater than $50 \mathrm{~A} / \mathrm{cm}^{2}$. This additional decay causes 3 change in the trimer amplitudes which is consisten with the observed change in Limer time dependence. For $F_{2}$ mixtures the excitation-dependent decay rate follows the current pulse suggesting that it is caused by a transient species. Based on the magnitude of this additional decay we are forced to conclude that it is caused by electrons even though the expected dependence on $F_{2}$ pressure is not found. In cases where the trimer quenching by the 
fluorine donor is small, trimer deactivation by dissociation iragments also appears to be sigrificant. 
Appendix to the Appendix - Chapter $\underline{V}$

Assuming that the dimer fluorescence signal is accurately approxinated by a linear function in any time interval $t_{j}$ to $t_{j+1}$, the solution of Eq. (3) for the predicted values $u_{i}$ in terms of the $h_{j}$ and $T^{-1}$ is given by the recursion relation

$$
\begin{aligned}
& u_{i+1}=u_{i} \exp (-\Delta t / \tau)+w_{i} \tau\left\{\frac{\tau}{\Delta t}[1-\exp (-\Delta t / \tau)]-\exp (-\Delta t / \tau)\right\} \\
& +W_{i+1} T\left\{\begin{array}{l}
i-\frac{T}{\Delta t}[1-\exp (-\Delta t / \tau)] \\
1
\end{array}\right\}
\end{aligned}
$$

with appropriate initial conditions where $\Delta t=t_{i+1}-t_{i}$.

Assuming that both the dimer and fluorescence signals can be approimated by linear functions in any time interval $t_{\mathbf{i}}$ to $t_{\mathbf{i}+1}$, and defining $t_{i}^{+}=\left(t_{j+]}+t_{i}\right) / 2$, the solution of Eq. (7) for $\gamma(t)$ is given by

$$
\gamma\left(t_{i}^{+}\right)=\alpha \frac{\left(W_{i+1}+W_{j}\right)}{\left(Y_{i+1}+Y_{i}\right)}-\frac{2}{\Delta t} \frac{\left(Y_{i+1}-Y_{j}\right)}{\left(Y_{i+1}+Y_{j}\right)}-\tau^{-l}
$$

wher $\geq$ again $\Delta t=t_{j+1}-t_{j}$.

The detector sensitivity factors $S_{Y}$ and $S_{W}$ were determined by measuring the absolute emission intensities of both the dimer and trimer for a well-defined geometry. Pinholes of radii $0.36 \mathrm{~cm}$ were located at the cell output window and at the detector. The pinholes 
were separated by $54 \mathrm{~cm}$. The projection of an illuminated field at the detector thraugh the pinholes demonstrated that the field of view through the $2.5 \mathrm{~cm}$ excited length was unobstructed. For this simple geometry the detector sensitivity factor $S_{Y}$ for the trimer fluorescence is given by

with the following definitions:

$$
S_{Y}=\frac{Y}{n_{Y}}=D R_{L} \frac{h \nu}{T_{r a d}}\left\{\frac{\pi r_{1}^{2} r_{2}^{2}}{4 d^{2}} L\right\}
$$

$$
\begin{aligned}
Y= & \text { trimer detector signal in volts } \\
n_{Y}= & \text { trimer density } \\
D \quad= & \text { detector response in } A / W \text { averaged over the } \\
& \text { emission band } \\
R_{L}= & \text { load resistance } \\
h \nu= & \text { quantum energy of the trimer fluorescence photon } \\
r_{1}, r_{2} & =\text { pintole radii } \\
L \quad= & \text { length of excited gas } \\
d \quad= & \text { pinhole separation. }
\end{aligned}
$$

A similar formula holds for the dimer fluorescence detector sensitivity $\mathrm{S}_{\mathbb{W}^{*}}$ The factor in parenthesis, an effective volume, can be derived by simple application of the brightness tr.aorem. ${ }^{23}$ The determination of the detector response will be discussed in detail in a future paper on optical absorption in e-beam excited rare gas and rare gas halide laser mixtures. 
Table I

$\mathrm{Ar}_{2} \mathrm{~F}^{*}$ Decay Constants

Radiative Lifetime

$$
\begin{array}{rlrl}
\tau_{\text {rad }} & =205 \pm 20 \mathrm{~ns} & \text { (this :ork) } \\
& =185 \pm 46 \mathrm{~ns} & \text { (Ref. 3) } \\
& =219 \pm 15 \mathrm{~ns} & & \text { (Ref. 13) } \\
& =132 \mathrm{~ns} & & \text { (Theory, Ref. 10) }
\end{array}
$$

Quenching Rate Coefficients

$$
\begin{aligned}
k_{\mathrm{Ar}} & <10^{-14} \mathrm{~cm}^{3} \mathrm{~s}^{-1} \text { (this work) } \\
& <10^{-14} \mathrm{~cm}^{3} \mathrm{~s}^{-1} \text { (Ref. 13) } \\
k_{F_{2}} & =(1.79 \pm .06) \times 10^{-10} \mathrm{~cm}^{-3} \mathrm{~s}^{-1} \text { (this work) } \\
& =(2.1 \pm .5) \times 10^{-10} \mathrm{~cm}^{3} \mathrm{~s}^{-1} \text { (Ref. 3) } \\
& =(2.05 \pm .06) \times 10^{-10} \mathrm{~cm}^{3} \mathrm{~s}^{-1} \text { (Ref. 13) } \\
& =4 \times 10^{-10} \mathrm{~cm}^{3} \mathrm{~s}^{-1}(\text { Ref. } 5) \\
\mathrm{k}_{\mathrm{NF}_{3}} & =(1.07 \pm .07) \times 10^{-10} \mathrm{~cm}^{3} \mathrm{~s}^{-1} \text { (this work) } \\
& =(1.23 \pm .05) \times 10^{-10} \mathrm{~cm}^{3} \mathrm{~s}^{-1} \text { (Ref. 13) } \\
\mathrm{k}_{\mathrm{SF}_{6}} & <10^{-13} \mathrm{~cm}^{3} \mathrm{~s}^{-1}(\mathrm{this} \mathrm{work})
\end{aligned}
$$




$$
\begin{gathered}
\text { Table II } \\
\mathrm{Kr}_{2} \mathrm{~F}^{\star} \text { Decay Constants }
\end{gathered}
$$

Radiative Lifetime

$$
\begin{aligned}
T_{\text {rad }} & =225 \pm 21 \text { ns (this work) } \\
& =165 \pm 41 \mathrm{~ns} \text { (Ref. 3) } \\
& =181 \pm 12 \mathrm{~ns} \text { (Ref. 4) } \\
& =200 \pm 18 \mathrm{~ns} \text { (Ref. 12) } \\
& =132 \mathrm{~ns} \text { (Theory, Ref. 10) }
\end{aligned}
$$

Quenchirg Rate Coefficients

$$
\begin{aligned}
k_{\mathrm{Kr}} & <10^{-13} \mathrm{~cm}^{3} \mathrm{~s}^{-1} \text { (this work) } \\
k_{F_{2}} & =(1.8 \pm 0.2) \times 10^{-10} \mathrm{~cm}^{3} \mathrm{~s}^{-1} \text { (this work) } \\
& =(3.40 \pm 8.5) \times 10^{-10} \mathrm{~cm}^{3} \mathrm{~s}^{-1} \text { (P.ef. 3) } \\
& =1.5 \times 10^{-10} \mathrm{~cm}^{3} \mathrm{~s}^{-1} \text { (Ref. s) } \\
k_{N F_{3}} & =(2.7 \pm 1.0) \times 10^{-12} \mathrm{~cm}^{3} \mathrm{~s}^{-1} \text { (this work) } \\
{ }_{K_{S F}} & <10^{-13} \mathrm{~cm}^{3} \mathrm{~s}^{-1} \text { (this work) }
\end{aligned}
$$




\section{Figure Captions - Appendix}

Fig. I Schematic diagram of experimental setup for RGH trimer kinetics studies.

rig. 2 Comparison of the time dependence at low and high current densities of the (a) e-beam current, (b) ArF* fluorescence, and (c) $\mathrm{Ar}_{2} \mathrm{~F}^{*}$ fluorescence. $\therefore 2.5$ Torr $\mathrm{F}_{2}-1500$ Torr $\mathrm{Ar}$ gas mixture was used.

Fig. 3 Peak ArF* and $\operatorname{Ar}_{2} F^{\star}$ fluorescence signals vs average e-beam current density for a 5 Torr $\mathrm{F}_{2}-1500$ Torr Ar gas mixture.

Fig. 4 Peak $\mathrm{KrF}^{*}$ and $\mathrm{Kr}_{2} \mathrm{~F}^{\star}$ fluorescence signals vs average e-beam current density for a 5 Torr $F_{2}-750$ Torr $\mathrm{kr}$ gas mixture.

Fig. 5 Comparison of the tise dependence of the $\mathrm{Kr}_{2} \mathrm{~F} * \mathrm{fluorescence}$ at low and high current densities for a 5 Torr $\mathrm{NF}_{3}-750$ Tor: $\mathrm{Kr}$ gas mixture. The average current densities were 2.2 $\mathrm{A} / \mathrm{cm}^{2}$ and $400 \mathrm{~A} / \mathrm{cm}^{2}$.

Fig. 6 Comparison of the experimental and model trimer fluorescence signals at low current density Curve "E" is the experimental ArF* signal of Fig. 2(c), curve "T" the result of the analys is of Sect. IV.

Fig. $7 \operatorname{Ar}_{2} F^{*}$ decay rate vs gas pressure for concentration ratios of (a) $[\mathrm{Ar}] /\left[\mathrm{F}_{2}\right]=300 / 1,(\mathrm{~b})[\mathrm{Ar}] /\left[\mathrm{F}_{2}\right]=600 / 1$. The solid lines are the results of the linear regression analysis described in the text. 
Fig. $8 \mathrm{Kr}_{2} \mathrm{~F}^{*}$ decay 'ate vs gas pressure for concentration ratios of (a) $[\mathrm{Kr}] /\left[\mathrm{NF}_{3}\right]=75 / 1$ and (b) $[\mathrm{Kr}] /\left[\mathrm{NF}_{3}\right]=150 / 1$. The solid lines are the results of the linear regression analysis described in the text.

Fig. $9 \mathrm{Ar}_{2} \mathrm{~F}^{*}$ formation rate $\alpha$ vs gas pressure for various gas mixtures and e-beam current densities: (a) $A r-F_{2}, 11$ $\mathrm{A} / \mathrm{cm}^{2}, \mathrm{X}=0.5$; (b) $\mathrm{Ar}-\mathrm{F}_{2}, 33 \mathrm{~A} / \mathrm{cm}^{2}, \mathrm{X}=1$; (c) $\mathrm{Ar}-\mathrm{NF}_{3}, \| \mathrm{A} / \mathrm{Cm}^{2}, X=2$. The triangles and circles are results for mixtures with concentration ratios of argon to fluorine donor of $300 / 1$ and $600 / 1$, respectively.

Fig. $10 \mathrm{Kr}_{2} F^{*}$ formation rate $\boldsymbol{\alpha}$ vs gas pressure for various gas mixtures and e-beam current densities: (a) $\mathrm{Kr}-\mathrm{F}_{2}, 11$ $\mathrm{A} / \mathrm{cm}^{2}, X=0.5$, (b) $\mathrm{Kr}-\mathrm{F}_{2}, 33 \mathrm{~A} / \mathrm{cm}^{2}, X=1$, (c) $\mathrm{Kr}-\mathrm{NF}_{3}, 2.2 \mathrm{~A} / \mathrm{cm}^{2}, \mathrm{X}=2$. For (a) and (b) the triangles and circles are results for mixtures with concentration ratios of $[\mathrm{Kr}] /\left[\mathrm{F}_{2}\right]$ of $150 / 1$ and $300 / 1$, respectively. For (c) the triangles and circles are res::? ir for mixtures with concentration ratios of $[\mathrm{Kr}] /\left[\mathrm{NF}_{3}\right]$ of $75 / 1$ and $150 / 1$, respectively.

Fig. $11 \mathrm{Ar}_{2} \mathrm{~F}^{*}$ quenching rates vs time obtained from analysis of the high current density data of Fig. 2. The sum of the radiative and heavy particle decay rates is $\tau^{-1}$; the excitation-dependent quenching rate is $\boldsymbol{\gamma}(t)$. 
Fig. 12 Current dependent $\mathrm{Ar}_{2} \mathrm{~F}^{*}$ quenching rate vs current density for gas mixtures of: (a) 2.5 Torr $F_{2}-1500$ Torr Ar (A); (b) 5.0 Torr $F_{2}-1500$ Torr $A r(O)$; (c) 7.5 Torr $F_{2}-1500$ Torr Ar (0). The solid lines are least square fits to the data for the given gas mixtures.

Fig. 13 Current dependent $\mathrm{Kr}_{2} \mathrm{~F}^{*}$ quenching rate vs current density for gas mixtures of: (a) 2.5 forr $F_{2}-750 \operatorname{Turr} \mathrm{Kr}(\Delta)$;

(b) 5.0 Torr $F_{2}-750$ Torr $\mathrm{Kr}(D)$; (c) 7.5 Torr $F_{2}-750$ Torr $\mathrm{Kr}(0)$. The solid lines are least squares fit to the data for the given gas mixtures. 

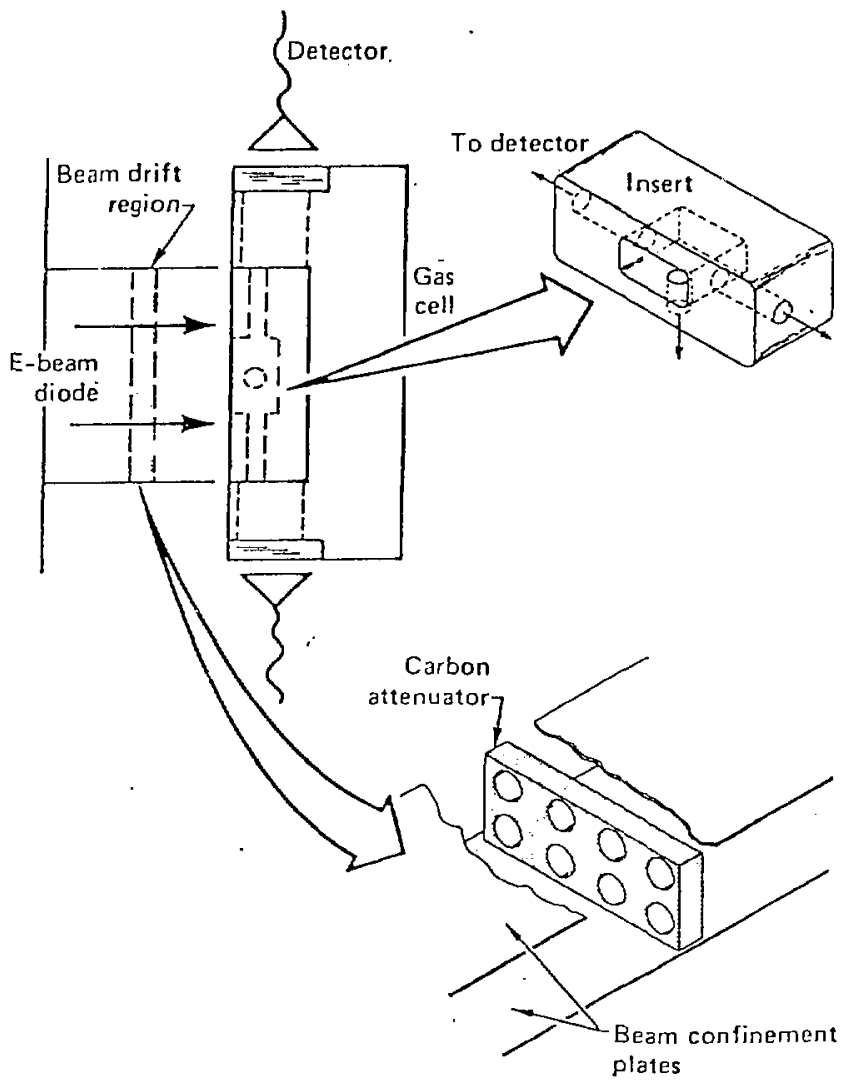

Fig. 1 

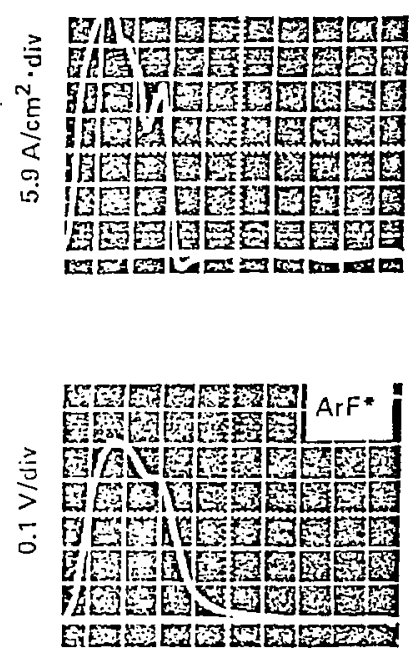

(a)

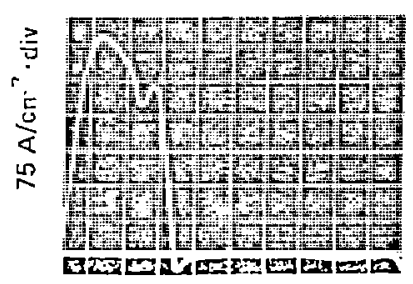

(b)

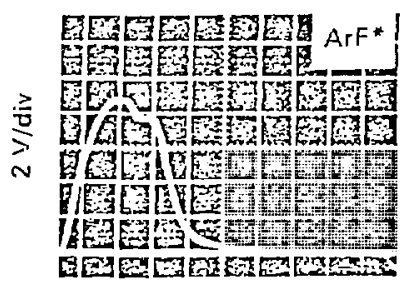

(c)

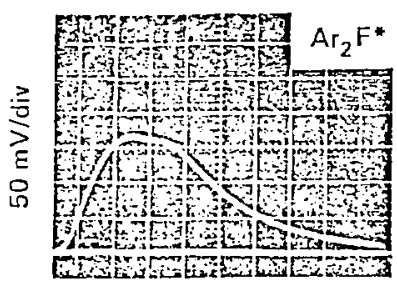

Time $(20 \mathrm{~ns} / \mathrm{div}$ )

Fig. 2 


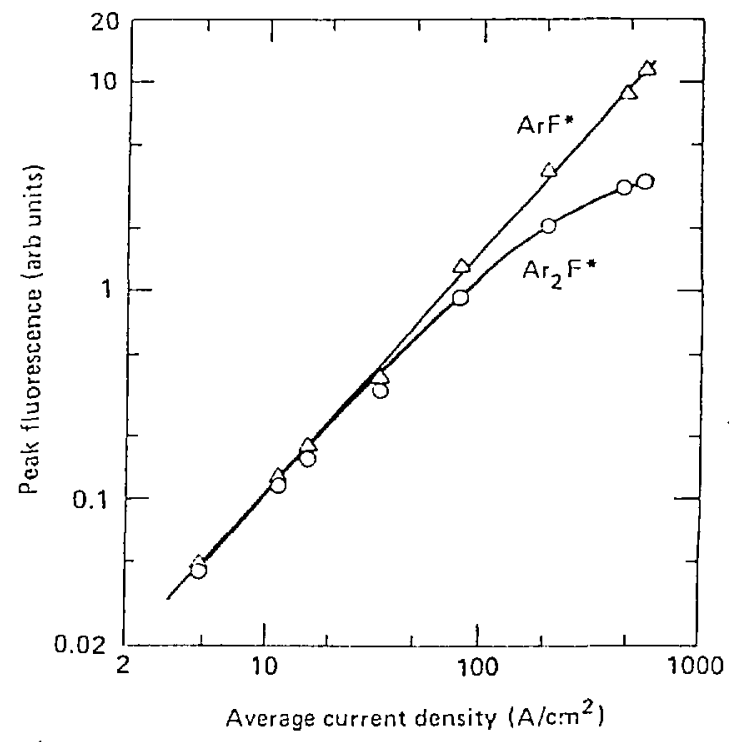

Fig. 3 


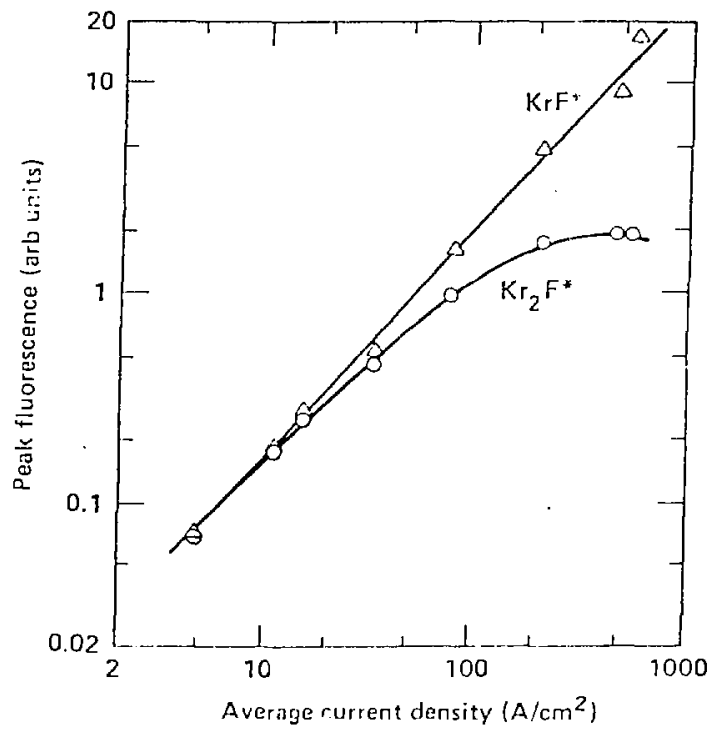

Fig. 4 
Low Current

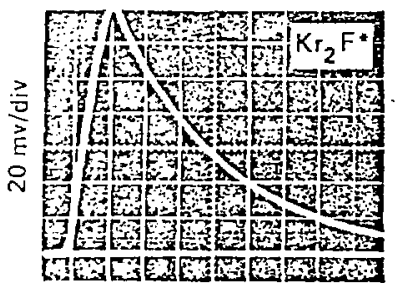

High Current

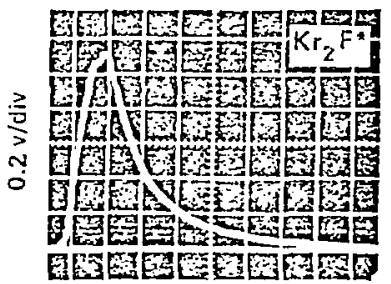

Time (50 ns/div)

Fig. 5 


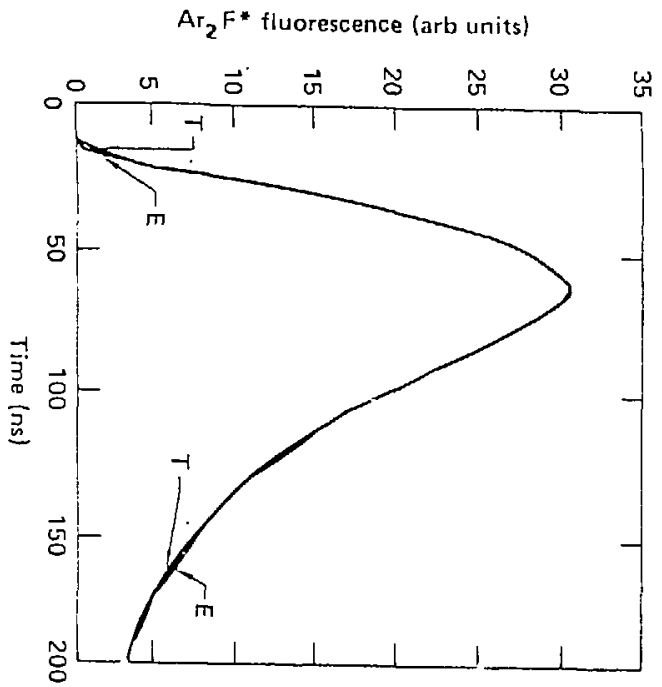




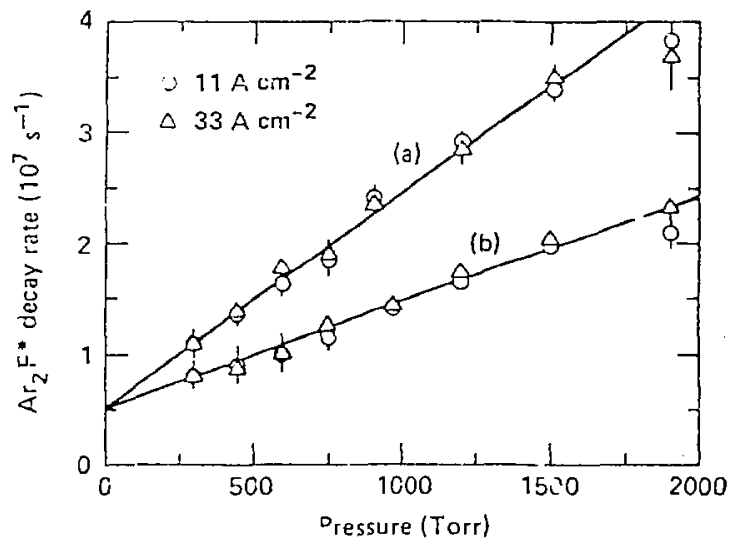

Fig. 7 


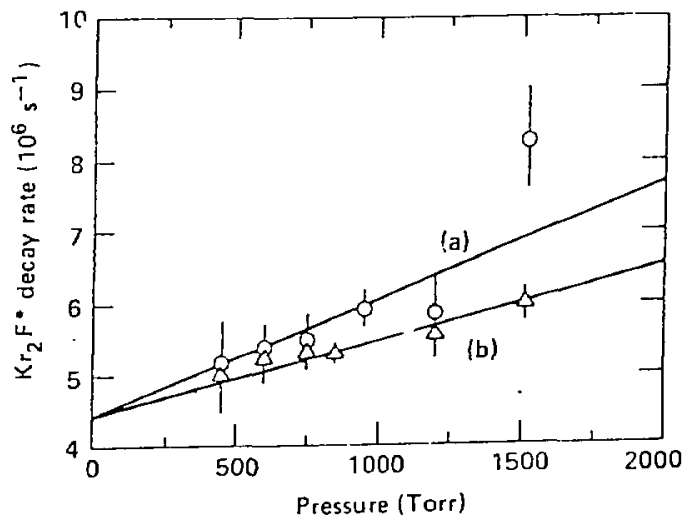

Fig. 8 


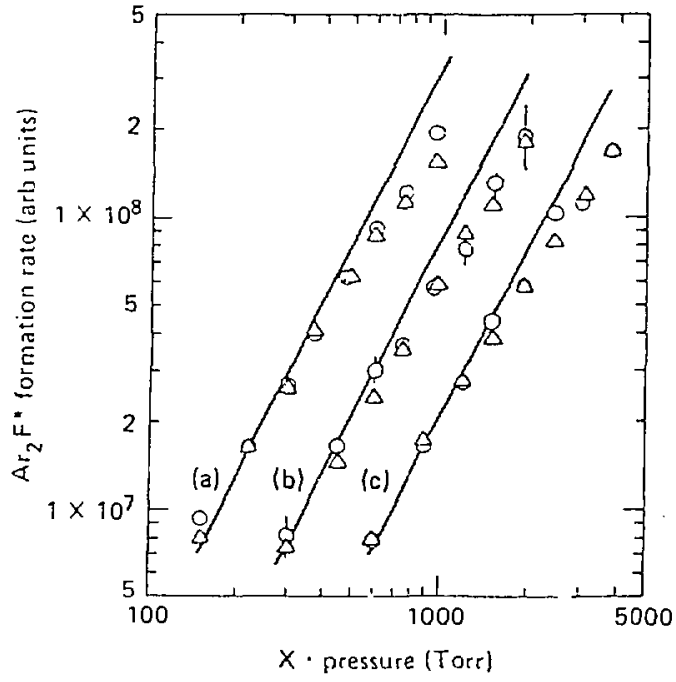

Fig. 9 


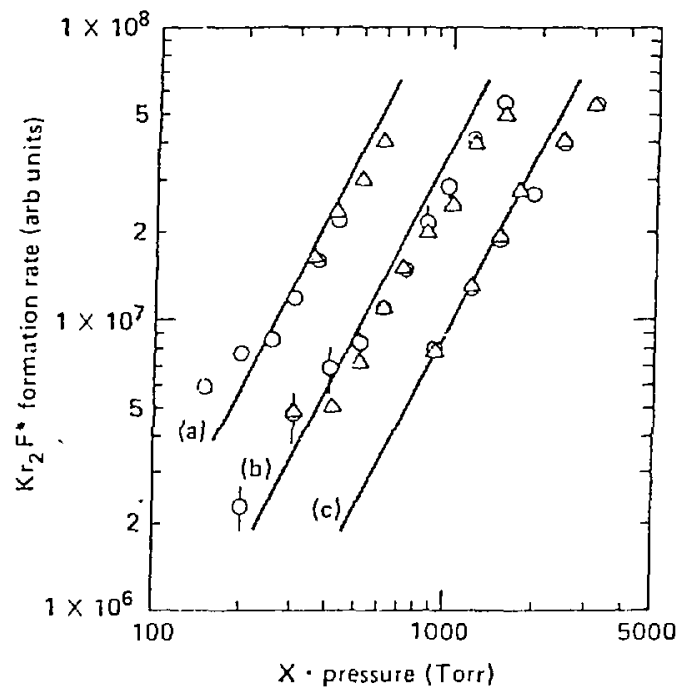

Fig. 10 
2
$=$
$=$

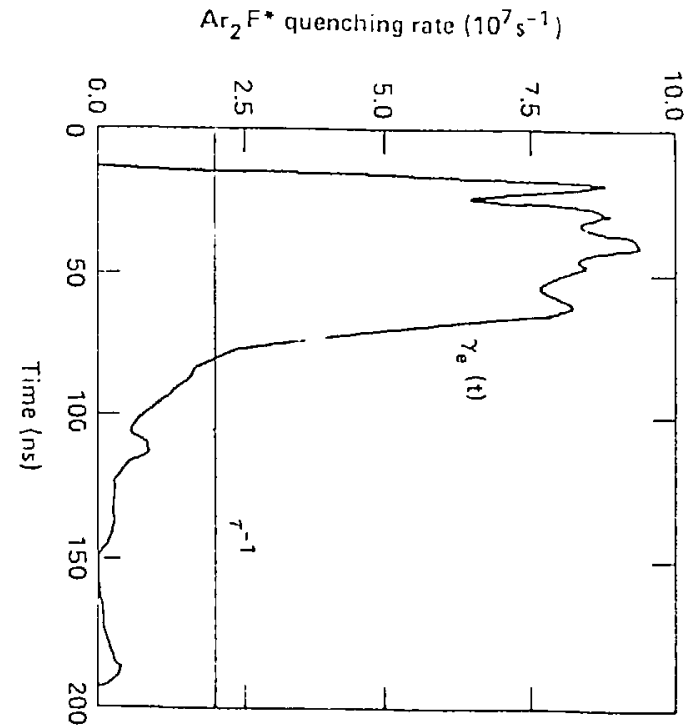




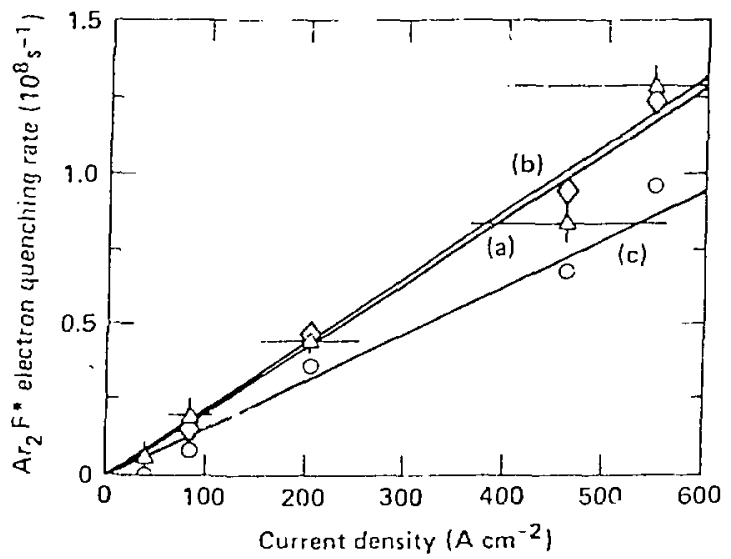

Fig. 12 


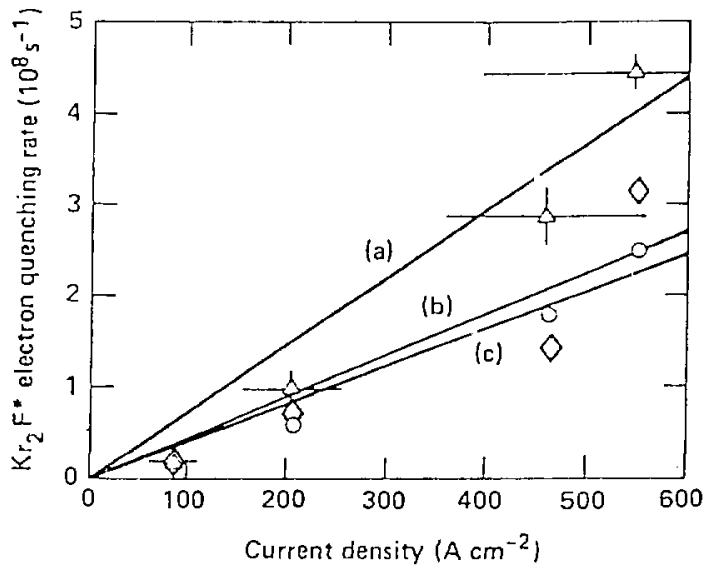

.Fig. 13 
R.eferences - Chapter $\underline{\underline{V}}$

I. D. C. Lorents, D. L. Heustis, M. V. McCusker, H. H. Nakano, and R. M. Hil1, J. Chem. Phys. 68, 4657, (1978).

2. M. Rokni, J. H. Jacobs, and J. A. Mangano, Fhys. Rev. A16, 2216, (1977).

3. Chung-Hsuan Chen and M. G. Payne, IEEE J. Quant. Elect. QE-15, 149, (1979).

4. G. P. Quigley and W. M. Hughes, Appl. Phys. Lett, 32, 627, (1978).

5. W. L. Morgan and A. Szoke, Phys. Rev. A23, 1256, (1987).

6. D. E. Klimek, J. C. Hsia, J. H. Jacob, D. W. Trainor, C. Duzy, and H. A. Hyman, IEEE J, Quant. Elect. QE-17, 1847, (1981).

7. F. H. Tittel, G. Marowsky, W. L. Wilson, Jr., and M. C. Smayling, IEEE J. Quant. Elect. QE-17, 2268, (1981).

8. M. Krauss, J. Chem. Phys. 67, 712, (1977).

9. H. H. Michels, R. H. Hobbs, and L. A. Wright, Chem. Phys. Lett. 48, 158, 1977).

10. W. R. Wadt nd P. J. Hay, J. Chem. Phys. 68, 3850, (1978).

11. W. B. Lacina and D. B. Cohn, Appi. Phys. Lett. 32, 106, (1978).

12. A. Luches, V. Nassisi, A. Perrone, and M. R. Perrone, Opt. Comrn. 39, 307, (1981).

13. G. Marawsky, G. P. Glass, F. K. Tittel, K. Hola, W. L. Wilson, and H. Weber, IEEE J. Quant. Elect. (to be published) 
14. Reference to a company or product name does not imply approval or recommendation of the product by the University of California or The U. S. Department of Energy to the exclusion of others that may be suitable.

15. H. H. Colbert, SANDYL, Sandia Nat. Lab., Livermore, CA, SCL-DR-;20109 (1973).

16. N. J. Berger and S. M. Selzer, "Studies in Penetration of Charged Particles in Matter," Nuclear Science Series Rep. \#10, NAS-NRC Pub 1133 (Nat. Acad, of Science, Washington, D.C. 1964).

17. P. J. Hay and T. H. Dunning, Jr., J. Chem. Phys. 66, 1306, (1977).

18. J. G. Eden, R. W. Waynant, S. K. Searles, and R. Burinan, Appl. Phys. Lett. 32, 733, (1978).

19. D. C. Lorents, Physica 82C, 19, (1976).

20. Numerous measurements and calculations of the electron attachment rates by $F_{2}$ are reviewed in $A$. U. Hazi, A. E. Orel, and T. N. Rescigno, Phys. Rev. Lett. 46, 918, (7981).

21. D. W. Trainor and J. H. Jacob, Appl. Phys. Lett. 37, 675, (1980).

22. F. J. Mehr and M. A. Biondi, Phys. Rev. 176, 322 (1968).

23. Laser Proyram Annual Report - 1975, UCRL-50021-75, p. 500. 


\section{Chapter VI}

The Observation and Modelling of

KrF Laser fiction

\section{f. Introduction}

The previous five chapters have described the theoretical and experimental development of a kinetic model for the KrF laser system, including soine comparisons of the model to the results of both fluorescence and absorption taneriments. The acid est of this model however rests in the comparison of its predictions with the experimental observation. of laser operation to which it speci'ically applies. In this chapter a present the results of some experiments on the variation of the performance nf a KrF laser with gas mixture, and compare them with the predictions of our kinetics model.

In Section B. We describe the experimental setup used to produce and observe KrF laser action in e-beam excited gas mixtures. With the addition of U. V. mirrors, the gas cell described in Chaster $V$ and used in that case for the generation of rare gas halide excimer fluorescence was converted into a laser cavity. Both the time dependence of the laser flux and the total energy outpul from the 
laser were monitored. In addition, the time dependence of either the $\mathrm{KrF}^{\star}$ or $\mathrm{Kr}_{2} \mathrm{~F}^{\star}$ sidelight fluorescence during the laser pulse could be measured.

In the next section we describe the specific form of the kinetic model which we used to nimerically calculate the predicted behavior for such a laser system. Specifically we discuss the complications introduced when the laser flux has to "boot-strap" itself up from the spontaneous emiscion of the medium. In cases where the time required for this buildup is longer than the excitiation pulse it may happen that no measurable laser action occurs, even though the net gain of the cavity is always positive.

In Section 0 . we present our experimf results for the 3tion with gas mixture of the performa.... of e-beam pumped $\mathrm{Kr}-\mathrm{F}$ ? iwser gas mises buffered with either helium or neon at both high i-430 $\left.\mathrm{A} / \mathrm{cm}^{2}\right)$ and intermediate current density $\left(=160 \mathrm{~A} / \mathrm{cm}^{2}\right)$ and compare them with the predictions of our kinetics model. The exporimental and calculated values of both the total energy output from the laser and the time dependence of the laser flux were foutua to agree moderately well.

In Section $\varepsilon$. we conclude with a discussion of what has been learned in the development of our krF laser kinetics model. We list both the successes and the shortcomings of the present model, and poirst out the systems for which we feel it provides accurate predictions. Finally, we discuss some ways in which the 1 . del itself 
might be improved, especially as concerns the heavy particle kinetics of helium buffered mixtures.

\section{B. Experimental Technique}

A schematic diagram of the apparatus used to produce and obcerve KrF laser action is presented in Fig. ' The setup is essentially the same as that i'sed for the fluorescence experuments of Chapter $V$ and described in Section II of the appendix to that chanter. Modifications were made only in the configuration of the gas cell and in the diagnostic equipment used.

The length of the active volume of the cell (defined by the stainless steel insert) was increased to 10. cri. while its defth and height were held at 1.5 and $1.0 \mathrm{~cm}$., respectively. This change allowed us to make use of the full lateral extent of the electron beam and thus provided the maximum possible small signal gain in the medium. The spatially averaged current density injected into tr.is volume was measured with a suitably apertured faraday cup, and was found to be only slightly smalier $(<10 \%)$ than that observed for the $2.5 \mathrm{~cm}$. long volume discussed in Chapter $V$.

The laser cavity was defined by placing circular front surface Uielectric coated (on a $\mathrm{MgF}_{2}$ suistrate) U. V. mirrors at both ends of the 10. cin. long active region. The separation of the mirror surfaces from the lasing volume was small enough $(<1 \mathrm{~cm}$.) so that the 
absorption due to $F_{2}$ in this dead region was negligible $(<1 \%)$. At one end (facing a U. V. photodiode) we used a maximum-reflectivity mirror (reflectivity $99 \%$ at $249 \mathrm{~nm}$.) throughout the experiment. At the other end of the cavity we used a $50 \%$ reflective mirror for the measurements with argon as the buffer gas; with helium as the buffer a 70\% reflective mirror was used.

The active surfaces of the mirrors which faced the laser medium were concave with a 1 meter radius of curvature. The curved surfaces were aligned by superimposing their reflections of a helium neon laser beam directed down the center of the laser cavity with the incoming alignment bealn itself. As noted in Fig. 1., the area of mirrors (each having a diameter of $2.7 \mathrm{~cm}$.$) was considerably larger than the$ $.8 \mathrm{~cm}^{2}$ cross section of the output beam defined by the cell insert and the mirror edga. The off-axis mounting chosen allowed the laser to "see" less than one-fourth of the mirror area, and rotating the mirror mounts by $90^{\circ}$ made a previously unexposed section of the mirrors available. The mirrors were rotated and re-aligned after each data set was completed (typically after about 25 shots); though the active regions of the mirrors appeared to be degraded when viewed in the visible after such use there was no apparent drop-of ${ }^{*}$ in their U. V. periormance

The time dependence of the $\mathrm{KrF}$ laser signal was monitored using a CsTe-photocathode biplanar vacuum photodiode, $2.5 \mathrm{~cm}$ in diameter. Even though 7ess than $1 \%$ of the intra-cavity flux was transnitted by 
the mirror in the direction of the diode, the introduction of neutral density filters into the beam path was required to reduce the incident intensity to a level at which the diode was not saturated.

The energy output through the partially transmitting mirror at the other end of the cavity was measured using a Scientech Model 3602 laser power meter.' Since the duratio: of the laser pulse $(=50 \mathrm{~ns}$. was wery much smaller than the characteristic cooling time ( $)$ s.) of the active area of the power meter, the output signal from its thermocouple (monitored on a chart recorder), extrapolated to the moment of the laser pulse, was directly proportional to the total energy in the output beam.

The power meter was calibrated for energy measurement by comparing its output signal to that from a specially constructed volume calorimeter for a set of identical input pulses at $249 \mathrm{~nm} .^{2}$ In the latter case, the optical energy was incident on a thermally isolated cylinder of Pyrex $7740 \mathrm{glass,} \mathrm{whose} \mathrm{thickness} \mathrm{(}<1 \mathrm{~mm}$. ) was sufficient to completely absorb the input beam. Its temperature increase was then measured (relative to a similar nearby reference piece of glass to minimize the effects of thermal drift); coupled irith the specific heat of the sample thi then determined the energy of each calibration pulse. The power meter was used in these experiments, because, once calibrated, it had much more sensitivity than the volume calorimeter (9.3 mViJ as opposed to $95 \mu \mathrm{V} / \mathrm{J})$. 
The photodiode detector and laser power meter were aligned with the laser axis using the same helium neon laser beam as was used to position the cavity mirrors. In addition, the spaijal extent of the laser output beam in the direction of the power meter was determined by piaring Dylux paper in the beam path, ${ }^{1,3}$ and was found to be completely encompassed by the entrance aferture of the meter which insured that all of the output energy was captured.

The sidelight fluorescence from the center of the active volume of the cavity was monitored using a S-20 photodiode detector, whose line of sight into the gas cell was apertured to prevent any laser photons scattered off the sides of the exit hole from entering. Either the $\mathrm{KrF}^{\star}$ or the $\mathrm{Kr}_{2} \mathrm{~F}^{\star}$ fluorescence could be measured for a given excitation of the gas by the e?ectron beam. We isolated the dimer signal using an interference filter having a bandwidth of $10 \mathrm{~nm}$. centered at $250 \mathrm{~nm}$ and the trimer signal with a Corning 5-56 color filter.'

The gas cell fill system and electron beam diode and beam transport into the cell were described in the appendix to chapter $V$. since the gas mixture generally varied from shot to shot the gases were combined in the gas cell itself. A lapse of 10 minutes between the filling of the cell and its excitation by the e-beam was sufficient to allow the gas as to mix thoroughly. In these experiments, the laser gases were excited either with the full e-beam 
plilse (at an average current density of $430 \mathrm{~A} / \mathrm{cm}^{2}$ ), or with an attenuated pulse at $160 \mathrm{~A} / \mathrm{cm}^{2}$.

\section{Laser Model}

The bulk of the kinetic equations used to model the electron beam pumped KrF laser sjstem have already been introciced in Chapters II and IV. In this section we treat the complicarions introduced by the requirement that the cavity flux arise from the amp? ification of spontaneous emission from the laser upper level, rather than being driven by an incident "seed" veam, as discussed in Chapter IV. Two results of this "bootstrapping" are the delay of the laser pulse relative to the beginning of the gas excitation and, in some cases, the absence of laser action even though the resonator shows a net positive gain.

Because of the small separation of the cavity mirrors relative to their radius of curvature $(10 \mathrm{~cm}$. vs, $1 \mathrm{~m}$.$) , the staidard resonator$ analysis found in several textbooks $s^{4,5}$ does not apply to our experimental setup. In particular, the waist of the lowest order Gaussian mode in this configuration is $<.2 \mathrm{~mm}$., much less the the transverse dimension $(\sim \mathrm{cm}$.$) of the active volume of the laser$ medium. This would imply that a large number of higher order modes should contribute significantly to the laser output. In point of fact, the laser flux fills the entire active volume, as indicated by images of the output beam on Dylux paper. In this case we have 
developed a simple model which we believe at least qua:itatively describes the buildup of the laser flux in the cavity.

As stated in the opening paragraph, we wish to model a system where the laser field builds up from the spontaneous emission from the laser upper state. It is clear, however, that only a certain fraction of the spontaneous radiation into a given solid angle will be amplified into the laser output: as those photons which are directed toward the cavity walls are absorbed by them. The time required to attain laser action in the cell depends critically on this solid angle fraction, and it is the purpose of our analysis to estimate its magnitude.

We begin our treatment of this problem by first considering the fluorescence geometry pictured in Fig. 2(a). The long, thin active region (length $\sim L$, width and height $\sim D$ ) is assumed to be populated witil a steady-state density $N$ of upper staie molecules, each of which has a spontaneous radiative lifetime $\tau_{r}$ and stimulated emission cross section $\sigma$ for photons in the frequency range of interest. We represent by $\rho(\Omega)$ the number density of photons directed in the solid angle $d \Omega$ travelling down the length of the medium. In a coordinate system moving with the photons, the rate equation determining the growth of $\rho(\Omega)$ in the absence of lass is simply

$$
\frac{d}{d t} \rho(\Omega) d \Omega=\frac{N d \Omega}{4 \pi T_{r}}+N C \sigma \rho(\Omega) d \Omega
$$


During the amplificatton of the photon denstiy to "laser" intensities, it has a negligible effect on the value of $N$, in which case $E q$. I may be solved yielding

$$
\rho(\Omega) d \Omega=\frac{d \Omega}{4 \pi c \sigma \tau_{r}}(\exp (N c \sigma t)-1)
$$

The rise of the photon density to "laser" levels is assumed to mean that it must reach a point where the stimulated decay of the upper state dominates all other decy processes, and in particular we must have

$$
N \operatorname{co} \rho(\Omega) \triangleleft \Omega \gg N / \tau_{r}
$$

Inserting Eq. 2 into Eq. 3 yields an inequality relating $d \Omega$ and $t$

$$
(\mathrm{d} \Omega / 4 \pi)(\exp (\mathrm{Nc} \sigma \mathrm{t})-1)>>1 \text {. }
$$

For the geomet, $y$ of Fig. 2(a), we see that we must have $L \geq c t$ for Eq. 4 to be applicable, in which case it implies that

$$
(\mathrm{d} \Omega / 4 \pi)(\exp (\boldsymbol{\alpha} L)-1) \gg 1
$$

where we have defined the small signal coefficient $\boldsymbol{\alpha}=\boldsymbol{\sigma} \mathbf{N}$. For a given transverse dimension $D$, there is a then a minimum length $L$ and therefore a maximum value of $\mathrm{d} \Omega\left(\mathrm{d} \Omega \simeq(0 / \mathrm{L})^{2}\right)$ for which Eq. 5 remains 
valid, which is set by the equation

$$
\frac{(\boldsymbol{\alpha} D)^{2}}{4 \pi(\boldsymbol{\alpha} L)^{2}}(\exp (\boldsymbol{\alpha} L)-1)>1
$$

Equation 6 indicates that for the geometry of Fig. $2(a)$ there is a ninimum length requirement for the system to be superfluorescent, i.?., having a power output proportional to $\mathrm{N}^{2}$ rather than $N$, a result which can be found in a more esoteric form in the literature. ${ }^{6}$ We recall $a:$ this point that we have restricted ourselves in chapter IV to consider only geometries in which the transverse gain is small, that is, with $(a D)<1$. With this limitation we find (as is illustrated in Table [), that the value of $(\boldsymbol{\alpha} L$ ) required for superfluorescence is relatively insensitive to the exact value of the transverse gain, and ranges about an average value of $(\boldsymbol{a} L) \simeq 20$.

At first glance it would seem that ine preceeding analysis has little to do with a discussion of the resonator described in Section $B$., as the values of $\boldsymbol{\alpha} L$ for the $10 \mathrm{~cm}$. cell length indicated by the results quoted in Chapter IV are typicaily $\leq 1$. Looking at Fig 2(b), however, we see that the addition of mirrors to the ends of the cell is equivalent to lengthening it, while at the same time introducing an 
effective losi coefficient

$$
\gamma=\left(2-\mathrm{R}_{1}-\mathrm{R}_{2}\right) / 2 \mathrm{~L}_{\mathrm{c}}
$$

where $R_{1}$ and $R_{2}$ are the mirror reflectivities and $L_{c}$ is the cavity length. In this case we may use the treatment above to define a minimum effective gain length $L$ for laser action in the resonator provided that we replace $\boldsymbol{\alpha}$ by

$$
\boldsymbol{\alpha}^{\prime}=\boldsymbol{\alpha}-\left(2-R_{1}-R_{2}\right) / 2 I_{C} .
$$

Once a value for $L$ is found we then have that the solid angle available for laser action is on the order of $d \Omega=(D / L)^{2}$ (where $D$ is the transverse dimension of the active region, which equals about $l \mathrm{~cm}$.$) . This factor then determines the size of the spontaneous$ emission source for the laser field.

Though the above analysis is admittedily crude (neglecting among (ther things the small curvature of the mirrors), it does explain two important effects. Since the photons must travel a distance $L$ in the gas before being amplifed to laser intensities, we see that a finite buildup time $T=L / C$ is requirad for the laser oscillation. At a "typical" value (for the argon buffered mixtures) of $\alpha=.1 \mathrm{~cm}^{-1}$, and witn $R_{1} \simeq 1, R_{2}=.5$, we would have $\alpha^{\prime}=.075 \mathrm{~cm}^{-1}, L=270 \mathrm{~cm}$. and $T \simeq 9$ ns., comparable to but less than the excitation pulse width of about $50 \mathrm{~ns}$. For cases where $\boldsymbol{\alpha}$ is smaller, the buildup will take 
even longer, and for extreme cases laser action may not occur either due iu a negative value for $\boldsymbol{\alpha}^{\prime}$, or due to the fact the the rise time $T$ is significantly greater than the duration of the e-beam pulse. It is in fact for these very reasons that the $70 \%$ reflective mirrors had to be used to achieve laser action in the helium buffered mixtures with their smaller gain coefficients.

The end result of the above discussion is that the rise of the laser flux was modelled in the following manner. For a given gas mixture and eiectron beam current density the code CELLKIN described in Chapter IV was first run to determine the small signal gain coefficient which would appear in the cell in the absence of laser action. The solid angle a $\Omega$ tor the laser flux was then calculated using the mirror reflectivities of interest, and a fraction $d \Omega / 4 \pi$ of thz sprntaneous emission was assumed to serve as the source of the laser signal in each direction. This ratio was then used as input to the computer program LASRES ( 1 isted in the microfiche at the end of this thesis), which is similar to CELLKIN but which tracks the full laser kinetics according to the methods described above in Chapter IV and in the above discussion. With regard to the photon transport, a single zone was found sufficient for the modelling, since in all cases $\boldsymbol{\alpha} L \leq 1$. The results of these calculations are to be found in the following section. 


\section{Results of Experiment and Calculation}

\section{(i) Introduction}

We begin this section by noting that the experimental setup described in Section B, allows the variation of a large number of the quantities (e. g., the current density, mirror reflectivities, buffer gas, fluorine donor, etc.) which determine the laser output. A detailed study carried out over the entire relevant range of this multi-dimensional parameter space is well beyond the scope of this thesis. Rather, to reduce the problem to a tractible size it was necessary to fix most of these variables at somewhat arbitrary values, and investigate the functional dependence of the laser performance only over the remaining few.

Referring specifically to the results presented below, we applied the following restrictions:

Average current density: either 100 or $430 \mathrm{~A} / \mathrm{cm}^{2}$;

Fluorine donor: molecular fluorine $\left(F_{2}\right)$;

Buffer gas: either 1500 Torr of argon or

11,600 Torr of heliuin;

Mirror reflectivities: maximum reflectivity (99\%) and 50\% (70\%) reflective mirrors with argon (helium! as the buffer gas.

The values of the current density, choice of fluorine donor, and argon buffer gas pressure selected were typical of what is required 
for $\mathrm{KrF}$ laser operation, The helium buffer pressure was selected to yield the same energy aeposition from the $600 \mathrm{keV}$ electron beam as would occur in the argon mixture, according to tabulated stopping powers. ${ }^{7}$ Finally, the output mirror for the laser cavity was chosen merely by the requirement that its relectivity was sufficient to achieve experimentally observable laser action at some time during the 50 ns. e-beam puise.

Under the limitations described above, the laser efficiency (defined as the ratio of the output energy to the energy deposited by the e-beam in the laser medium) was experimentally optimized as a function of both the $F_{2}$ and krypton gas concentrations for both buffer gases at both current densities. In each case we first determined the optimum $F_{2}$ pressure at an arbitrary krypton pressure of 100 Torr; using this fluorine concentration the kryton density yielding the maximum laser efficiency was then sought out. Finally, the $F_{2}$ density was then varied to insure that at least a local optimum had been found in this two variable parameter space. In all cases the energy deposited in the laser gas was assumed to equal twice that for a single pass of the $600 \mathrm{keV}$ electron beam, as was previously discussed in Section D. of Chapter IV.

Upon completion of the experimental work the KrF laser kinetics model described in Chapters II and IV, and in Section Co of this chapter, was used to predict the laser aup 1 t from the gas mixtures and 
laser configurations investigated experimentally. The comparison of the results of the model calculations with those observed experimentally is described in detail in subsections (ii) and (iii) below. In general the agreement between the model and experinient was better for the argon buffered mixtures than for those with helium as the bufier gas, and also for the hore intensely pumped samples at $430 \mathrm{~A} / \mathrm{cm}^{2}$ as compared to those at $160 \mathrm{~A} / \mathrm{cm}^{2}$. Possible reasons for this behavior will be discussed in Section $E$.

\section{(ii) Argon Buffered Mixtures}

The experimental optimization of the laser efficiency in argon buffered mixtures at both current densities is depicted graph, aily in Figs. $3-6$, and the optimal gas pressures and maximum efficiencies are summarized in the first part of Table II. In Figs. 3 and 4 it is sufficient to show only the laser output energy, as the variation of the deposited energy with $F_{2}$ pressure was negligible. When the krypton pressure was varied this was obviously not the case as is apparent in Fig. 5 where the deposited energy changed by more than a factor of 2 over the range in krypton concentration.

The qualitative dependence of the laser output on the fluorine pressure exhibited in Figs, 3 and 4 is easily expressed as a competition between several processes. First, at the lower fluorine concentrations the KrF production rate suffers from a decrease in the density of its fluorine precursors $\left(F^{-}\right.$and $\left.F_{2}\right)$, and the density of thermal electrons in the medium rises (since the primary loss process 
for these electrons is dissociative attachment to $F_{2}$ ) causing an increase in the KrF dimer destruction rate. Both of these processes result in a fall-off in the output energy as the $F_{2}$ pressure is decreased; at the lawest fluorine concentrations both are magnified by the depletion of the initizi $r_{2}$ density, causing a rapid decline in the laser output. Conversely, as the fluorine pressure is increased the surfeit of molecular $F_{2}$ results in an increased heavy particle quenching rate for the dimer, eventually causing a decrease in the output energy at the highest fluorine concentrations

The dependence of the laser output on the krypton pressure illustrated in Figs. 5 and 6 is the result of a similar competition. At the lowest krypton pressures the $\mathrm{KrF}$ production rate rises with the krypton concentration while its destruction rate remains essentially constant. At elevated krypton densities the heavy particle two- and three-body quenching of the laser upper state begins to rise faster than its creation rate, causing an eventual decrease in the laser signal.

The prediction of our laser kinetics model for the laser output energies is presented along with the experimental data in Figs. 3 - 5. In all of these calculations it was assuned that the energy deposition rate from the e-beam into the gas was equal to twice the value from a single pass as discussed above. The agreement between the expermental and calculated outputs at $430 \mathrm{~A} / \mathrm{cm}^{2}$ is quite 
good with regard to both the absolute magnitude and shape of the two curves. In Fig. 4 at $160 \mathrm{~A} / \mathrm{cm}^{2}$ the result of experiment and calculation are qualitatively similar, but there is a definite shift in the position and magnitude of the peak signal between the two curves.

The absence of a plot of the predicted laser output for the experimental conditions of Fig. 6 is due to the fact that over most of the relevant range of krypton pressures (i.e., values significantly different from 100 Torr) the predicted output energy was essentially zero. As will be discussed more completely in Section E., we believe that the radical difference between the results of experiment and the model stems from an inherent inacuracy in the application of the laser build-up approximation of Section $C$. in the case of laser operation near threshold.

This variation in the agreement between model and experiment is also evident in the time dependence of the laser output as illustrated in Figs. 7 and 8 . The experimental output power in both cases was calculated by normalizing the time dependence of the signal measured by the photodiode through the maximum reflectivity mirror to the total energy exitting the output mirror. The degradation in the qualitative fit of the model to the experiment as the current density decreased is easily seen. While there is good agreement at $430 \mathrm{~A} / \mathrm{cm}^{2}$, at the lower value of $160 \mathrm{~A} / \mathrm{cm}^{2}$ the magnitudes, shapes, and durations of the two curves all are different. 


\section{(iii) Helium Buffered Mixtures}

Data from the experimental optimization of the laser efficiency with the krypton and fluorine gas pressures with helium as the buffer gas are presented in Figs. $9-12$, and the final results are summarized in the second half of Table II. The magnitude of the output signal varied in the same qualitative manner as was seen above for the argon buffer mixtures, but the peak efficiencies were found to be lower at both valijes of the current density. This latter result has two apparent causes: the decreased energy efficiency for creating the $\mathrm{KrF}$ upeer state with a helium buffer (due to the higher values for the energies of the excited and ionic stites of 1. ?lium as compared to this of argan), and the increased que.lching of the $\mathrm{KrF}$ dimer present at the elevated helium pressur:s required to obtain the same energy deposition as in the lower pressure argon mixtures.

Due to the relative uncert. "nty in the kinetics of helium buffered KrF laser media (as compared to the wealth of data available for the argon buffered kinetics) we decided to aiso fireasure the $\mathrm{KrF}$ and $\mathrm{Kr}_{2} \mathrm{~F}$ sidelight fluorescence signals from the gus cell with the laser mirrors removed for the asje of yarying fluorine pressure as a test of our understanding of the rocesses involved. The results of these calculations are presented in Figs. 9 and 12 along with the corresponding laser oucput signals. At both values of the current density the dimer fluorescence signal and laser output snergy peak at the same value of the fluorine concentration, as we would expect in the situation where the medium gain cominates the loss, and as is 
predicted by the kinetics model. The uccurance of the peak of the trimer signal at a lower value of the fluorine pressure than that of dimer is explained by the fact that neavy particle quenching by $F_{2}$ is more important for $\mathrm{Kr}_{2} \mathrm{~F}$ as a result of its much longer radiative lifet ime.

The predictions of the laser kinetics model for the laser output from mixtures pumped at $430 \mathrm{~A} / \mathrm{cm}^{2}$ are presented in Figs. 10 and 11 . In order to obtain any agreement with the experimental results it was necessary to include in the kinetics a two-body quenching reaction for the KrF upper state by helium, with a rate constant of $2 \times 10^{-12} \mathrm{~cm}^{3} \mathrm{~s}^{-1}$. Because of the large helium concentrations required to achieve is significant deposition from the electron beam we would expect two-body quenching to be a much more important effect here than in the argon buffered mixtures. That this reaction proceeds at such a rate seen.s likely, in line with the measured reaction coefficient of $7.4 \times 15^{-13} \mathrm{~cm}^{3} \mathrm{~s}^{-1}$ for the destruction of $\mathrm{KrF}$ by krypton quoted in Cliapter IX.

It is important to note at this point that reaction rate constants which require a full kinetic model calculation for their evaluation, as was the case for the two-body quenching coefficient above, should in general be held as suspect. It is quite possible that the disagreement between the model and experiment is the result of some other inaccuracy in the kinetic mode?. The rate constants used in the model for the various reactions should as a rule be the 
product of experiments which unequivocally focus on on the relevant subset of the full kinetics.

With this caveat in mind we see that while the predictions of the model at $430 \mathrm{~A} / \mathrm{cm}^{2}$ do not differ radiacally from the experimental values, the agreement between the two is obviously worse than was seen for the argon buffered mixtures of subsection ( $i i$ ). This point is also evident in the match between the proficted and observed time dependence of the laser output, plotted in Fig. 13. The curve for the experimental output power was obtained by normalizing the corresponding photodiode signal, as discussed above. Though the total output energy was approximately the sume for the two cases, the experimental signal was of longer duratior: and lower magnitude than predicted by the calculation.

At the lower value of the current density $\left(160 \mathrm{~A} / \mathrm{cm}^{2}\right)$ the problems with the model calculation mentioned above for the argon buffered mixtures were even more apparent. The calculated output energy was not appreciably different from zero for any of the mixtures considered. Here we feel that this is due both to inaccuracies in the kinetics model (which are more likely here because of the paucity of da:a for the helium ouffered kinetics), and to the inapplicability of our laser build-up approximation in the region close to threshald. Because of the lower efficiencies for the helium buffered mixtures as compared to those using argon we would expect this latter problem to 
be more important in the present zase. Both of these difficulties will be discussed more thoroughly in the following section

\section{E. Discussion}

As evidenced by the comparisons of the experimental data and model predictions for KrF laser operation presentea in Section D., the primary factor determining the quality of the fit between the two appeared to be the amount of gas excitation. At the larger value of the current density $\left(430 \mathrm{~A} / \mathrm{cm}^{2}\right)$ the fit was quite good for all the data from the argon buffered media and there was agreement between the predicted and observed output energies for the mixtures where helium was the buffer. At $160 \mathrm{~A} / \mathrm{cm}^{2}$, however, the calculated values for the laser output differed quite radically from those actually observed, effectively equaling zero for most of the cases corsidered.

We believe that this failume of the kinetics model at the lower current density was actually due to an inaccuracy involved in using the laser flux build-up approximation of Section $C$. in the threshold regime (i.e., in situations where the intensity in the laser medium was always less than the saturation intensity). In the "boot-strap" model above we stated that laser operation was achieved when the photon density reached a point where stimulated Enission by the laser upper level dominated all its other decay processes. We then estimated the angle $d \Omega$ inside which the spontaneous emission from the dimer could be amplified to such levels before hitting one of the 
cavity walls. Finally, we assumed that only the radiation directed inside this range had to be considered in determining the laser output through the cavity mirrors. In situations where the net effective gain (including the mirror losses) was large enough for the radiation in the cone $d \Omega$ to grow to "laser" levels in a time short compared to the duration of the excitation pulse such an approach was valid because this "mode" rapidly saturated the laser transition and prevented the intensity directed in other directions from becoming appreciable. When the time required for the laser buildup exceeded the e-beam pulse width, however, no such domination of the KrF* decay occurred and the flux into other solid angles could reach levels comparable to that directed in the limited range $d \Omega$.

A short calculation illustrates the magnitude of this effect fer our experimental setup as the gain in the cell decreased. Due to the 50 ns. length of the pump pulse the maximum small signal gain achievable by the radiation in the gas cell at an effective gain coefficient of $.003 \mathrm{~cm}^{-1}$ was $\simeq 4.5$, and the solid angle inside which the spontaneous emission could experience this growth without striking the walls was about $1.5 \times 10^{-6}$ sterad. In contrast, for the same parameters the solid angle for laser operation calculated according to Ey. 6 is $5 \times 10^{-8}$ sterad., indicating that the buildup model above underestimated the energy output by at least a factor of 25 .

We see that any method which is meant to accurately model the photon transport in the laser medium at low levels of excitation must 
take into account the angular dependence of the amplified radiation, which is to say that it must irivolve at least a two-dimensional (spatial) calculation. Such a treatment is also required for any configuration for which the gain in directions other than the laser axis is appreciable. In this latter case amplified stimulated emission uan present a significant loss mechanism for the KrF upper state and in addition cause a transverse variation in the gain profile.

Since such multi-dimensional calculations can te accomplished only at greatly increased calculational cost it is valuable to note that the one-dimensional mocel above is valid for those configurations where the distribution of amplified radiation is dominated by a single "mode". One situation where this assumption applies has been demonstrated above in the behavior of our laser resonator at high values of the effective gain coefficient. We would also expect our approximation to accurately treat the case of a single pass amplifier which is probed at an input intensity greater than the saturation intensity of the medium.

Limiting ourselves to the consideration of only those cases where our one-dimensional treatment of the radiation vas valid, we have found that for argor, buffered mixtures of krypton and fluorine the $\mathrm{krF}$ :aser kinetics model accurately predicted the experimental results over a wide range of excitation levels and gas pressures. For the mixtures where helium was used as the buffer, the agreement between the model anti experiment was somewhat less clear. Al though the 
calculated magnitudes for the laser output were of the same approximate size as those observed experimentally, their variation with the gas pressures and time dependence leads us to suspect that there is room for improvement in our treatment of the helium buffered kinetics.

To see how this might be accomplished we refer back to Table II of Chapter IV, where we first note that for want of better values the rate constants for several of the reactions involving the transfer of excitation from helium to krypton were set equal to those for the corresponding reactions involving argon. The proper values of these rate coefficients could be determined simply by repeating the procedures by which the argon - helium data was obtained, 8-10 substituting krypion for the argon gas component. A similar lack of experimental data forced us to assume that all of the reactions involving the excited states of fluorine proceeded at their gas kinetic values, an approximration which was justified by the fact that a negligible fraction of the gas excitation $(<i \%)$ typically passed through these channels. Nevertheless, this situation could be improved by an experiment analogous to the one in which piestis et al. studied the quenching of $F^{\star}$ and $F_{2}{ }^{\star}$ in e-beam excited mixtures of neon, xenon, and $F_{2},{ }^{11}$ again making the appropriate gas substitutions. Finally, with regard to the value for the rate coefficient for the two-body quenching of $\mathrm{krF}$ by nelium postulated in Section D., it should be possible to experimentally measure this quallity via a technique similar to that used by Rokni et. al. to 
determine the destruction coefficients for the dimer in e-beam excited $\mathrm{Ar} / \mathrm{Kr} / \mathrm{F}_{2}$ gas mixes. 12

We conclude by noting that in the introduction to this thes is we stated that it was our purpose to "develop a kinetic model for the KrF* laser system", and specifically for "electron beam pumped $\mathrm{Kr}-\mathrm{F}_{2}$ mixtures, buffered with either helium or argon." Computational complexity has forced us to consider only a limited set of laser configurations for which the distribution of amplified radiation is dominated by a single "mode". Within this restriction we believe we have fulfilled our or:ginal intentions with regard to the description of systems typical of KrF laser operation, though further future improvement in the model regarding helium kinetics is possible and awaits further experimentation. 
Table I

Small Signal Gain Required for Superfluorescence

\begin{tabular}{cc}
$\begin{array}{c}\text { Transverse Small } \\
\text { Signal Gain } \\
(\boldsymbol{\alpha} \alpha)\end{array}$ & $\begin{array}{c}\text { Axial Gain } \\
\text { Required } \\
(\boldsymbol{\alpha} \mathrm{L})\end{array}$ \\
\hline .003 & - \\
.01 & 22.7 \\
.03 & 20.0 \\
.7 & 17.6 \\
& 14.8
\end{tabular}

(Note: The minimum required gain was found by setting the left-hand side of equation (6) equal to 10.) 
Table II

Result of the Experimental optimization of the Laser Efficiency at Fixed Buffer Gas Pressure and Current Density

$$
\text { Buffer Gas: } 1500 \text { Torr Argon }
$$

Average Current

Density
$\frac{\left(\mathrm{A} / \mathrm{cm}^{2}\right)}{160}$

430

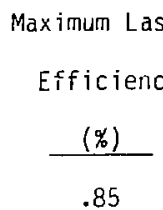

4.2
Krypton

Pressure

(Torr)

100

300
$\mathrm{F}_{2}$

Pressure (Torr)

2.5

6.0

Buffer Gas: 11,600 Torr Helium

Average Current

Density

$\left(\mathrm{A} / \mathrm{cm}^{2}\right)$

160

430
Maximum Laser

Efficiency

$(\%)$

.17

1.3
Krypton

Pressure

(Torr)

50

200
$\mathrm{F}_{2}$

Pressure (Torr)

4.0

5.0 


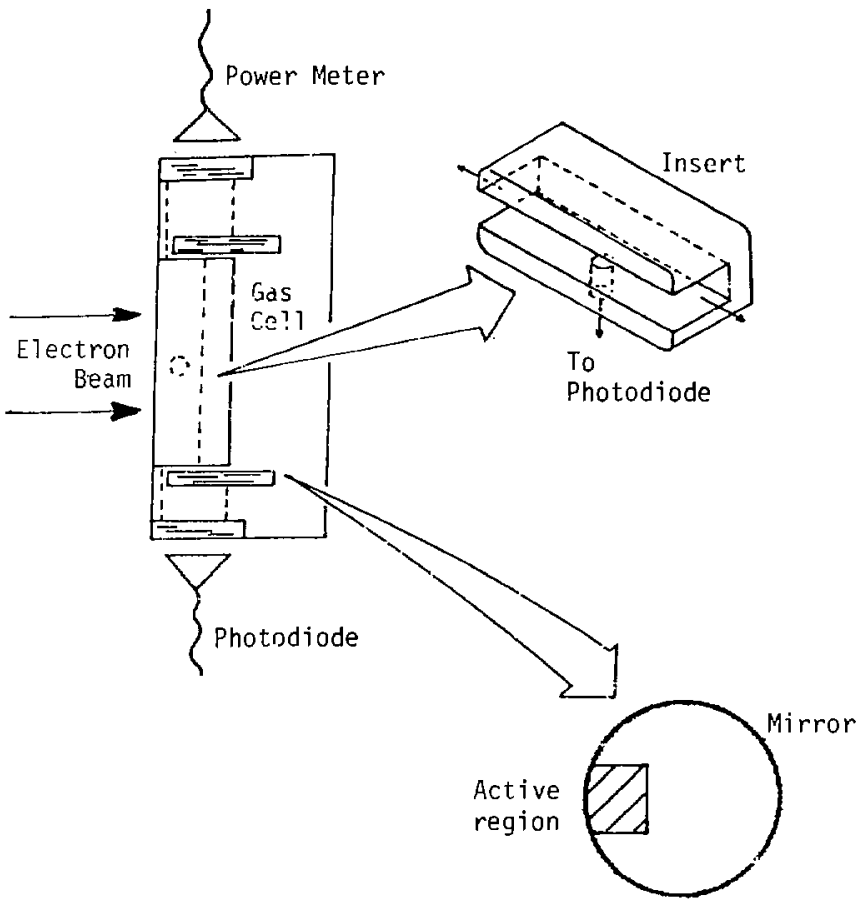

Fig. 1. Schematic illustration of the modifications to the MEG I gas cell used to produce and observe $\mathrm{krF}$ laser action. 
(a)

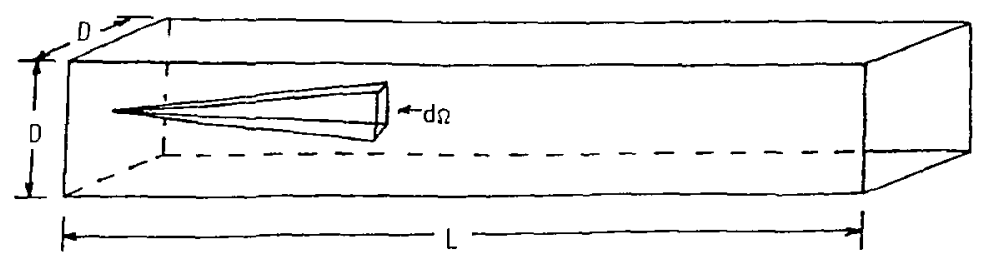

(b)
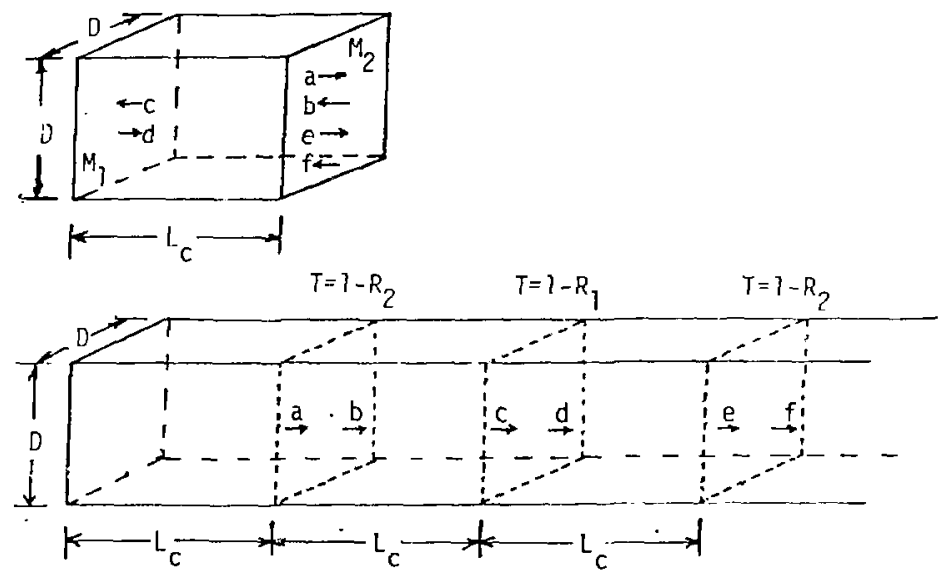

Fig. 2. (a) Geometry for the superfluorescence calculation of Section $B$.

(b) Demonstration of the "unfolding" of the laser cavity to resemble the superfluorescent geometry. The mirror reflectivities are $R_{1}$ and $R_{2}$, respectively.

(N.B. In both (a) and (b) the transverse dimension (D) is greatiy exagerated) 


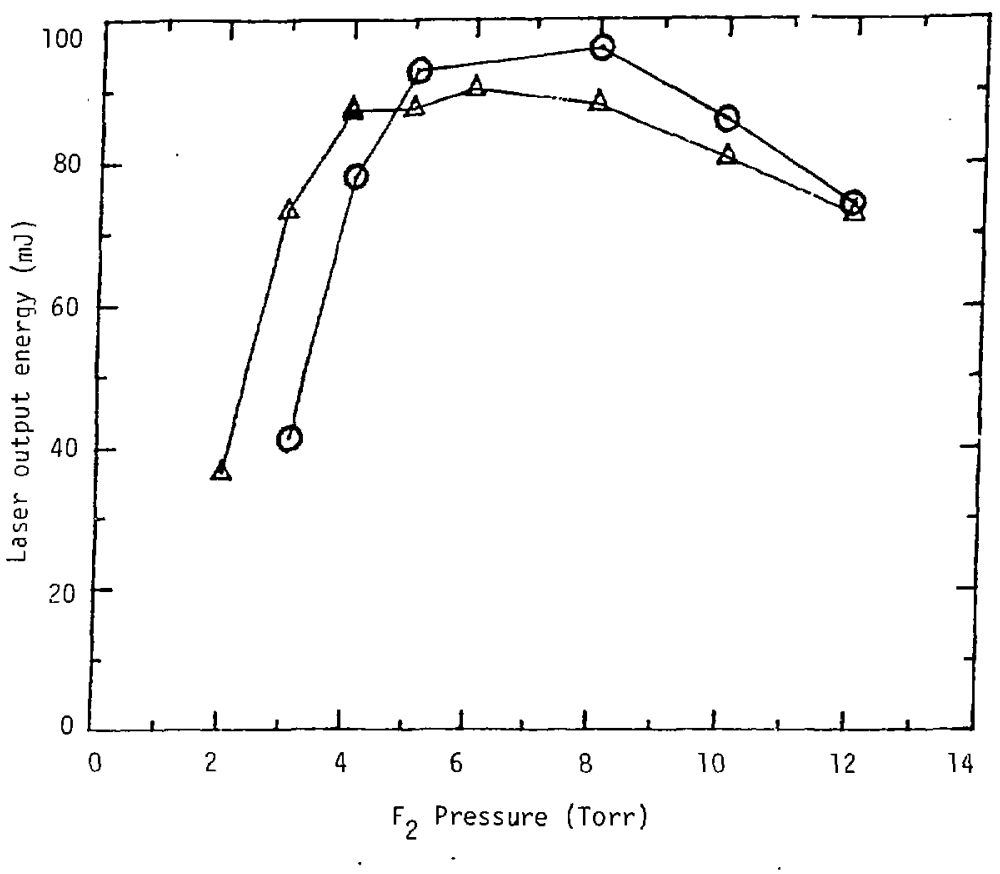

Fig. 3. Laser output energy vs $F_{2}$ pressure from 1500 Torr Ar - 100 Torr $\mathrm{Kr}-F_{2}$ gas mixtures at an average current density of $430 \mathrm{~A} / \mathrm{cm}^{2}:(\triangle)$ experimental; (O) calculated. 


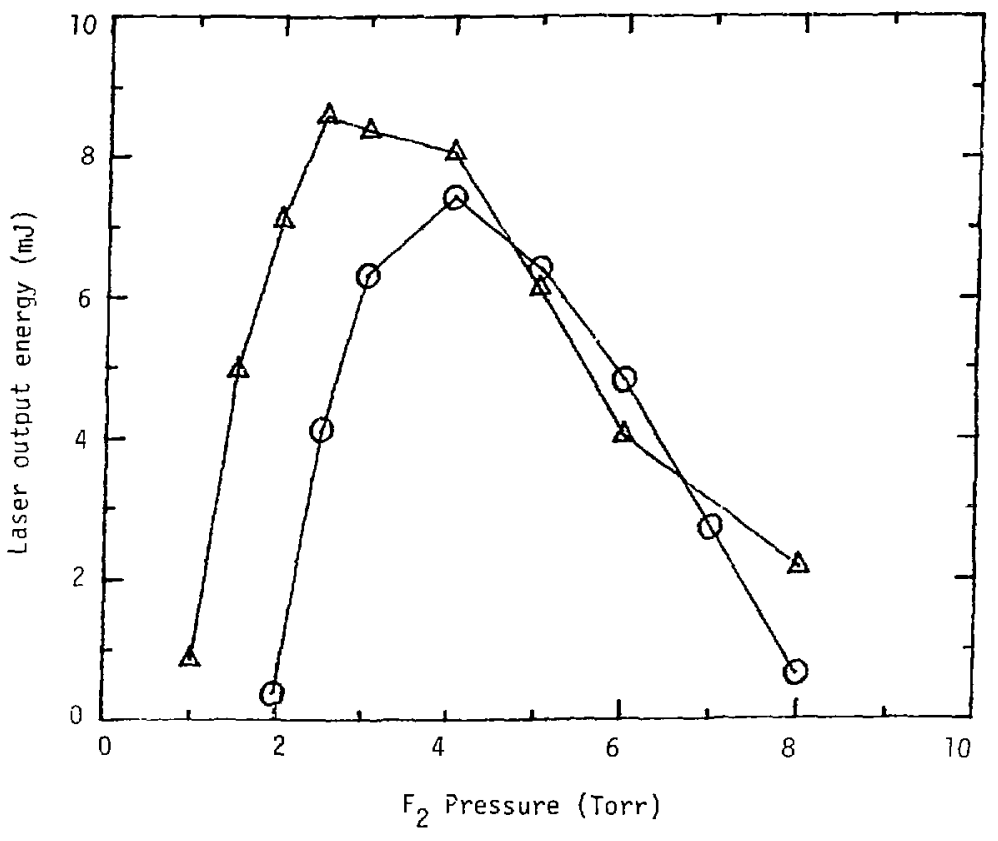

Fig. 4. Laser output energy vs $F_{2}$ pressure from 1500 Torr Ar - 100 Torr $\mathrm{Kr}-\mathrm{F}_{2}$ gas mixtures at an average current density of $160 \mathrm{~A} / \mathrm{cm}^{2}$ :

$(\triangle)$ experimental; (O) calculated. 


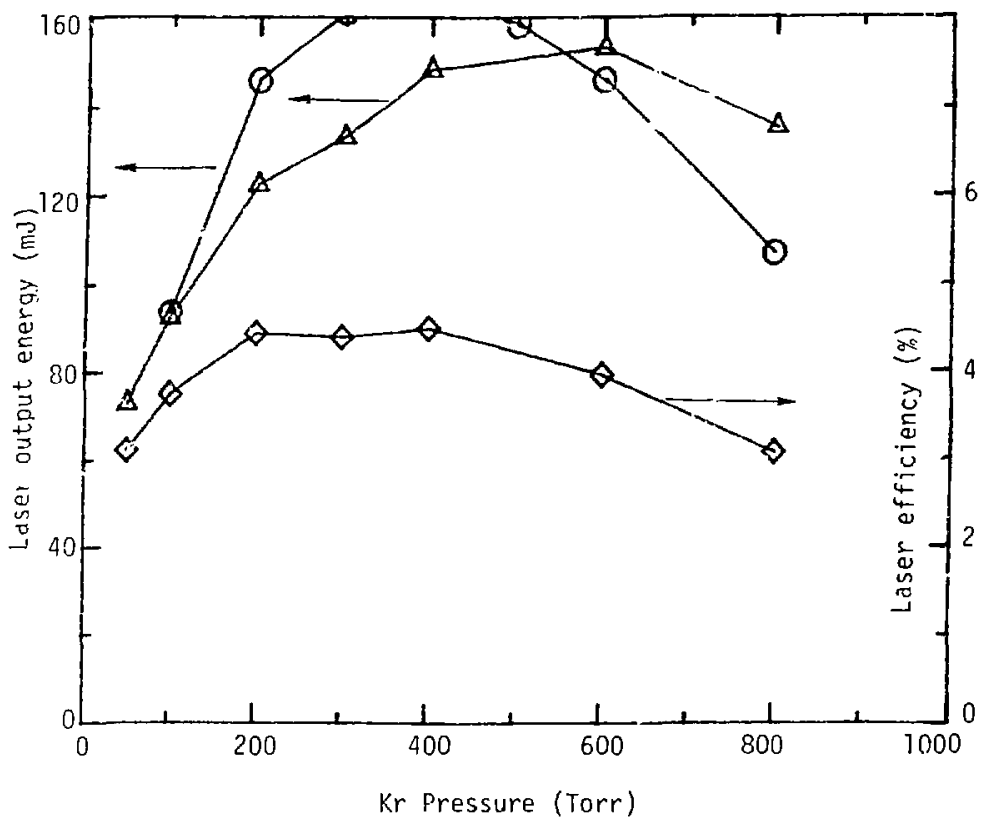

Fig. 5. Laser output. energy and laser efficielıcy vs $\mathrm{Kr}$ pressure from 1500 Torr Ar - 6.00 Torr $\mathrm{F}_{2}-\mathrm{Kr}$ gas mixtures at an average curreni density of $430 \mathrm{~A} / \mathrm{cm}^{2}$ : ( $\Delta)$ experimental; (O) calculated; $(>)$ experimental. (At 400 Torr $\mathrm{Kr}$ the calculated value is $165 \mathrm{~mJ}$ ) 


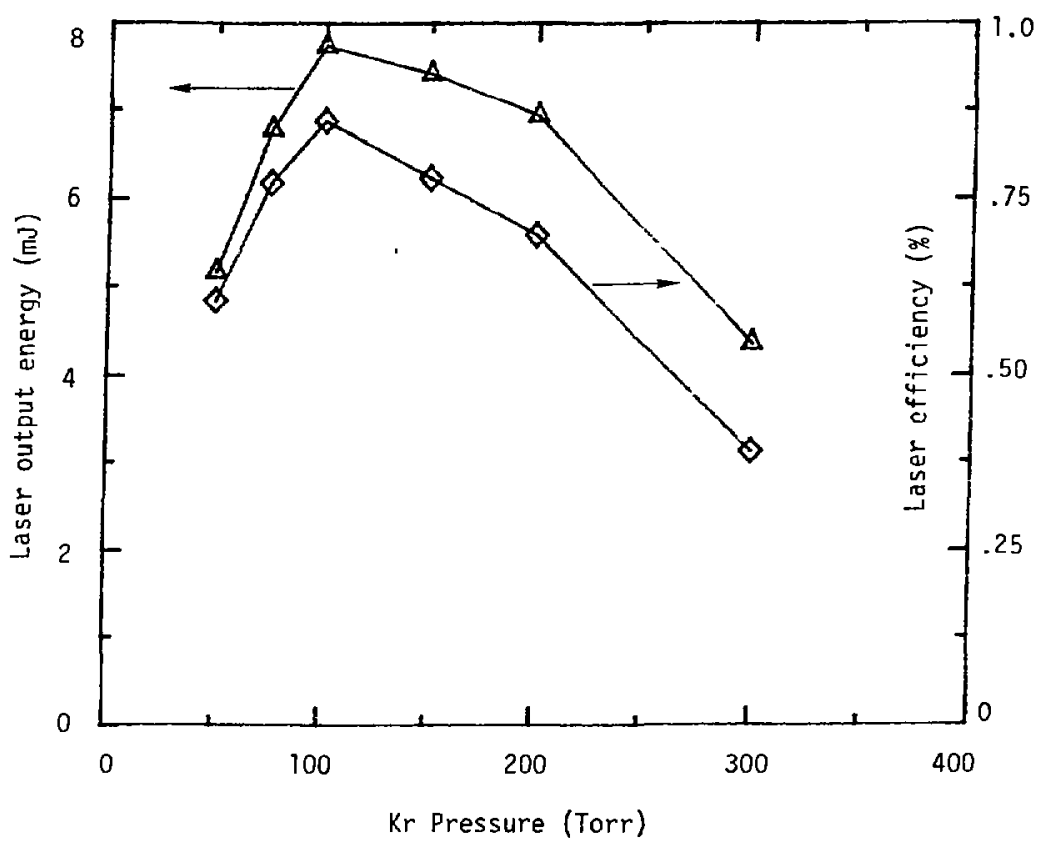

Fig. 6. Coserved laser output energy and laser efficiency vs $\mathrm{Kr}$. pressure from 1500 Torr Ar - 2.50 Torr $\mathrm{F}_{2}-\mathrm{Kr}$ gas mixtures at an average current density of $160 \mathrm{~A} / \mathrm{cm}^{2}$. 


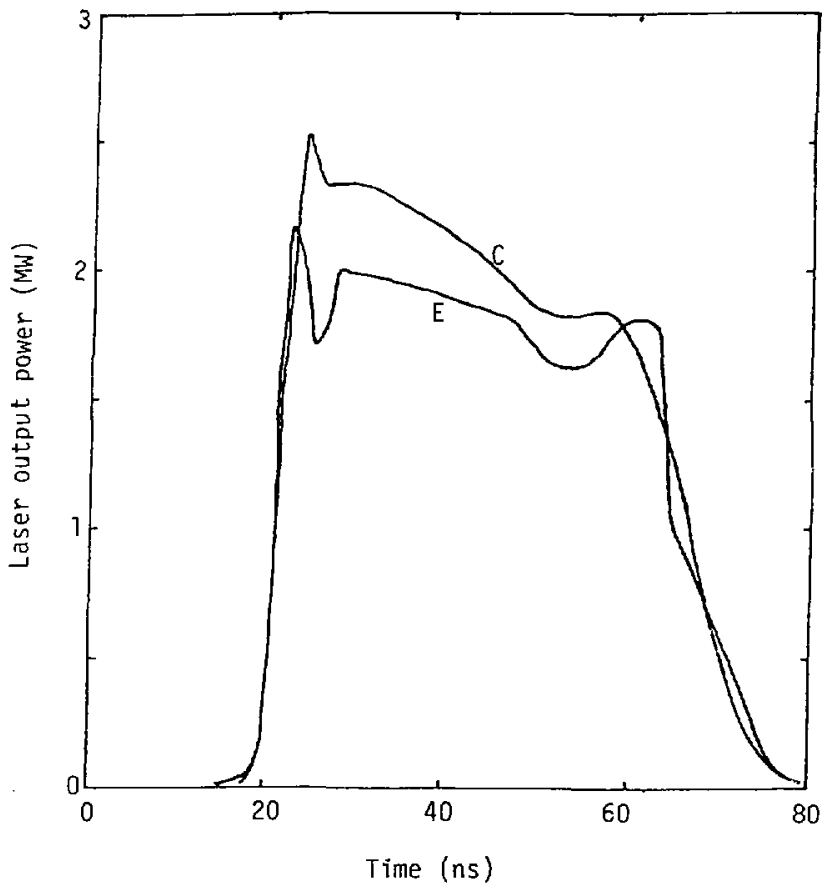

Fig. 7. Time dependence of the laser output from a 1500 Torr Ar - 100 Torr $\mathrm{Kr}$ - 5.00 Torr $F_{2}$ gas mixture pumped at an average current density of $430 \mathrm{~A} / \mathrm{cm}^{2}:$ (E) experimental; (C) calculated. 
212

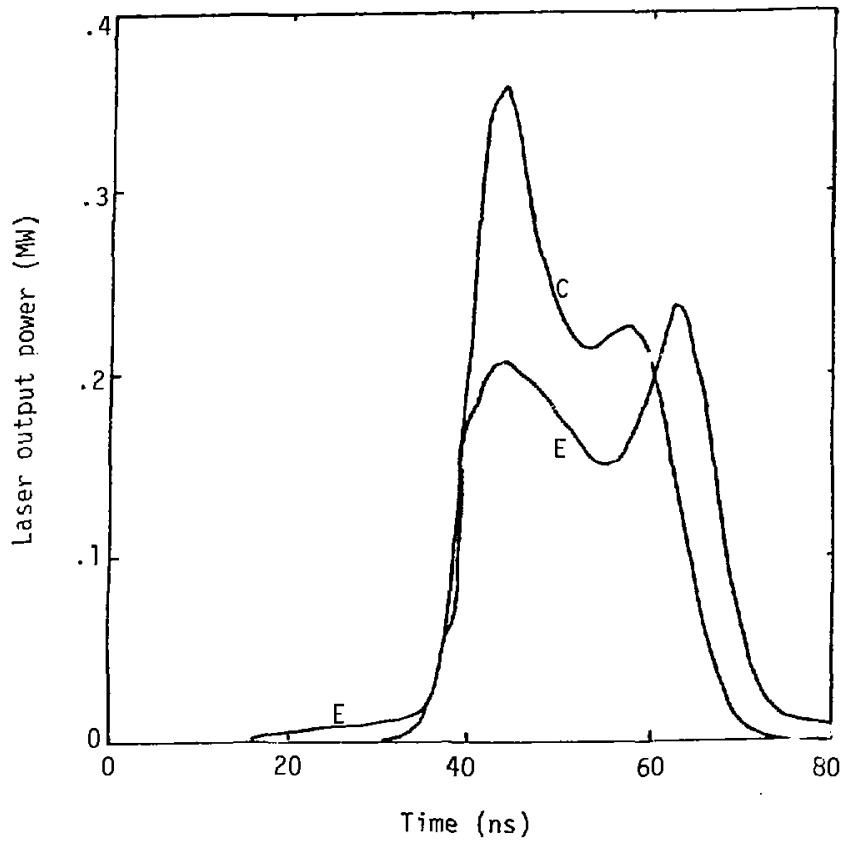

Fig. 8. Time dependence of the laser output from a 1500 Torr Ar - 100 Torr $k r$ - 5.00 Torr $F_{2}$ gas mixture pumped at an average current density of $160 \mathrm{~A} / \mathrm{cm}^{2}$ : (E) experimental 1; (C) calculated. 


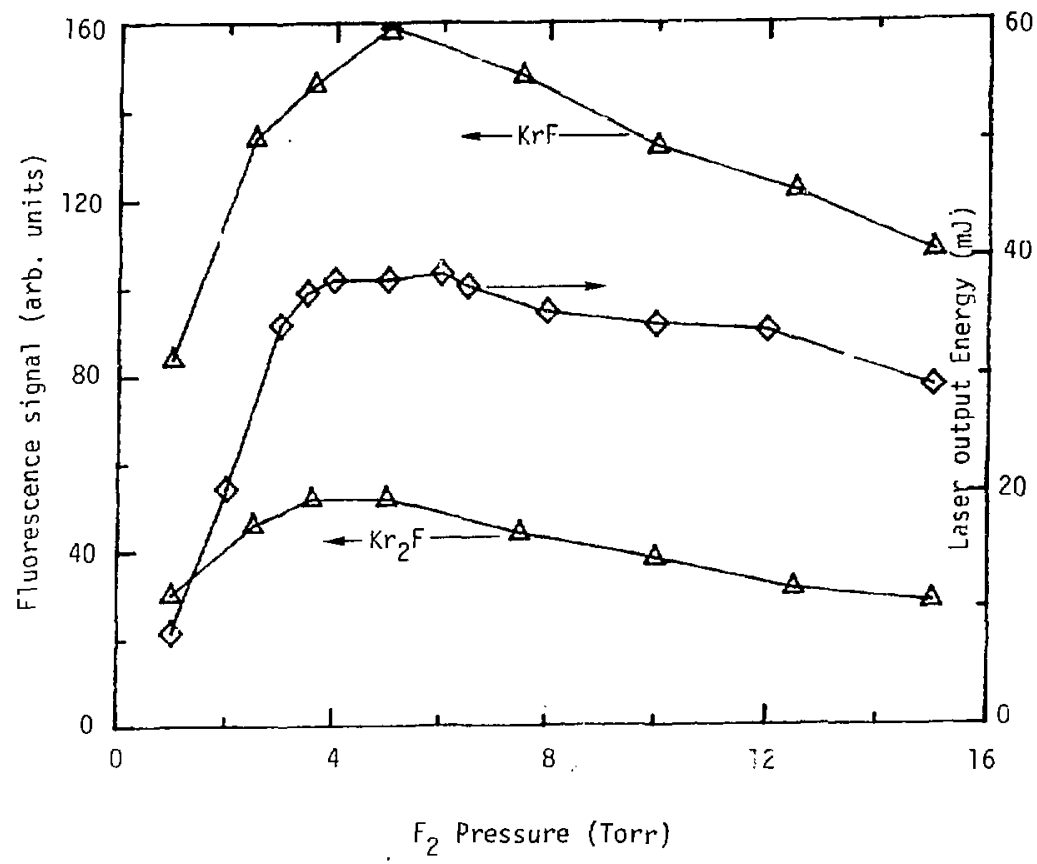

Fig. 9. Observed f7uorescence signa) (no mirrors) and laser output energy (with mirrors) vs $F_{2}$ pressure from 11,600 Torr He - 100 Torr $\mathrm{Kr}-\mathrm{F}_{2}$ gas inixtures at an average current density of $430 \mathrm{~A} / \mathrm{cm}^{2}$. 


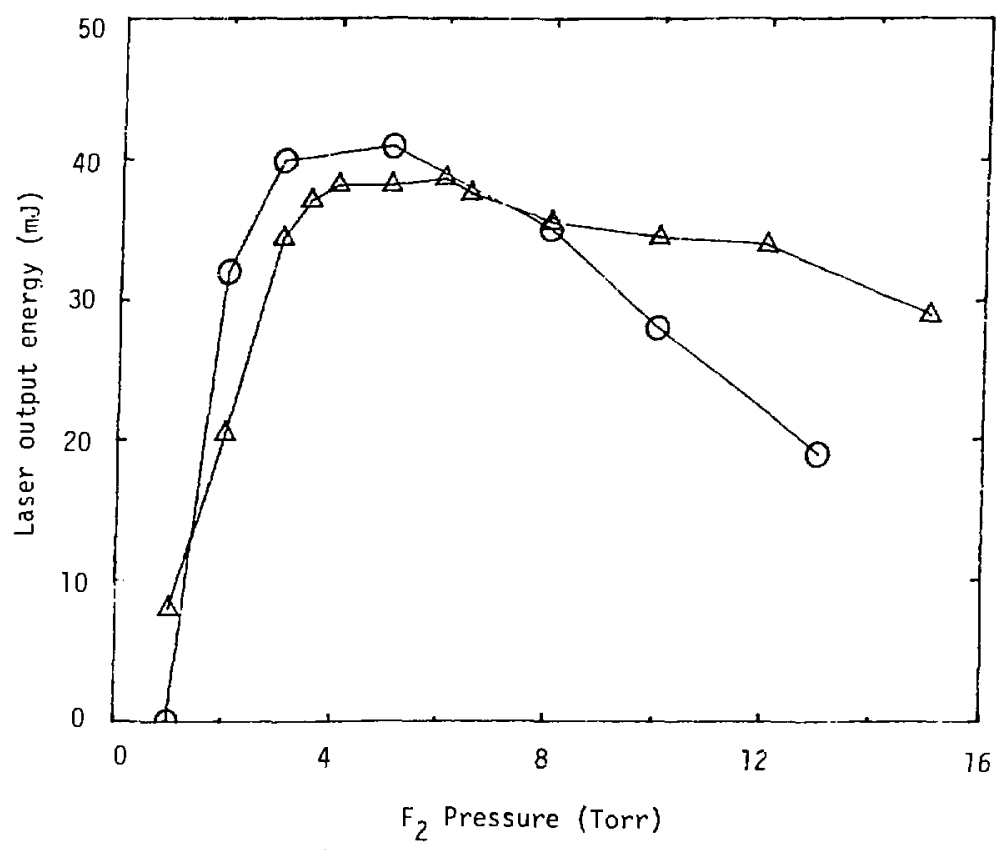

Fig. 10. Laser output energy vs $F_{2}$ pressure from 11,600 Torr He - 100 Torr $\mathrm{Kr}-\mathrm{F}_{2}$ gas mixtures at an average current density of $430 \mathrm{~A} / \mathrm{cm}^{2}$ : ( $\Delta$ ) experimentai; $(O)$ calculated. 


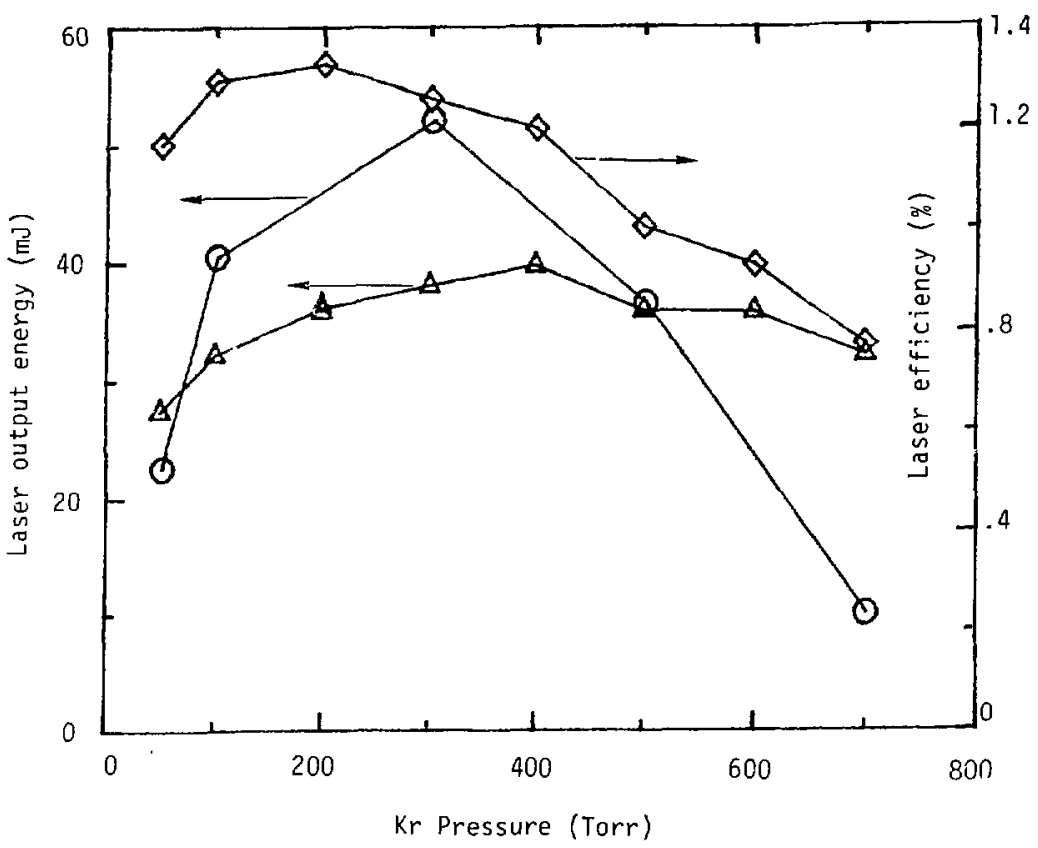

Fig. 11. Laser output energy and laser efficiency vs $\mathrm{Kr}$ pressure from 11,600 Torr He - 5.00 Torr $\mathrm{F}_{2}-\mathrm{kr}$ gas mixtures at an average current density of $430 \mathrm{~A} / \mathrm{cm}^{2}$ : ( $\triangle$ ) experimental; (O) calculated; $(\supset)$ experimental. 


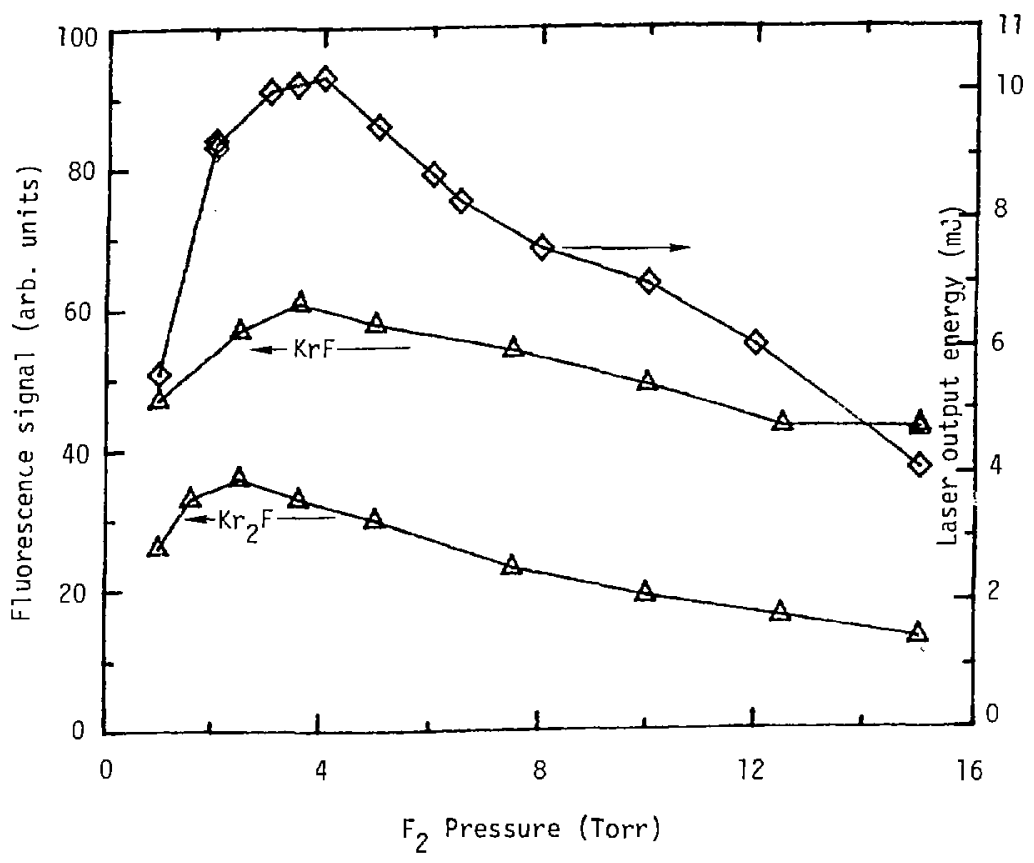

Fig. 12. Observed fluorescence signals (no mirrors) and laser output energy (with mirrors) vs $F_{2}$ pressure from 11,600 Torr $\mathrm{He}$ - 100 Torr $\mathrm{Kr}-\mathrm{F}_{2}$ gas mixtures at an average current density of $160 \mathrm{~A} / \mathrm{cm}^{2}$. 


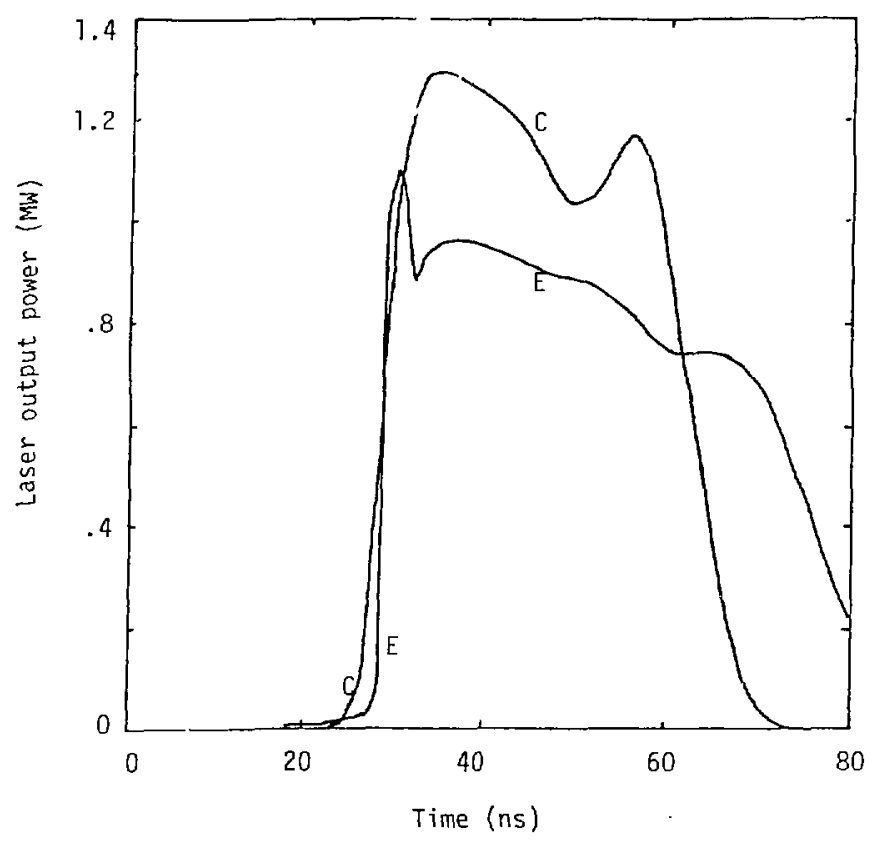

Fig. 13. Time dependence of the laser output from a 11,600 Torr He - 100 Torr $\mathrm{Kr}-5.00$ Torr $F_{2}$ gas mixture pumped at an average current density of $430 \mathrm{~A} / \mathrm{cm}^{2}$ : (E) experimental; (C) calculated. 
References - Chapter VI

1. Reference to a company or product name does not imply approval or recommendation of the product by the University of California or the U.S. Department of Energy to the exclusion of others that may be suitable.

2. S. R. Gunn, UCID-16608, Lawrence Livermore Laboratory (1974)

3. M. W. Taylor, J. Goldhar, and J. R. Murray, Appl. Opt. 21, 4 (1982)

4. A. Yariv, Quantum Electronics, $2^{\text {nd }}$ Ed., John Wilcy \& Sons, New York (1975)

5. A. E. Siegman, Introduction to Lasers and Masers, McGraw Hill, New York (1971)

6. R. Bonafacio and L. A. Luqiato, Phys. Rev. All, 1507 (1975)

7. L. Pages, E. Bertel, H. Joffre, and L. Sklavenitis, Atom. Data 4 , 1 (1972)

8. F. W. Lee, C. B. Collins, and R. A. Waller, Jrnl. Chem. Phys. sis, $1605(1976)$

9. F. W. Lee, arid C. B. Collins, Jrnl. Chem. Phys. 65, 5189 (1976)

10. F. W. Lee, and C. B. Collins, Jrnl. Chem. Phys. 67, 2798 (1977)

11. D. L. Huestis, R. M. Hill, H. H. Nakano, and D. C. Lorents, Jr!ll. Chem. Phys. 69, 5133 (1978)

12. M. Rokni, J. H. Jacob, and J. A. Mangano, Phys. Rev. Al6, 2216 (1977) 
Microfiche Listings of Computer Programs 